\title{
Analysis and evaluation of BSDF characterization of daylighting systems
}
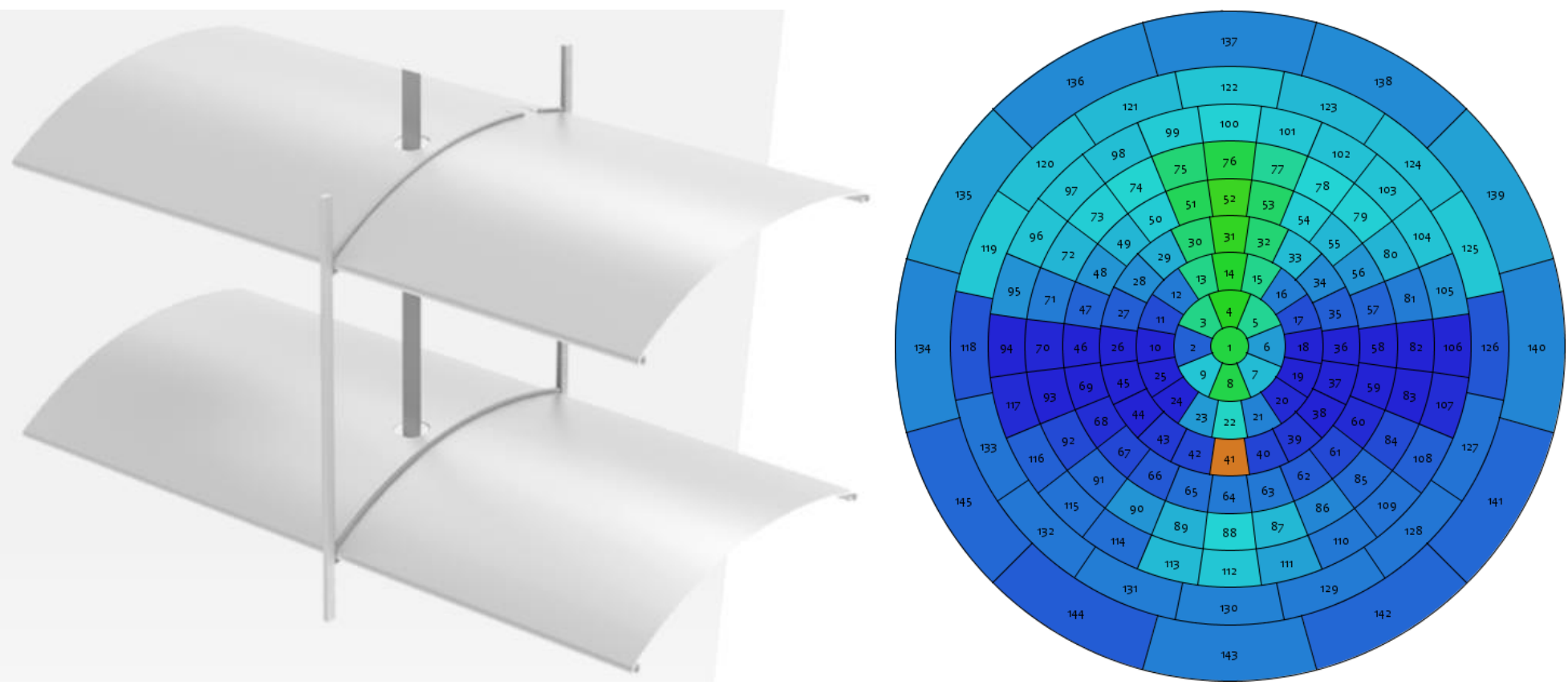

IEA SHC Task 61 / EBC Annex 77: Integrated Solutions for Daylighting and Electric Lighting 


\section{Solar Heating and Cooling Technology Collaboration Programme (IEA SHC)}

The Solar Heating and Cooling Technology Collaboration Programme was founded in 1977 as one of the first multilateral technology initiatives ("Implementing Agreements") of the International Energy Agency.

Our mission is "Through multi-disciplinary international collaborative research and knowledge exchange, as well as market and policy recommendations, the IEA SHC will work to increase the deployment rate of solar heating and cooling systems by breaking down the technical and nontechnical barriers."

IEA SHC members carry out cooperative research, development, demonstrations, and exchanges of information through Tasks (projects) on solar heating and cooling components and systems and their application to advance the deployment and research and development activities in the field of solar heating and cooling.

Our focus areas, with the associated Tasks in parenthesis, include:

- Solar Space Heating and Water Heating (Tasks 14, 19, 26, 44, 54)

- Solar Cooling (Tasks 25, 38, 48, 53, 65)

- Solar Heat for Industrial and Agricultural Processes (Tasks 29, 33, 49, 62, 64)

- Solar District Heating (Tasks 7, 45, 55)

- Solar Buildings/Architecture/Urban Planning (Tasks 8, 11, 12, 13, 20, 22, 23, 28, 37, 40, 41, $47,51,52,56,59,63,66)$

- Solar Thermal \& PV (Tasks 16, 35, 60)

- Daylighting/Lighting (Tasks 21, 31, 50, 61)

- Materials/Components for Solar Heating and Cooling (Tasks 2, 3, 6, 10, 18, 27, 39)

- Standards, Certification, and Test Methods (Tasks 14, 24, 34, 43, 57)

- Resource Assessment (Tasks 1, 4, 5, 9, 17, 36, 46)

- Storage of Solar Heat (Tasks 7, 32, 42, 58, 67)

In addition to our Task work, other activities of the IEA SHC include our:

$>$ SHC Solar Academy

$>$ Solar Heat Worldwide, annual statistics report

$>\mathrm{SHC}$ International Conference

\section{Our members}

Australia

Austria

Belgium

Canada

CCREEE

China

Denmark

EACREEE

ECREEE

European Commission

European Copper Institute
France
Germany
International Solar Energy Society
Italy
Netherlands
Norway
Portugal
RCREEE
SACREEE

SICREEE

Slovakia

South Africa

Spain

Sweden

Switzerland

Turkey

United Kingdom

For more information on the IEA SHC work, including many free publications, please visit www.iea-shc.org. 


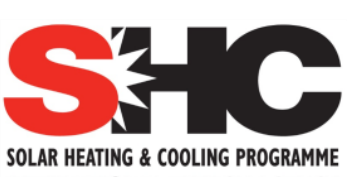

INTERNATIONAL ENERGY AGENCY

\section{Analysis and evaluation of BSDF characterization of daylighting systems}

Editor: David Geisler-Moroder

Authors: Peter Apian-Bennewitz, Jan de Boer, Bruno Bueno, Bertrand Deroisy, Yuan Fang, David Geisler-Moroder, Lars O. Grobe, Jacob C. Jonsson, Eleanor S. Lee, Zhen Tian, Taoning Wang, Gregory J. Ward, Helen Rose Wilson, Yujie Wu

Date: October 2021

T61.C.2.2 - A Technical Report of Subtask C

DOI: 10.18777/ieashc-task61-2021-0012

The contents of this report do not necessarily reflect the viewpoints or policies of the International Energy Agency (IEA) or its member countries, the IEA Solar Heating and Cooling Technology Collaboration Programme (SHC TCP) members or the participating researchers. 
AUTHORS (in alphabetical order)

Peter APIAN-BENNEWITZ

pab advanced technologies Ltd

Gilgenmatten 44

79114 Freiburg

Germany

apian@pab.eu

\section{Jan DE BOER}

Fraunhofer-Institut für Bauphysik IBP

Nobelstraße 12

70569 Stuttgart

Germany

jan.deboer@ibp.fraunhofer.de

\section{Bruno BUENO}

Fraunhofer Institute for Solar Energy Systems

Heidenhofstr. 2,

79110 Freiburg i. Br.

Germany

bruno.bueno@ise.fraunhofer.de

\section{Bertrand DEROISY}

Belgian Building Research Institute

Avenue P. Holoffe 21

1342 Limelette

Belgium

bertrand.deroisy@bbri.be

\section{Yuan FANG}

Fraunhofer-Institut für Bauphysik IBP

Nobelstraße 12

70569 Stuttgart

Germany

yuan.fang@ibp.fraunhofer.de

David GEISLER-MORODER

Bartenbach $\mathrm{GmbH}$

Rinner Strasse 14

6071 Aldrans

Austria

david.geisler-moroder@bartenbach.com

Lars Oliver GROBE

Lucerne University of Applied Sciences and Arts

Technikumstrasse 21

6048 Horw

Switzerland

larsoliver.grobe@hslu.ch
Jacob C. JONSSON

Lawrence Berkeley National Laboratory

1 Cyclotron Road

Berkeley, California 94720

United States of America

jcjonsson@lbl.gov

Eleanor S. LEE

Lawrence Berkeley National Laboratory

1 Cyclotron Road

Berkeley, California 94720

United States of America

eslee@lbl.gov

\section{Zhen TIAN}

School of Architecture

Hunan University

Changsha, Hunan 410082

P.R.China

zhentian@hnu.edu.cn

\section{Taoning WANG}

Lawrence Berkeley National Laboratory

1 Cyclotron Road

Berkeley, California 94720

United States of America

taoningwang@lbl.gov

\section{Greg WARD}

Anyhere Software

950 Creston Rd.

Berkeley, California 94708

United States of America

gregoryiward@gmail.com

\section{Helen Rose WILSON}

Fraunhofer Institute for Solar Energy Systems

Heidenhofstr. 2,

79110 Freiburg i. Br.

Germany

helen.rose.wilson@ise.fraunhofer.de

\section{Yujie WU}

EPFL - LESO-PB

Station 18

$\mathrm{CH}-1015$ Lausanne

Switzerland

yujie.wu@epfl.ch 


\section{KEYWORDS}

Bidirectional scattering distribution function (BSDF), daylighting system characterization, complex fenestration systems, round robin test, daylight performance metrics, evaluation and comparison.

\section{ACKNOWLEDGEMENTS}

The authors thank their respective funding agencies for supporting their work:

- BBRI received funding from Vlaio (Flanders) through the project VIS IV Intelligente Lichtregelsystemen (H8C.2017.0932).

- The work at Bartenbach $\mathrm{GmbH}$ was supported by the Federal Ministry for Climate Action, Environment, Energy, Mobility, Innovation and Technology (BMK) through the IEA Research Cooperation program managed by the Austrian Research Promotion Agency FFG (project 864136).

- The work at EPFL is financially supported by the Swiss Innovation Agency Innosuisse (CTI.2014.0119) and is part of the Swiss Competence Center for Energy Research (SCCER FEEB\&D).

- Fraunhofer IBP received funding by the German Federal Ministry of Economic Affairs and Energy (BMWI) through the project "EnOB: MEET Lichtplanung - Methoden zur effektiven Erschließung von Energieeinsparpotentialen in der Kunst- und Tageslichtplanungspraxis von Gebäuden" managed by the Project Management Jülich PTJ.

- The work of Fraunhofer ISE was supported by a Fraunhofer ICON Grant.

- Research at Hunan University was supported by the National Natural Science Foundation of China (Grant Number 51978429) and the Hunan Province Innovation Development Program (2020TP1009 and 2020RC4045).

- The work at the Lawrence Berkeley National Laboratory and Anyhere Software was supported by the Assistant Secretary for Energy Efficiency and Renewable Energy, Building Technologies Office of the U.S. Department of Energy under Contract No. DE-AC02-05CH11231.

- Research at Lucerne University of Applied Sciences and Arts was supported by the Swiss Innovation Agency Innosuisse, grant number 1155000149, as part of the Swiss Competence Center for Energy Research SCCER FEEB\&D.

The authors would also like to thank Robert Weitlaner (Robert.Weitlaner@hella.info) from HELLA Sonnen- und Wetterschutztechnik GmbH and Jan Berman (Jan.Berman@mechoshade.com) from Mechoshade Systems, Inc. for their in-kind contributions of shade materials for the round robin test. 


\section{PREFACE}

Lighting accounts for approximately $15 \%$ of the global electric energy consumption and $5 \%$ of greenhouse gas emissions. Growing economies, higher user demands for quality lighting and rebound effects as a result of low priced and more versatile electric lighting continuously still lead to an absolute increase of lighting energy consumption. More light is used, often less consciously.

Especially the electric lighting market but as well the façade, daylighting und building automation sectors have seen significant technological developments in the past decade. However, these sectors still act mainly independent of each other, leaving out big potentials lying in a better technology and market integration. This integration is on the one hand beneficial to providing better user-centered lighting of indoor spaces. On the other hand, it can contribute significantly to the reduction of worldwide electricity consumptions and $\mathrm{CO} 2$-emissions, which is in line with several different governmental energy efficiency and sustainability targets.

IEA SHC Task 61 / EBC Annex 77 "Integrated Solutions for Daylighting and Electric Lighting - From component to system efficiency" therefore pursues the goal to support and foster the better integration of electric lighting and daylighting systems including lighting controls with a main focus on the nonresidential sector. This includes the following activities:

- Review relation between user perspective (needs/acceptance) and energy in the emerging age of "smart and connected lighting" for a relevant repertory of buildings.

- Consolidate findings in use cases and "personas" reflecting the behavior of typical users.

- Based on a review of specifications concerning lighting quality, non-visual effects as well as ease of design, installation and use, provision of recommendations for energy regulations and building performance certificates.

- Assess and increase robustness of integrated daylight and electric lighting approaches technically, ecologically and economically.

- Demonstrate and verify or reject concepts in lab studies and real use cases based on performance validation protocols.

- Develop integral photometric, user comfort and energy rating models (spectral, hourly) as prenormative work linked to relevant bodies: CIE, CEN, ISO. Initialize standardization.

- Provide decision and design guidelines incorporating virtual reality sessions. Integrate approaches into widespread lighting design software.

- Combine competencies: Bring companies from electric lighting and façade together in workshops and specific projects. Hereby support allocation of added value of integrated solutions in the market.

To achieve this goal, the work plan of IEA SHC Task 61 / EBC Annex 77 is organized according to the following four main subtasks, which are interconnected by a joint working group:

- Subtask A:

- Subtask B:

- Subtask C:

- Subtask D:

- Joint Working Group:
User perspective and requirements

Integration and optimization of daylight and electric lighting

Design support for practitioners (Tools, Standards, Guidelines)

Lab and field study performance tracking

Evaluation tool \& VR Decision Guide 


\section{EXECUTIVE SUMMARY}

This technical report summarizes the current state of the art in the field of characterization of daylighting and shading systems by bidirectional scattering distribution functions (BSDFs) and documents the results of an inter-laboratory round robin test. It is the result of collaborative work conducted by members of the IEA SHC Task 61 / EBC Annex 77, Subtask C2.

In the first part, various methods for generating BSDF data sets for shading and daylighting systems as applied by different laboratories are documented and compared. Worldwide, several institutes provide the service of measuring and simulating BSDF data for such fenestration systems. However, the labs use various measurement devices as well as different generation workflows with their respective advantages and disadvantages. At the same time, different daylight simulation tools require different BSDF data formats for importing daylighting and shading systems into the software. The aim here is to highlight the pros and cons of the different approaches and to provide an overview of used measurement instruments and simulation software tools.

In the second part, a round robin test performed among the project participants is documented. The objective was to assess the comparability of BSDF data sets generated by various laboratories for the same shading or daylighting system as well as - for practical use in daylighting design even more important - the comparability of daylight performance metric evaluations based on these data. One outdoor venetian blind system and one interior textile roller blind were selected representing widely used shading and glare protection systems. The contributing laboratories generated BSDF data sets for samples of these two systems according to their usual routine. The data sets were analyzed and applied in daylighting simulations to evaluate point-in-time and annual daylight performance metrics. The overall result of the comparison shows that there is good agreement between the BSDF data sets provided by the different laboratories. 


\section{Contents}

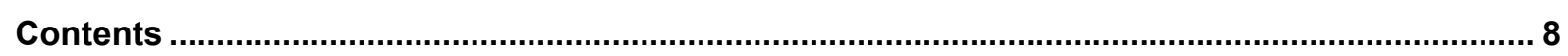

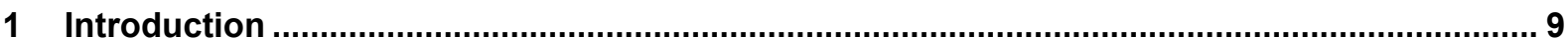

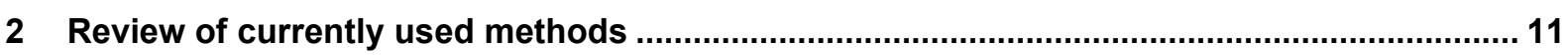

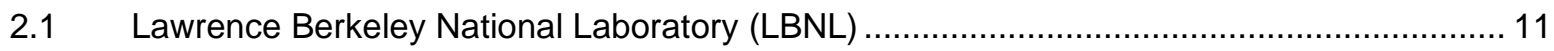

2.2 Lucerne University of Applied Sciences and Arts HSLU ........................................... 14

2.3 Fraunhofer Institute for Solar Energy Systems ISE .................................................. 18

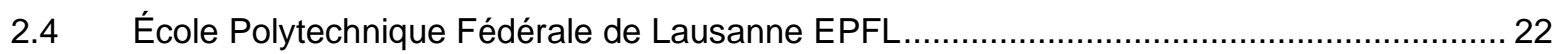

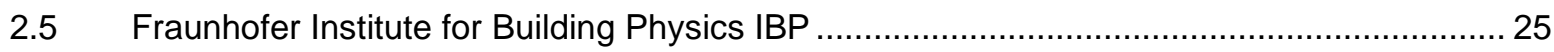

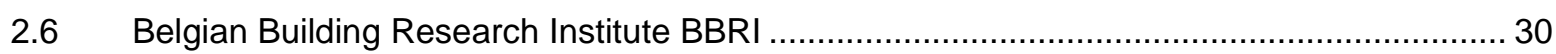

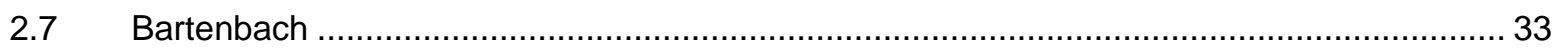

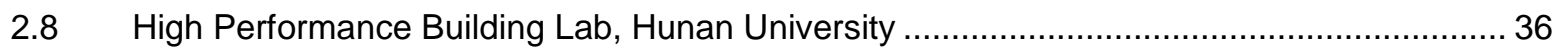

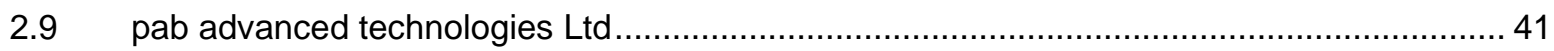

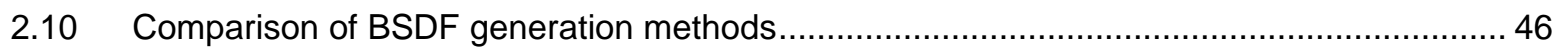

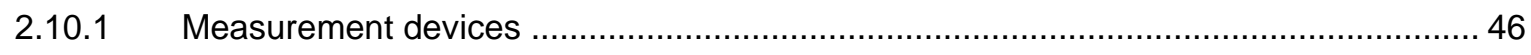

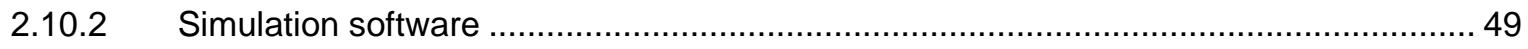

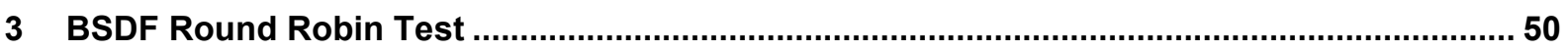

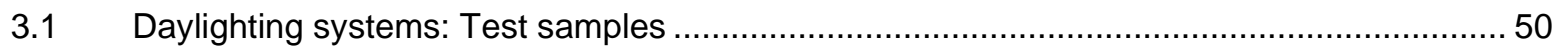

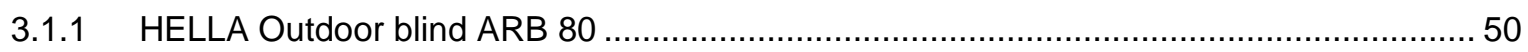

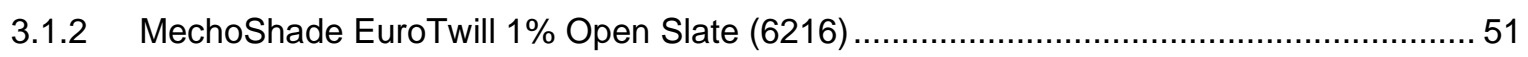

3.2 Contributing laboratories and provided data sets ...................................................... 52

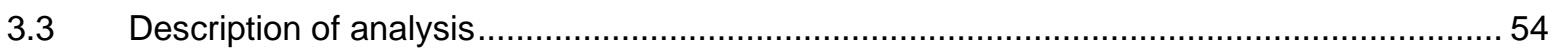

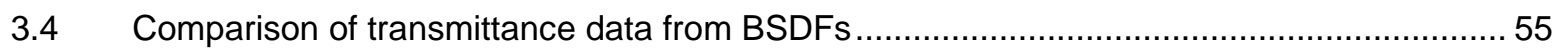

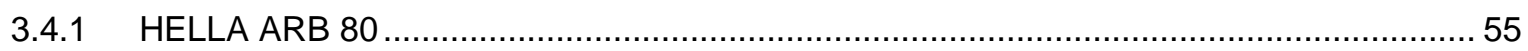

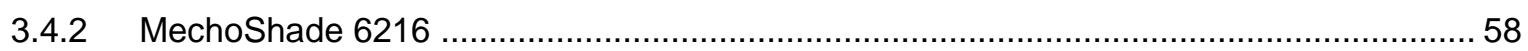

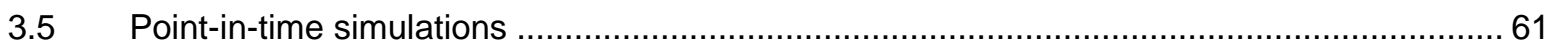

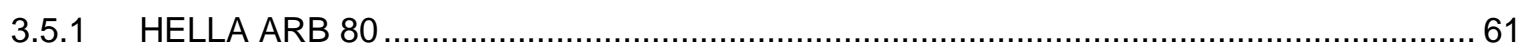

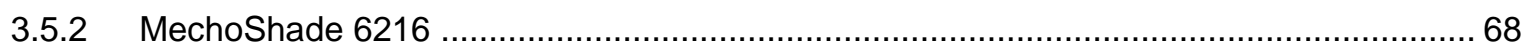

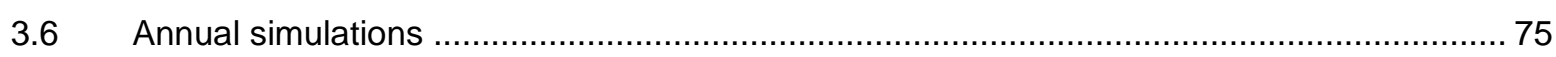

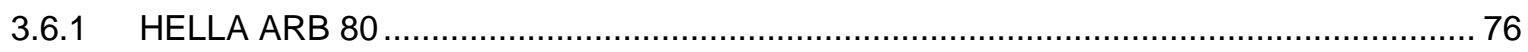

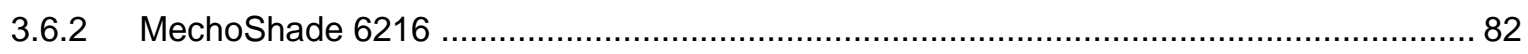

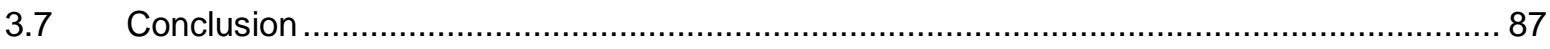




\section{Introduction}

Standardized methods for characterizing angle-dependent, solar-optical properties of transparent glazing for windows are well established, i.e., visible and solar transmittance, absorptance, reflectance, solar heat gain coefficient. Standardized methods do not exist however for "optically complex" or light scattering, shading and daylighting systems, which in turn makes objective evaluation of energy performance, daylighting, comfort, and other building performance qualities almost impossible. Simplified methods for characterizing complex fenestration systems (CFS) have been developed based on normal/normal, normal/hemispherical, and diffuse/hemispherical transmittance measurements. These methods have found their way into European standards ${ }^{1}$, but are of limited use and can contribute to significant error in evaluations of certain aspects of building performance.

In 1994, Klems ${ }^{2}$ proposed that the solar heat gain coefficient (SHGC) of optically complex fenestration systems be calculated based on a $145 \times 145$ discretization of incident and exiting hemispheres. This hemispherical basis subdivision scheme, which yields approximately equal irradiances for each patch at constant radiance, is used in simulation tools such as WINDOW7 ${ }^{3}$ or RADIANCE ${ }^{4}$ to describe a system's bidirectional scattering distribution function (BSDF, i.e., the angle-dependent, solar-optical properties of the system).
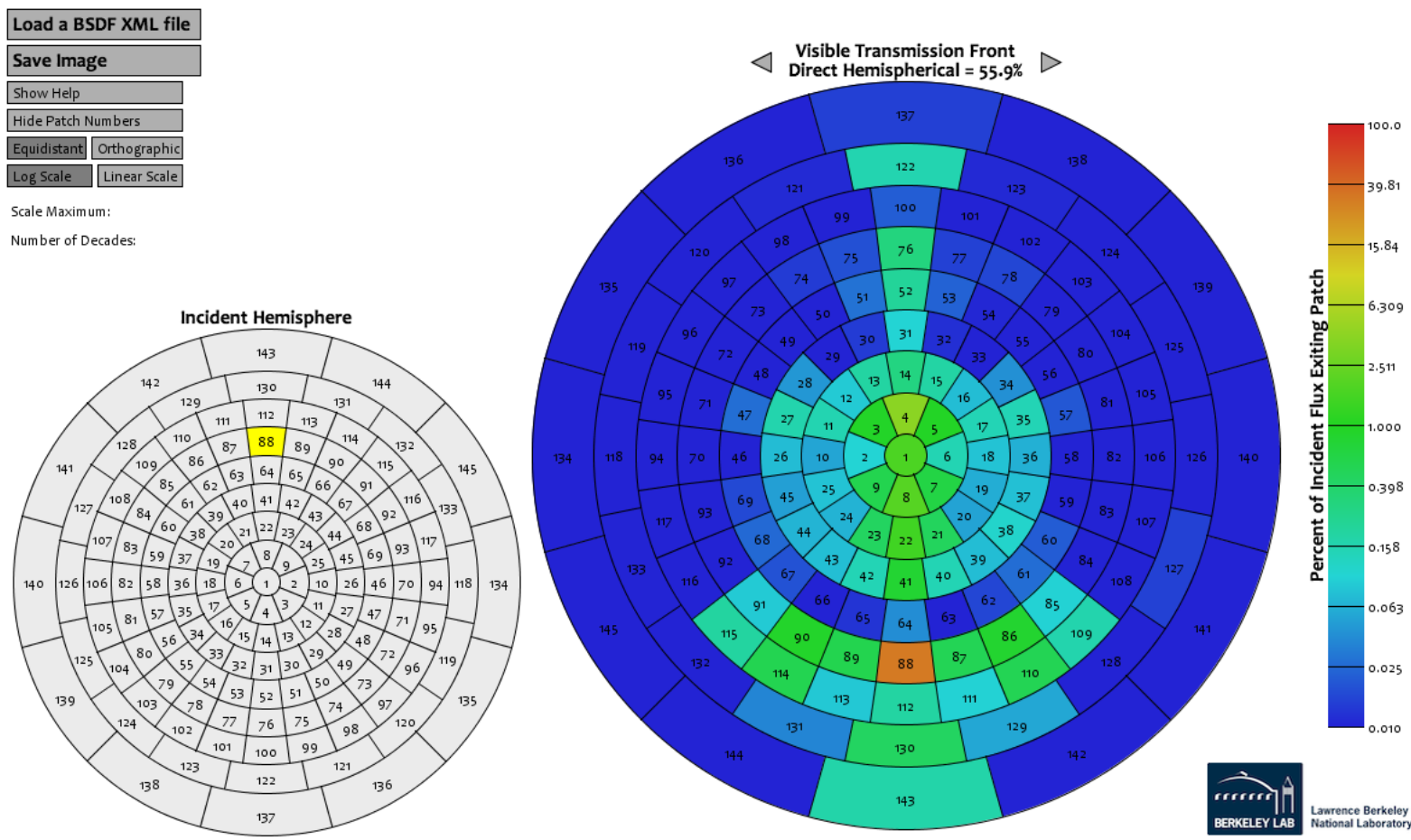

Figure 1: Klems' discretization of incident and exiting hemispheres into 145x145 patches (Screenshot of LBNL's BSDF Viewer).

\footnotetext{
1 DIN EN 14501:2021-09, "Blinds and shutters - Thermal and visual comfort - Performance characteristics and classification", 2021.

2 J.H. Klems, "A new method for predicting the solar heat gain of complex fenestration systems", ASHRAE Transactions, $100(1), 1994$.

3 LBNL, WINDOW7, online: https://windows.Ibl.gov/software/window

${ }^{4} \mathrm{G}$. Ward, R. Shakespeare, "Rendering with Radiance", Morgan Kaufmann Publishers, 1998.
} 
Using these BSDF, it is possible to efficiently integrate shading and daylighting systems in building energy simulations of thermal performance (i.e., solar heat gains through a window with a shade attachment) even without detailed information of the system's geometric or material properties. However, the Klems' subdivision of the hemisphere where every patch corresponds to an average solid angle of $0.043 \mathrm{sr}$ (i.e., a cone with a $2 \times 6.7^{\circ}$ apex angle) must be handled with care when used to compute other performance parameters that require accurate spatial distribution of illuminance or irradiance in the indoor space (such as annual sunlight exposure (ASE), discomfort glare, thermal comfort).

In 2000, the IEA SHC Task 21 "Daylight in Buildings: Design Tools and Performance Analysis" proposed a different hemispherical basis for BSDF characterization of shading and daylighting materials that better met the requirements for daylight performance analysis ${ }^{5}$. For incident directions, Tregenza's sky hemispherical subdivision was used ${ }^{6}$. In contrast to Klems' basis, the 145 directions are distributed to cover approximately equal solid angles. However, as for the Klems basis, they only coarsely discretize the hemisphere of incident directions. For the exiting hemisphere, a non-uniform grid was proposed to minimize the data file size, where areas of steep gradients (e.g., BSDF exiting angles where specular transmission occurs) were required to be measured with a resolution of at least $1^{\circ}$ (apex angle). However, no further details or practical advice on how to handle the resulting, unstructured data were given.

With forward specular and scattering systems, these coarse representations may lead to significant errors. To overcome this, a variable resolution BSDF approach was introduced by Ward et al..$^{7}$ that enables high accuracy via fine angular resolution for steep gradients of the BSDF while providing a coarse structure in areas with flat gradients. Lee et al. ${ }^{8}$ showed that with this so-called "tensor-tree" approach, simulations of complex fenestration systems can be realized at high accuracy. Detailed information about BSDF basics, nomenclature, and resolution schemes are given in the IEA SHC Task 61 's white paper on BSDF generation procedures for daylighting systems ${ }^{9}$.

\footnotetext{
${ }^{5}$ IEA SHC Task 21, "Daylight in Buildings, A Source Book on Daylighting Systems and Components", Lawrence Berkeley National Laboratory, 2000.

${ }^{6} \mathrm{CIE}$, "Guide to Recommended Practice of Daylight Measurement". CIE Publication 108, Vienna, 1994

7 G. Ward, M. Kurt, N. Bonneel, "Reducing Anisotropic BSDF Measurement to Common Practice," Workshop on Material Appearance Modeling, 2014

${ }^{8}$ E.S. Lee, D. Geisler-Moroder, G. Ward, "Modeling the direct sun component in buildings using matrix algebraic approaches: Methods and validation", Solar Energy 160 (2018): 380-395.

${ }_{9}$ D. Geisler-Moroder, E.S. Lee, G. Ward, B. Bueno, L.O. Grobe, T. Wang, B. Deroisy, H.R. Wilson. BSDF generation procedures for daylighting systems. White paper. T61.C.2.1 - A Technical Report of Subtask C, IEA SHC Task 61 / EBC Annex 77. https://task61.iea-shc.org/publications. 2021.
} 


\section{Review of currently used methods}

Several laboratories provide the service of generating BSDF data for shading and daylighting systems. However, the institutes use various measurement devices as well as different BSDF generation workflows with their respective pros and cons. At the same time, different daylight simulation tools require different BSDF data formats for importing daylighting systems into the software.

In this section, these various methodologies for generating BSDF data for shading and daylighting systems are documented by the different institutions and compared. The aim is to highlight the pros and cons of the different approaches and to provide support with an assessment of which approach is suitable for the characterization of shading and daylighting systems. Note that many of the institutions use different methods to generate BSDFs for the same system, depending on analysis objective.

\subsection{Lawrence Berkeley National Laboratory (LBNL)}

by Eleanor S. Lee, Taoning Wang, C. Jacob Jonsson, Lawrence Berkeley National Laboratory, California, USA; Gregory J. Ward, Anyhere Software

The Lawrence Berkeley National Laboratory (LBNL) generates BSDF data using a combination of measured data, empirically-derived models, and/or ray-tracing, depending on the type of system being characterized. As of November 2018, all BSDF data that are publicly available to date through the software tool WINDOW version 7 and LBNL's complex glazing database (CGDB) have been generated for an intended resolution of $145 \times 145$ defined by the Klems basis ${ }^{10}$ (referred to in this section as "lowresolution" BSDF data). Separately, research activities have been underway to define and validate methods for generating BSDF data with a much higher angular resolution (up to $0.5^{\circ}$ apex angle). These activities will continue in collaboration with this IEA Task.

\section{Procedure for generating low-resolution BSDF data for thin fabric materials and systems made of thin fabric}

Standard procedures for generating low-resolution BSDF data are described in documents developed in collaboration with industry and the Attachments Energy Rating Council11,12,13. The AERC 1.1 standard is intended for fabric materials (i.e., used in woven shades, cellular shades, drapes, and roller shades) and other materials with similar forward scattering properties, such as polymer films, and is summarized as follows:

- Measurements of diffuse and direct-hemispherical, front and back transmittance and reflectance are made at normal incidence using a Perkin-Elmer Lambda 950 spectrometer equipped with a $150 \mathrm{~mm}$ integrating sphere.

- Sample inhomogeneity is assessed using multiple samples in a manufacturing run as described in AERC 1.1. Rotational symmetry of the sample is assessed through visual inspection. A representative sample is then selected for measurement.

- Empirical models (derived from detailed measured datasets of fabrics ${ }^{14}$ ) are used to extrapolate data measured at normal incidence to oblique, off-angle properties.

- If the fabric design differs from the empirically-derived case, then integrating sphere measurements are made at additional angles of incidence using the spectrophotometer with an angle tube accessory ${ }^{15}$.

\footnotetext{
10 See Section 15.1 of Curcija, C. et al., WINDOW Technical Documentation, Lawrence Berkeley National Laboratory, April 2018, link.

11 Attachments Energy Rating Council, "AERC 1.1, Procedures for Determining the Optical and Thermal Properties of Window Attachment Materials", version 1.3, 2018, Appendix C, link.

12 Curcija, C. et al., WINDOW Technical Documentation, Lawrence Berkeley National Laboratory, April 2018, link.

13 AERC WINDOW/THERM Simulation Manual, December 13, 2017, p.45, link.

${ }_{14}$ N. Kotey, J. L. Wright, and M. Collins, "Determining off-normal solar optical properties of drapery fabrics," in ASHRAE Transactions, 115, 2009.

15 J. Slack, D. C. Curcija, and J. C. Jonsson, "Angular tubes spectrometer accessory,"

2013. http://windowoptics.lbl.gov/facilities/spectrophotometers, also link
} 
- Detailed goniophotometer measurements are made for anisotropic materials with highly directional scattering patterns but is not considered necessary for most fabrics.

- Data for direct-direct and direct-diffuse transmittance and reflectance are recorded for the front and back surfaces of the fabric over the VIS-NIR range of $300-2500 \mathrm{~nm}$ at $5 \mathrm{~nm}$ increments minimum.

- For shades with geometry, such as cellular shades, the shade layer geometry is drawn in THERM, the shade material data are selected from the "Shade Material Library" in THERM and associated with the shade geometry ${ }^{13}$. The file is exported to the Radiance genBSDF tool to generate layer properties for three wavelength bands. Visible and solar BSDFs are then combined with integrated thermal IR values (TIR and emissivity) into a single XML file ${ }^{16}$.

\section{Procedure for generating high-resolution BSDF data for thin fabric materials}

- As of 2018, there are no such LBNL standards for generating high-resolution BSDF data. LBNL has developed a number of Radiance tools to generate full BSDF data using either 1) material properties with geometry and the genBSDF ray-tracing tool as described above in in Section 2.1. $1^{17}$ and/or, 2) a detailed set of goniophotometer measurements taken for $10-80$ angles of incidence for forward scattering materials such as thin fabrics.

- With the first method, drawings of the fenestration system may not adequately characterize the actual variations in geometry produced by manufacturing. Three-dimensional scans or profilometer measurements of the manufactured component can be conducted to obtain actual geometry. BSDF data can be produced by genBSDF at a user-specified level of resolution then stored in a more compact, tensor tree format.

- For the second method, measurements are taken with a scanning goniophotometer (pab advanced technologies Itd), which automatically rotates the sample in the theta and phi directions relative to the source. A tungsten halogen light source is used to illuminate a sample up to $76 \times 254 \mathrm{~mm}$ at angles of incidence from $-80^{\circ}$ to $80^{\circ}$. Silicon and InGaAs detectors are combined with filters to measure the visible and near infrared wavelength bands. Set up of the optical bench is dependent on the sample size, size and homogeneity of the sample structures, and thickness of the material. The scanning goniometer is placed in a carefully controlled environment to reduce measurement error due to variations in ambient air temperature. The room housing the goniometer is painted matte black.

- For isotropic samples with rotational symmetry about the normal angle of incidence, measurements are taken at $10^{\circ}$ increments of theta. For anisotropic samples, measurements are taken at additional $15^{\circ}$ increments of phi. If the sample exhibits specular transmission or reflection, detailed measurements are made at and around the peak in either a circular or square pattern.

- To generate a full BSDF dataset, the measured data are interpolated using the Radiance pabopto2bsdf tool and reduced to tensor tree data format suitable for simulation using the bsdf2ttree tools. Because the scanning goniophotometer has a limited angular resolution of $1.5^{\circ}$ apex angle (for fabrics with a micro-scale structure), the Radiance peak extraction primitive ("$\left.a B S D F^{\prime}\right)$ is used during the ray tracing calculation to convert view and shadow rays (along the specular direction) from a $1.5^{\circ}$ solid angle to a ray with a $0.5^{\circ}$ solid angle of the sun orb of higher intensity ${ }^{18},{ }^{19}$.

\footnotetext{
16 Page 16-9 of Curcija, C. et al., WINDOW Technical Documentation, Lawrence Berkeley National Laboratory, April 2018, link.

${ }^{17}$ McNeil, A., Jonsson, C.J., Appelfeld, D., Ward, G. and Lee, E.S., 2013. A validation of a ray-tracing tool used to generate bi-directional scattering distribution functions for complex fenestration systems. Solar Energy 98: 404-414. ${ }_{18}$ Ward, G.J., Wang, T., Geisler-Moroder, D., Lee, E.S., Grobe, L.O., Wienold, J., Jonsson, J.C., 2021. Modeling specular transmission of complex fenestration systems with data-driven BSDFs. Building and Environment 196: 107774.

${ }^{19}$ Geisler-Moroder, D., Ward, G.J., Wang, T., Lee, E.S., 2021. Peak extraction in daylight simulations using BSDF data. Proceedings of Building Simulation 2021, International Building Performance Simulation Association, Bruges, September 1-3, 2021.
} 


\section{Discussion: Pros and Cons}

The main benefit of the AERC method is simplicity. From a small set of relatively inexpensive measurements, we are able to derive a suitable BSDF for simulation. However, we are making the assumption that our mathematical model fits different shade materials and weaves, which requires periodic confirmation as new materials are introduced to the market. Further, the exact spatial distribution of the transmitted light may not be sufficiently resolved for critical analysis of glare and similar visual effects.

The genBSDF calculation of scattering properties is the most flexible and permits us to handle any system that can be adequately described as a geometric model. This includes shading and daylighting devices that are envisioned but not yet built, or variants on existing systems. The level of geometric detail is important and can be difficult to determine as mentioned above. Furthermore, the constituent materials are very important, and should themselves be well-characterized or measured by a gonioreflectometer, so costly lab instruments may still be required.

Careful and complete BSDF photometry is the ideal starting point, but existing instruments come with their own inherent limitations. In the case of the pab-opto goniophotometer, it is difficult to measure grazing incident and scattered angles past $80^{\circ}$ from surface normal, so data in these regions must be extrapolated, which is difficult to do reliably. It is also difficult to resolve very small scattering angles without use of a laser source, which would be monochromatic and introduce other limitations. Finally, measurement time is an issue that we overcome somewhat with our advanced interpolation method, but not sufficiently enough to characterize many systems a day, as would be required in a commercial setting.

Ultimately, we need some combination of measurements, geometric models, and mathematical fits to shading and daylighting device classes to drive our calculations, which is why we have adopted this multi-tiered approach. 


\title{
2.2 Lucerne University of Applied Sciences and Arts HSLU
}

\author{
by Lars O. Grobe, HSLU, Switzerland
}

The generation of BSDF data at Lucerne University of Applied Sciences and Arts (HSLU) aims at

- the characterization of fenestration systems and their components to support product development, optimization, and product selection, and

- the modelling of such systems to evaluate their performance in the building context.

While the former objective purely relies on measurements and demands custom procedures depending on the sample and its intended application, the latter implies additional processing and modeling steps such as

- fitting BSDF models to measured BSDF, or the

- compilation of the measured BSDF into data-driven models.

In cases when measuring the fenestration BSDF is not feasible, e.g., if a large number of geometric variants shall be tested, the system model is computationally generated from a geometric description of the fenestration system, combined with data-driven or fitted models of its components' reflection and transmission properties.

A publicly accessible library of simulation models of high directional resolution, in an XML-format compatible to Radiance, and variants employing the Klems angular basis is continuously enhanced ${ }^{20}$.

\section{Characterization of fenestration systems and system components}

HSLU focuses on the characterization of fenestration featuring irregular light scattering properties employing a scanning gonio-photometer ${ }^{21}$ (PG2, pab advanced technologies), operated in configurations that are adapted to the sample properties and the required information to be obtained. This focus implies that no fixed standard protocol is enforced in the measurements, and that currently no integrating transmission and reflection measurements are performed on a regular basis.

Two alternative light sources are operated. A halogen lamp is employed for measurements in the visible light spectrum. Near-infrared radiation is excluded by a hot-mirror to limit the illuminating spectrum to $\lambda$ $\approx 350 \mathrm{~nm}$ to $750 \mathrm{~nm}$, resulting in a correlated color temperature $(C C T)$ of $\approx 2700 \mathrm{~K}$. For solar measurements, a Xenon arc-lamp is employed. Its emission spectrum corresponds to a correlated color temperature $(\mathrm{CCT})$ of $\approx 6000 \mathrm{~K}$ and covers wavelengths in the near infrared spectrum. Employing stacks of step filters, the emitted spectrum can be resolved to bands of $50 \mathrm{~nm}$ width.

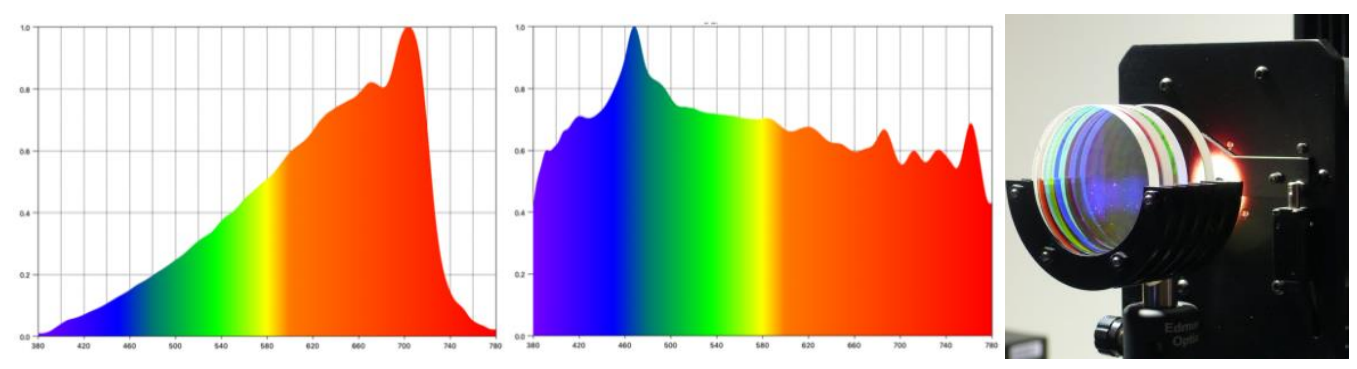

Figure 2: Spectra of halogen (left) and Xenon lamp (center), stack of filters for spectrally resolved measurements (right).

Both sources are installed on an open optical bench, with a translatable focus lens. Translation of the lens determines the apparent size of the source - relating to the beam diameter in the detector plane and the sampling aperture - the beam diameter on the sample - and thereby affects the directional resolution and the coverage of the measurement. For large periodical structures with dimensions up to $15 \mathrm{~mm}$, a slightly diverging beam is configured illuminating a spot of diameter $\approx 70 \mathrm{~mm}$ on the sample, to ensure coverage of at least 4 periods. Uniform, small-structured samples are measured with focus on the detector for maximum directional resolution. For very small samples, or when only a particular region

\footnotetext{
20 https://blog.hslu.ch/solarcontrol

${ }^{21}$ Apian-Bennewitz, P. New scanning gonio-photometer for extended BRTF measurements. In: Proceedings SPIE. 7792 Reflection, Scattering, and Diffraction from Surfaces II. 2010.
} 
on the sample is to be included in the measurement, the beam is focused on the sample to avoid exceeding its dimensions. For small samples, the range of incident directions is limited further since the effective sampling aperture increases with the angle between light source and sample's surface normal $\theta_{i}$. Addressing this limitation with other light sources is currently investigated.

A4-sized samples are preferred. These fit into an automated sample-mount and -changer, allowing to switch between two samples, setting of both incident and outgoing directions $\left(\theta_{i}, \phi_{i}\right)$ and $\left(\theta_{s}, \phi_{s}\right)$, and periodic characterization of the unobstructed illuminating beam. This sample size also allows to cover a wide range of incident and outgoing directions $\left(\theta_{i}\right.$ up to $\left.82.5^{\circ}\right)$. Besides that, the gonio-photometer supports sample sizes ranging from $20 \mathrm{~mm} \times 20 \mathrm{~mm}$ to $1000 \mathrm{~mm} \times 900 \mathrm{~mm}$. This allows for the characterization of small-scale prototypes and local properties on inhomogeneous samples, as well as measurements on samples that cannot be cut to arbitrary sizes or require averaging over an enhanced, representative area. An experimental sample mount was developed for the measurement of retroreflection ${ }^{22}$.
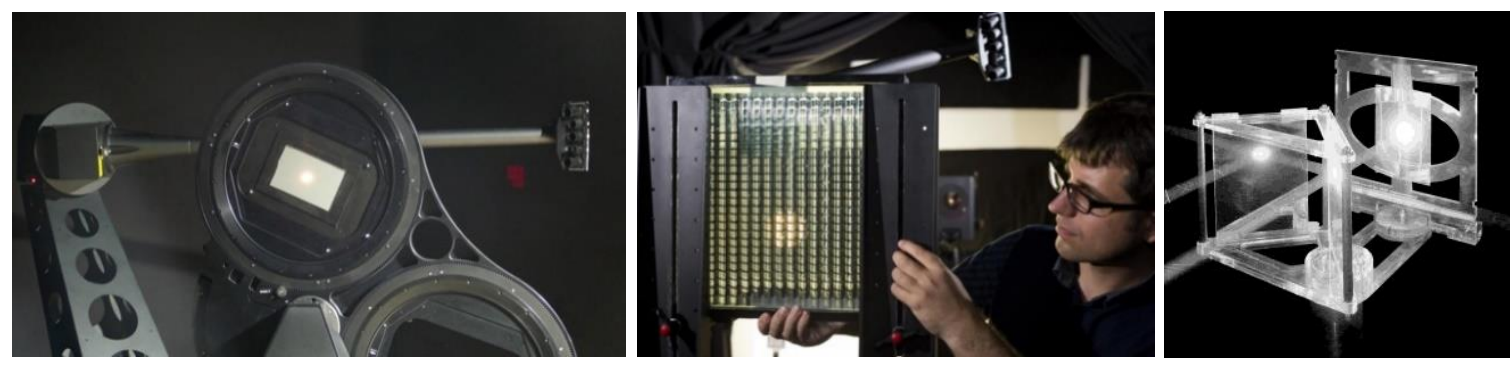

Figure 3: Automated sample-changer (left), mount for large samples up to $1000 \mathrm{~mm} \times 900 \mathrm{~mm}$ (centre) and experimental setup for the characterization of retro-reflective samples (right).

By convention, samples are oriented so that an intended up- or North-direction corresponds to $\phi_{1}=0^{\circ}$, and the normal of the intended outer surface corresponds to $\left(\theta_{i}, \phi_{i}\right)=\left(0^{\circ}, 0^{\circ}\right)$. This convention is based on the assumption that, for most fenestration, a measurement with $\theta_{i}=0^{\circ}$ to $90^{\circ}$ corresponding to the outside could be considered the default minimum requirement. Please note that this convention differs from the frequently used sample orientation employed with LBL Window.

Three detectors aimed at the sample are mounted on a robotic arm. A Silicon cell provides high sensitivity in the visible light range and up to $\approx 1000 \mathrm{~nm}$. A second, identical cell is equipped with a transmission filter to replicate the response of the human eye in photometric measurements. A third detector is based on an Indium-Germanium-Arsenide photodiode and allows for measurement in the near infrared spectrum up to $\approx 2500 \mathrm{~nm}$. The field of view of the detectors exceeds the size of the sample, so that the effective sampling aperture is determined solely by the illuminator. Continuous, mechanical movement of the arm varies the direction of scattered light that is recorded synchronously with the exact position of the detectors.

The measurements are performed as a sequence of scans (measurement sessions), which are programmed through the control software pgc. At the beginning and at the end of each session, the beam is characterized to document any changes in the illuminator's configuration. The measurement resolution is asymmetric for incident and outgoing directions ${ }^{23}$, assuming gradual changes in the outgoing distributions for adjacent incident directions. Incident directions are chosen, according to the sample, either according to specified requirements, or as subsets of the Klems angular basis. For simple, isotropic samples, only $\theta_{\mathrm{i}}$ may be varied with constant incident in-plane angle $\phi$. Most regular structures require the variation of $\phi_{l}$ - depending on symmetries of the sample geometry - from $0^{\circ}$ to $90^{\circ}$ or from $0^{\circ}$ to $180^{\circ}$. For each incident direction, outgoing directions are scanned as a combination of detector paths. In most cases, a spherical path is combined with high-resolution peak scans. If the peak is wide, or the density of the spherical scan is high enough, the location of peaks is automatically found.

22 Grobe LO. Characterization and data-driven modeling of a retro-reflective coating in Radiance. Energy and Buildings 2018 (162):121-133.

23 Apian-Bennewitz P, Scanning gonio-photometers for asymmetric acquisition of fine-structured BSDF. In Eurographics 2014 Workshop on Material Appearance Modeling: Issues and Acquisition, Lyon, France, 2014. 
For samples exhibiting a linear deflection, such as prisms or louvers, an "in-band" scan allows customized high-resolution scanning in and near the scatter plane.

The measured distribution data is stored in a relational database. The data-processing software mountain is used to interpolate, export and to visualize the BSDF for any chosen incident direction, allowing to test for plausibility and indicators of potential errors. Delaunay triangulation is applied to interpolate between the scattered data-points. For each measured distribution, two surface plots are typically generated by mapping the transmission and reflection hemispheres to a polar coordinate system. In the case of samples exhibiting high regular (non-scattered) transmission or mirror-like, specular reflection, a logarithmic scale is applied. To avoid the exaggeration of noise at directions close to grazing (due to the cosine-term in the formulation of the BSDF), the Differential Scattering Function $\mathrm{DSF}=\mathrm{BSDF} \times \cos \left(\theta_{s}\right)$ is plotted rather than the BSDF, and may be further clipped at e.g., $\theta_{s}=90^{\circ} \pm 5^{\circ}$. To generate PDF-formatted, publication-grade surface plots, a custom tool has been developed. Plots of slices through the distributions, e.g., profiles of the DSF in the scattering plane, are generated by mountain and lend themselves especially to the comparison of measurements. Further post-processing steps for the evaluation of the measured sample may include e.g. the separation of directional regions of interest, the interpolation and re-gridding to obtain regularly-spaced data-points.

For archival as well as further processing, mountain allows to export the distributions for each measured incident direction in a tabular text-format following the recommendations of ASTM E2387-0524. Besides the data-table comprising the coordinates of the data-points the measured BSDF (or DSF), the measurement conditions are documented in detail in a set of header-lines in each file.

\section{Data-driven modeling of fenestration by its BSDF}

To generate models for daylight simulation, a tool-chain distributed with Radiance is employed employing interpolation between incident directions (pabopto2bsdf), discretization of the interpolants to a three- (for isotropic) or four-dimensional tensor (for anisotropic light scattering), and data-reduction of the tensor to build a compact data-driven model of adaptive resolution (bsdf2ttree).

The resolution, and the target for the data-reduction algorithm, are chosen according to the sample properties and the intended application of the model. A resolution of 16384 incident and 16384 outgoing directions is the maximum of what can be processed on commonly available hardware, and adequately represents the resolution of the measurement $\left(<1^{\circ}\right.$ to $\left.4^{\circ}\right)$. If no distinct peaks or other sharp features are present in the measurements, e.g., due to diffusing layers or structures, this resolution can be reduced, drastically decreasing the model-generation times. The efficiency of the data-reduction algorithm heavily depends on the presence of large regions without distinct features in the distributions. Samples with regular scattering properties, comprising e.g., a diffuse background and one reflective, one transmissive peak, can therefore be successfully represented by a model with very high initial resolution and data-reduction by $95 \%$ to $98 \%$ - maintaining the full resolution only in the small regions of the peaks. For irregular scattering with often multiple complex features in the BSDF, the target for the data-reduction has to be decreased as no large, continuous regions of low variance exist in the initial tensor ${ }^{25}$.

For calculations relying on the Klems directional basis, low-resolution variants are generated employing the Radiance tool bsdf2klems. To make sure that no peaks or other constraint features in the highresolution model are missed, a sufficient number of samples have to be drawn for each of the Klems patches. Typically, a value of 256 to 512 samples per patch will reliably sample a high-resolution model.

Solar BSDF models can be approximated by the weighted combination of the measured BSDF in the visible and the near infrared spectra. While the combination of Klems models is a simple element-wise multiplication and addition of vectors, the combination of high-resolution models is non-trivial due to their adaptive resolution. Rather than generating combined BSDF models from tensors, this combination is therefore performed at HSLU by "mixing" the models in the simulation employing the Radiance mixfunc primitive.

\footnotetext{
${ }^{24}$ ASTM E2387-05. Standard Practice for Goniometric Optical Scatter Measurements. 2011.

25 Grobe LO, Wittkopf S, Kazanasmaz ZT. High-resolution data-driven models of Daylight Redirection Components. Journal of Facade Design and Engineering. 2017;5(2):101-113.
} 
Modeling fenestration by the combination of the BSDFs of its layers supports the reuse of data. For BSDF models of fixed directional basis, this is implemented e.g., in LBNL Window. If high-resolution models are to be combined, either elaborate ray-tracing is employed or the application of the matrixcomputations to sampled models of variable resolution ${ }^{26}$.

If the geometry of shading systems is known with certainty, the light scattering properties are computed employing the Radiance tool genBSDF. The computed BSDF only for angles that are problematic in the measurement can optionally be combined with the measured BSDF of the system to build hybrid BSDF models $^{27}$. In general, the computational generation of BSDF is favorable

- for large-scale structures that can be precisely described geometrically,

- if the direct measurement of the fenestration's BSDF appears to be not feasible due to e.g., the size of structures, and

- if the fenestration allows multiple configurations, e.g., tilt angles, that would require many measurements.

Besides Radiance, data-driven models for the optical simulation software LightTools have been generated by interpolation and resampling.

\section{Discussion: Pros and Cons}

Relying on full gonio-photometric characterization is elaborate but is of general applicability and avoids the potential errors introduced by models. Acquisition time and effort appear to be acceptable for the current focus on samples with highly irregular scattering properties, and measurements customized to specific questions e.g., by developers. Comparisons with integrating measurements as well as other gonio-photometric setups have confirmed the accuracy of the method. Due to the high configurability of the setup, the main challenge is the documentation of the measurement conditions, and the rigorous checking of the resulting large data-sets. The latter is supported by the design of the device supporting internal cross-checks.

The current practice to sequentially characterize the BSDF in different wavelength bands is not ideal in terms of measurement times. In principle, several sensors could be read out in parallel while performing one scan path, reducing the acquisition times to just a fraction of today's practice. Similarly, there is a not yet leveraged potential to drastically decrease measurement time by adapting the directional resolution of the measurement to that of the intended application. When the aim is e.g., the generation of models employing the Klems directional base, reducing the resolution of the measurement accordingly would drastically reduce the acquisition times, and increase robustness toward e.g., noise. When exact knowledge of peak-structures is desired, e.g., in the case of mirror-like reflection or scattering due to imperfections in refractive structures, dedicated configuration could locally further increase the resolution. While current practice is to extend the applicability of one default configuration as far as possible, optimizations appear to be necessary and feasible if larger batches of measurements are to be performed.

One important limitation of the gonio-photometer's far-field operation is that the sampling aperture is limited, and that its size correlates with the achievable directional resolution. Extending the setup to near-field measurements would overcome this constraint. It would further open a path toward the characterization of light scattering not only as an average BSDF, but as a spatially varying property.

\footnotetext{
${ }^{26}$ Grobe LO. Computational combination of the optical properties of fenestration layers at high directional resolution. Buildings. 2017;7(1).

${ }^{27}$ Krehel M, Grobe LO, Wittkopf S. A hybrid data-driven BSDF model to predict light transmission through complex fenestration systems including high incident directions. Journal of Facade Design and Engineering. 2017;4(3-4):10.
} 


\title{
2.3 Fraunhofer Institute for Solar Energy Systems ISE
}

\author{
by Bruno Bueno and Helen Rose Wilson, Fraunhofer ISE, Germany
}

The generation of BSDF datasets for fenestration systems at Fraunhofer ISE is based on a combination of experimental and numerical techniques. The following description applies to fenestration systems composed of a glazing unit and a shading device, although different complex fenestration systems can be characterized applying ad-hoc workflows. The described workflow differentiates interior, in-between and exterior shading device. The specific workflow depends on whether the shading device is quasihomogeneous (e.g., light-diffusing polymer films) or heterogeneous (e.g., louvered systems), as explained below.

The experimental equipment for optical measurements at Fraunhofer ISE is part of the Testlab Solar Facades and consists of integrating spheres and a 3D-scanning photogoniometer. The integrating spheres and spectrometers to determine spectrally resolved direct-hemispherical transmittance and near-normal-hemispherical reflectance of fenestration materials and systems are accredited according to DIN EN ISO/IEC 17025:2018.

\section{Integrating spheres}

Integrating spheres are used to determine the transmittance and reflectance of fenestration materials and systems over the solar spectral range. An integrating sphere consists of a hollow spherical cavity with its interior covered with a diffuse white reflective coating and with small apertures for entrance, exit and detector ports. For transmittance measurements, a sample is placed at a sphere aperture between the light source and the integrating sphere. A detector located at one of the ports of the sphere measures the spatially integrated scattered light from the sample due to multiple diffuse reflections from the internal sphere surface.

Integrating spheres of different diameters are used to determine spectral transmittance and reflectance for variable incidence angles.

The direct-hemispherical transmittance and reflectance spectra of light-scattering or light-redirecting samples are measured over the spectral range from app. $350 \mathrm{~nm}$ to app. $1800 \mathrm{~nm}$ with a Tec5 diode array spectrometer, using integrating spheres of diameter $620 \mathrm{~mm}$ which are internally coated with sintered PTFE. Direct-hemispherical transmittance is measured with the light incident at angles of incidence between $0^{\circ}$ and $75^{\circ}$ and the sample mounted at the entrance aperture to the sphere. Directhemispherical reflectance is measured with the light incident at angles of incidence between $8^{\circ}$ and $75^{\circ}$ and the sample mounted in a rotatable sample holder within the sphere (Edwards mount). The back of the sample holder consists of a panel of sintered PTFE, which serves as the reflectance reference for the relative reflectance measurements.

For non-scattering samples and scattering samples where lateral spread of light within the sample itself is negligible, the normal-hemispherical and normal-diffuse transmittance and reflectance spectra are measured using a Perkin-Elmer Lambda-900 spectrometer and a $220 \mathrm{~mm}$ integrating sphere also coated internally with PTFE. The fixed angle of incidence is $0^{\circ}$ for the transmittance measurements and $8^{\circ}$ for the reflectance measurements. Normal-normal spectra are calculated as the difference between the relevant normal-hemispherical and normal-diffuse spectra. For light-scattering samples with multiple internal reflections (e.g., light-scattering glass panes), only the normal-normal spectra are determined using this sphere, as lateral light losses would cause significant errors in normal-hemispherical and normal-diffuse spectra for the given sphere and aperture dimensions.

\section{D-scanning photogoniometer}

A 3D-scanning photogoniometer is used to determine BSDF data of fenestration materials and systems. The setup at Fraunhofer ISE (PG2 ${ }^{28}$, pab Advanced Technologies Ltd) consists of an illumination system and a robotic arm that moves a sensor over the surface of a virtual sphere (radius $=1 \mathrm{~m}$ ) around a sample. As the optical axis of the detector is always oriented along a radius of the virtual sphere described by the detector's path, the detector characteristic remains constant for all emergent angles from the sample. Radiation transmitted or reflected by the sample can be measured at almost all emergent angles. Three different sample holders allow the rotation of differently sized samples of area

28 Apian-Bennewitz P. New scanning gonio-photometer for extended BRTF measurements. In: Proceedings SPIE. 7792 Reflection, Scattering, and Diffraction from Surfaces II. 2010. 
up to $500 \mathrm{~mm} \times 500 \mathrm{~mm}$ around two axes relative to the optical axis of the illumination system; one of them is illustrated in Fig. 5. Using this sample holder, it is possible to completely automate a measurement sequence that includes not only the reference beam and sample measurements, but also dark-signal measurements. The large sample holder, as illustrated in Fig. 3 (center image), does not allow automated rotation of a sample in its own plane, but can accommodate samples of area up to 900 $\mathrm{mm} \times 1000 \mathrm{~mm}$. For any source direction the robotic arm allows high-resolution measurements of scatter directions in the transmission and reflection hemispheres. The scanning photogoniometer is equipped with two light sources: halogen and xenon lamps, and different detectors: a $V(\lambda)$ filtered silicon diode (Visible detector), broadband silicon diode, broadband InGaAs diode (NIR detector), broadband $\mathrm{SiC}$ diode (UV detector), and diode array spectrometers (visible and NIR, applying Si and InGaAs diodes, respectively).

The BSDF for the visible spectral range is measured directly with the halogen lamp as the light source and the $\mathrm{V}(\lambda)$ filtered silicon diode as the detector, as its spectral response closely approximates the relatively narrow $V(\lambda)$ function. The beam of the halogen lamp is focused on the detector, such that the illuminating spot on the sample has a diameter of app. $25 \mathrm{~mm}$ at normal incidence. If this spot is not large enough to adequately sample an inhomogeneous test specimen, measurements are made for different positions of the specimen. The exact method for determining the solar BSDF depends on the spectral information available for the investigated sample but relies basically on making a set of BSDF measurements with a sufficient number of different broadband detectors for the intended application purpose. The xenon lamp is used for illumination in this case.

In general, the detector head is initially scanned over the entire sphere surface around the sample, during which peaks in the intensity distribution are identified. These are then scanned with finer spatial resolution to capture sharp peaks and high-gradient variations in reflected or transmitted light intensity.

The incidence angles are selected to take the symmetry properties of the sample into account and the angular resolution needed for the intended application of the BSDF data. For example, for woven fabrics, with orthogonal warp and weft yarns, at least three different phi_in values $\left(0^{\circ}, 90^{\circ}\right.$ and at least one intermediate angle) would represent a minimal selection of azimuthal angles in order to sample directions with extremal transmittance for a square weave. If the weave is rectangular or the sample has rotational symmetry of order 2 , as is the case for the twill sample investigated in the round robin, equivalent phi_in values from an adjacent quadrant would also be needed. It is then possible to obtain the BSDF over the complete hemisphere of incidence angles by reflection or rotation and interpolation, applying the Radiance tools referenced in Section 2.1. Alternatively, azimuthal angles from all quadrants of the incidence hemisphere may be deliberately chosen to verify the correctness of an assumed sample symmetry or to quantify tolerances. A selection of polar angles (theta_in) in $10^{\circ}$ intervals from $0^{\circ}$ to $80^{\circ}$ has proven to be widely applicable for different sample types. However, a $1^{\circ}$ interval near the geometrically estimated cut-off angle is recommended if the measurements are to be used for cut-off angle determination. Including a theta in value of $8^{\circ}$ is also recommended as a near-normal incidence angle that avoids artefacts that may occur at $0^{\circ}$ in transmission due to multiple reflections between sample and detector or lamp optics, and in reflection due to beam obstruction by the detector head.

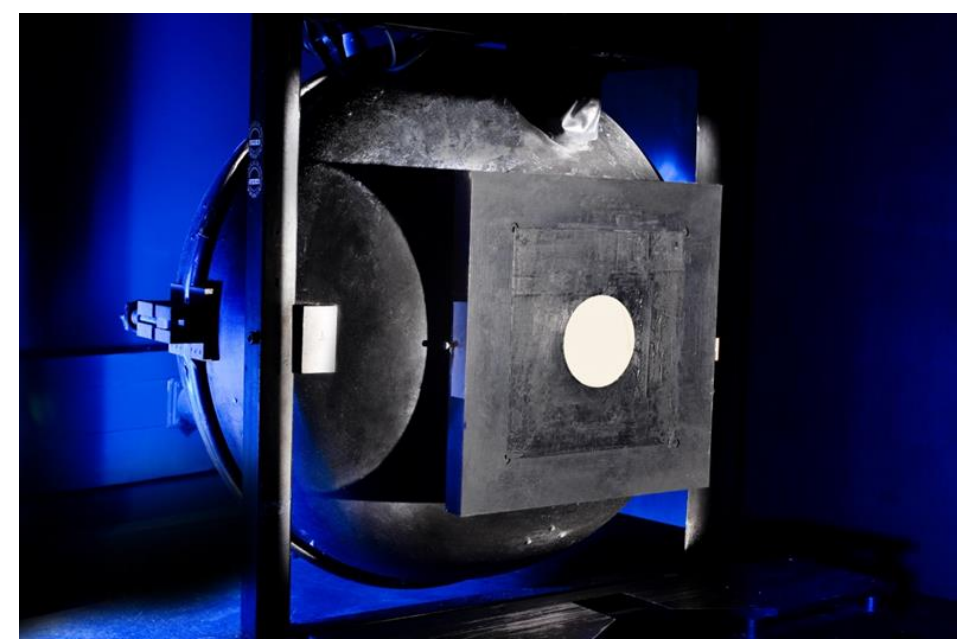

Figure 4: Large integrating sphere $(620 \mathrm{~mm}$ diameter) for angle-dependent direct-hemispherical transmittance measurements of light-scattering samples. The entrance aperture that is visible here has a diameter of $100 \mathrm{~mm}$. Accredited according to DIN EN ISO/IEC 17025 since 2006. C) Fraunhofer ISE 


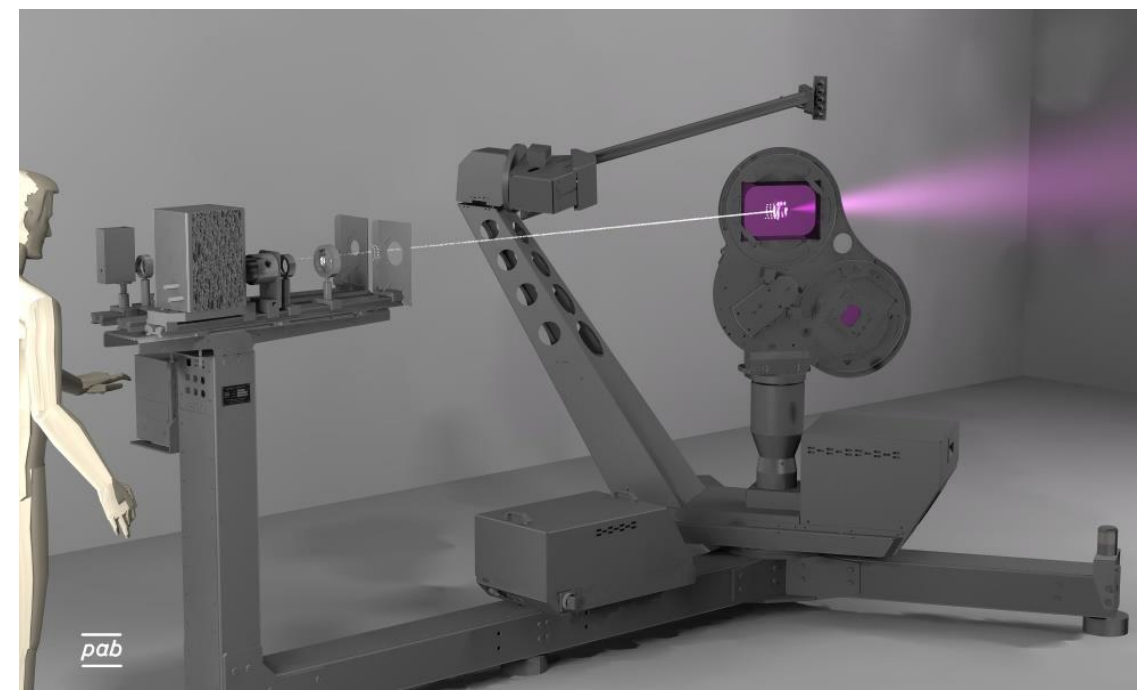

Figure 5: Schematic diagram of the photogoniometer PG2 at Fraunhofer ISE (Freiburg, Germany), showing the light source, the detector head and the phirot2 sample holder for two different samples. ( Peter Apian-Bennewitz, pab advanced technologies Ltd.

\section{Optical characterization of fenestration systems composed of a shading device and a glazing unit}

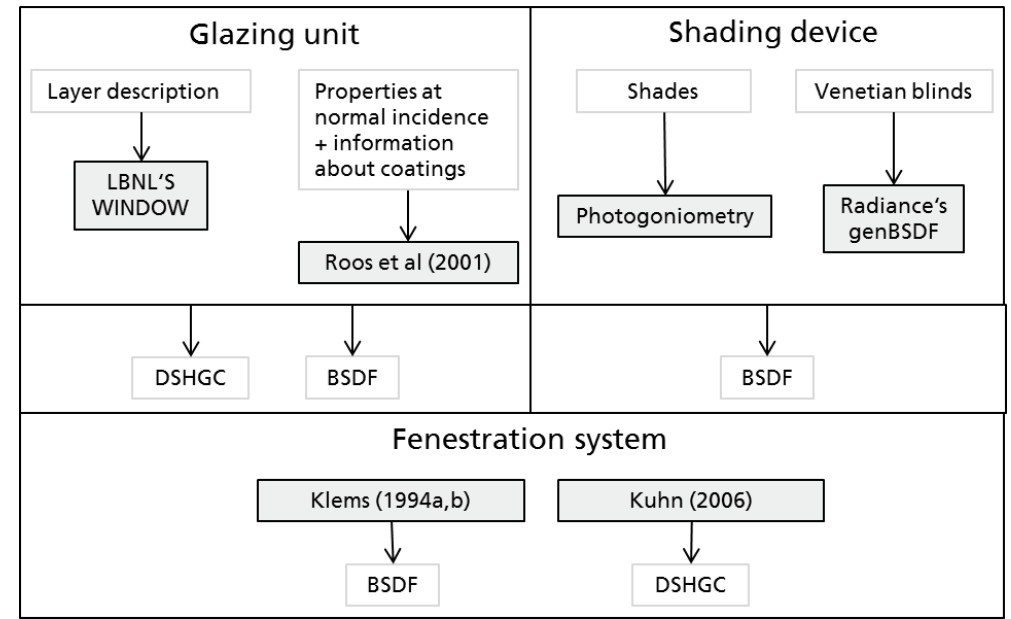

Figure 6: Workflow to obtain BSDF and DSHGC of a fenestration system composed of a glazing unit and a shading device. Source: Bueno et al. ${ }^{29}$.

The Klems method ${ }^{30}$ is applied to calculate the BSDF of the system from the BSDF of the glazing unit and the BSDF of the shading device. The BSDF of the glazing unit (a diagonal matrix) can be generated with the LBNL WINDOW program or calculated from the Roos model ${ }^{31}$ if information about coatings is provided. The BSDF of shading devices can be calculated by the Radiance-based tool genBSDF ${ }^{32}$ if enough information about the optical properties of its components' surfaces is available. Alternatively, a BSDF can be directly measured with a photogoniometer as explained above. These measurements are

${ }^{29}$ B. Bueno, J.M. Cejudo-Lopez, T. E. Kuhn, A general method for the evaluation of the thermal impact of complex fenestration systems in building zones. Energy and Buildings 155 (2017) 43-53.

$30 \mathrm{~J}$. Klems, A new method for predicting the solar heat gain of complex fenestration systems: I. Overview and derivation of the matrix layer calculation, American Society of Heating, Refrigerating and Air- Conditioning Engineers Transactions 100 (1) (1994) 1065-1072.

31 A. Roos, P. Polato, P. A. van Nijnatten, M. G. Hutchins, F. Olive, C. Anderson, Angular-dependent optical properties of low-e and solar control windows: Simulations versus measurements, Solar Energy 69, Supplement 6 (0) $(2001) 15-26$.

32 A. McNeil, C. Jonsson, D. Appelfeld, G. Ward, E. Lee, A validation of a ray-tracing tool used to generate bidirectional scattering distribution functions for complex fenestration systems, Solar Energy 98, Part C (0) (2013) $404-414$ 
mainly limited by the dimensions of the sample, which must be less than $500 \mathrm{~mm} \times 500 \mathrm{~mm}(900 \mathrm{~mm} \times$ $1 \mathrm{~m}$, if automatic rotation in the sample plane is not required). At the same time, the representative period of the macroscopic structure of the sample must be a few times smaller than the illuminated patch, or measurements must be made at different positions on the sample. For this reason, BSDF of slat systems are generally obtained by ray-tracing from the optical properties of the slat surfaces, while the BSDF of e.g., roller blinds can be directly measured with a photogoniometer.

\section{Discussion: Pros and Cons}

The experimental approach at Fraunhofer ISE combines spectral integrating-sphere measurements and spatially resolved broadband BSDF measurements. This allows in-house comparison and validation for spectrally and spatially integrated direct-hemispherical transmittance and reflectance.

The control program of the 3D-scanning photogoniometer allows definition of input parameters over a wide range to adapt the spatial resolution of the output angles according to the desired purpose - from very fine to very coarse. It also allows a large dynamic measurement range for intensity. With the current infrastructure, we can carry out measurement of the central area of large samples (up to $900 \mathrm{~mm} \times 1000$ $\mathrm{mm}$ ). Sequences of input angles can be readily programmed for fully automatic operation after samples have been mounted.

The scan time needed for a single input angle depends on the required resolution of the output angles and the dynamic range of the measured signals. The minimum time for a scan including fine resolution of peaks is about 20 minutes. As the total measurement time depends approximately linearly on the number of input angles, the input angles should be chosen carefully to obtain a BSDF "fit for purpose", taking information on sample symmetry into account. 


\title{
2.4 École Polytechnique Fédérale de Lausanne EPFL
}

\author{
by Yujie Wu, EPFL, Switzerland
}

The generation of BSDF is based on a CCD (charge-coupled device) imaging based Goniophotometer ${ }^{33}$ positioned in a dark room at LESO-PB (Solar Energy and Building Physics Laboratory), EPFL. This device, originally designed to monitor both BTDF and BRDF, has been simplified on its configuration to measure only BTDF for window material and CFS featured with various dimension of sub-structures, in order to maintain its mechanical stability and reliability. The Goniophotometer is equipped with an HMI (Hydrargyrum Medium-arc lodide lamp) light source (Korrigan 1011+, 1.2 kW, 110k Lumens) with color temperature $5600 \mathrm{~K}$, which generates an intense collimated beam on the sample plane through its optical lens groups at $10.3 \mathrm{~m}$ distance. Due to the limited dimension of the Goniophotometer, a CFS sample under test cannot exceed $50 \mathrm{~cm}$ in its diameter to be fixed on the sample disk of the Goniophotometer. The sample disk can rotate both about its center and about the horizontal axis to alter the zenith and azimuth angle of incident light beam relative to the sample plane. An external luxmeter (Pocket-Lux 2B, up to 20,000 lux) is positioned vertically to measure the incident illuminance, which can be converted to projected illuminance incident on the sample plane. Inside the body of the Goniophotometer, a white triangle screen with Lambertian diffusing coating is used to reflect transmitted light in the exiting hemisphere to a calibrated CCD camera, which is optically corrected by filters to measure luminance values of transmitted light based on which BTDF data is generated. For each incident direction, the triangle screen rotates six times about the center axis of sample to cover all the exiting directions of transmitted light.

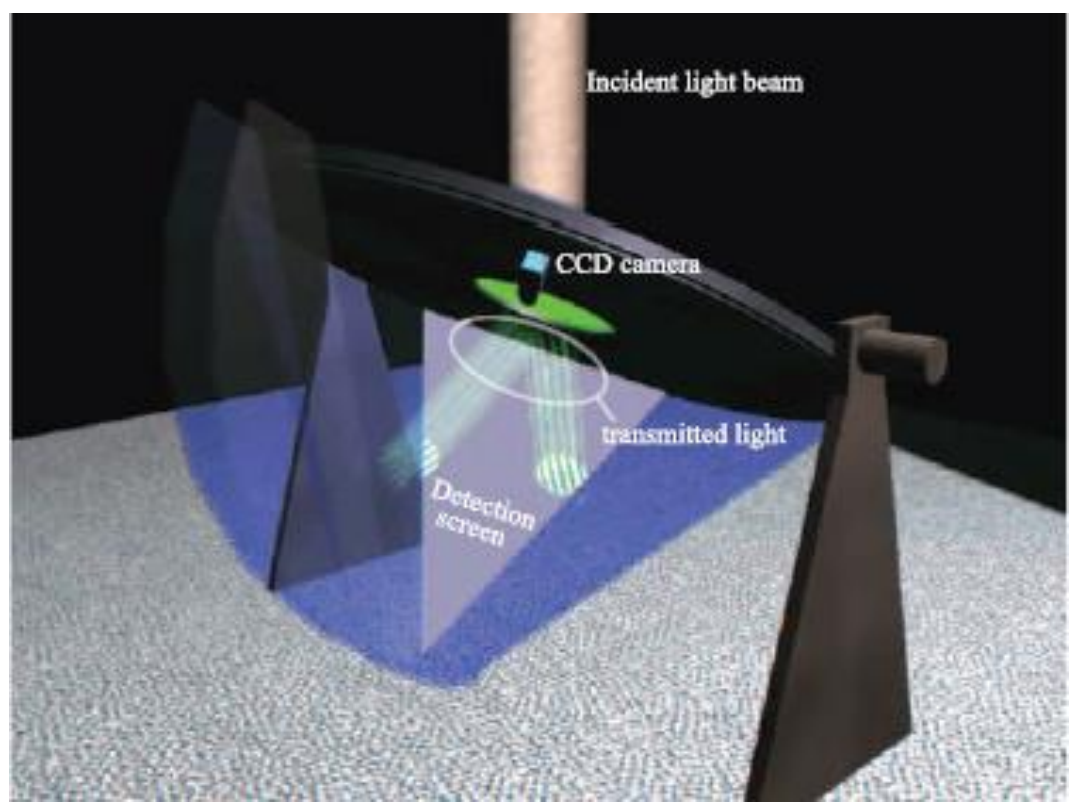

Figure 7: Schematic measurement setup of CCD imaging based Goniophotometer for BTDF monitoring

\footnotetext{
${ }^{33}$ Marilyne Andersen, Christian Roecker, Jean-Louis Scartezzini, Design of a time-efficient video-goniophotometer combining bidirectional functions assessment for transmission and reflection, Solar Energy Materials and Solar Cells, Volume 88, Issue 1, 2005, Pages 97-118.
} 

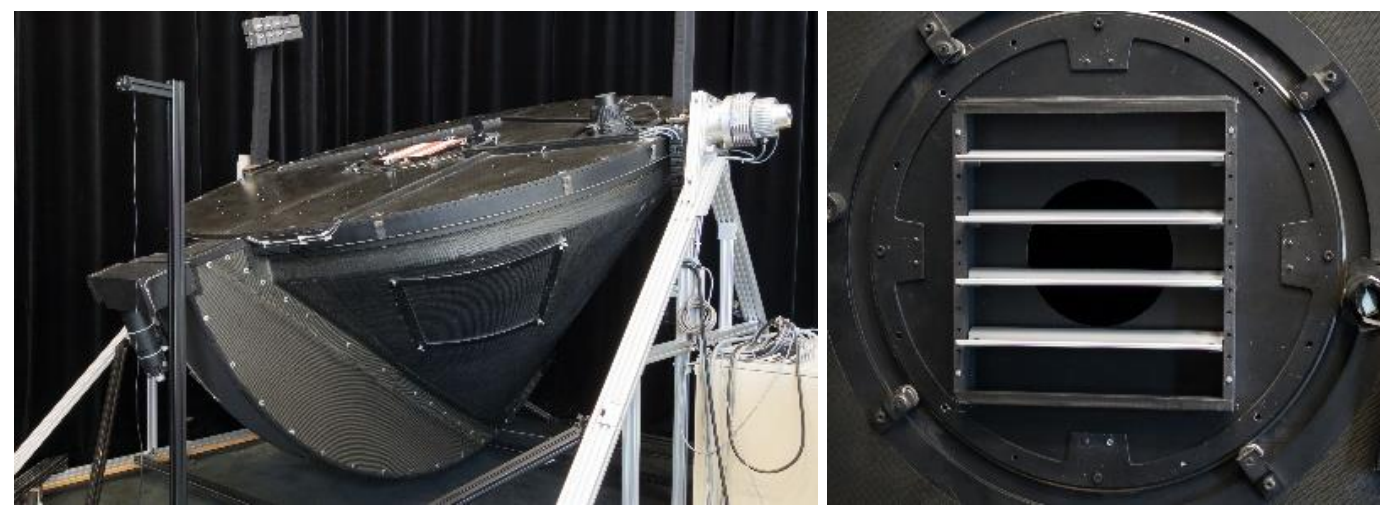

Figure 8: CCD imaging based Goniophotometer for BTDF monitoring

The maximum resolution of the BTDF on the exiting hemisphere that the Goniophotometer can quantify is 1297 directions with $5^{\circ}$ equal steps in zenith and azimuth angle, respectively. Although the number of incident directions can be arbitrary, BTDF measurement commonly employs the Tregenza angular basis with 145 incident directions, which is accomplished automatically by the Goniophotometer via rotation of the sample disk. It takes 5 min to accomplish a measurement from one incident angle. For 145 incident directions, it takes about 12 hours to finish one measurement of BTDF with 145 incident and 1297 exiting directions. If the sample has symmetry about its center or one axis, the time consumption can be reduced by discarding redundant incident directions. The Goniophotometer is equipped with different aperture sizes, from $6 \mathrm{~cm}$ to $15 \mathrm{~cm}$, on the sample disk to fit with CFS with different dimensions in the sub-structure. Although the Goniophotometer is able to monitor BTDF of various CFS, its maximum resolution, especially in the exiting hemisphere, can be limited by the dimension of its sub-structure.

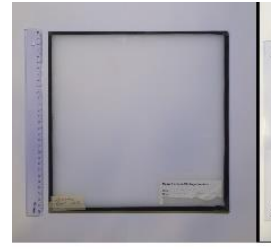

a

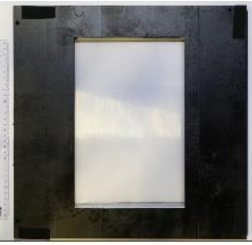

b

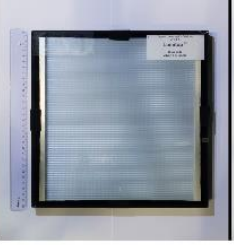

c

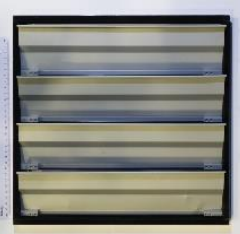

d

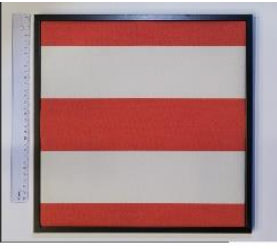

e

Figure 9: CFS samples under measurement a) Opalescent Plexiglas, b) Prismatic panel,

c) Sunlight-redirecting glass, d) Metallic Slats, e) Fabric Blind

The BTDF data generated by the Goniophotometer is stored in the IEA21 format ${ }^{34}$, which is a group of text files storing BTDF data in the exiting hemisphere from each incident direction. The BTDF data can also be transformed into an XML format with defined angular basis to be parsed by the RADIANCE program for lighting simulation. Moreover, the collected BTDF data can be visualized in hemispherical projections from an incident direction, where brightness of shades denotes the magnitude of BTDF data in the exiting hemisphere, or visualized in photometric solids, which clearly illustrates the directional distribution of the BTDF data, as illustrated in the following figures.

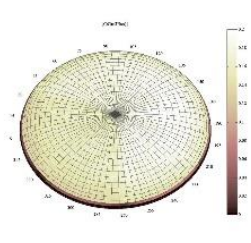

a

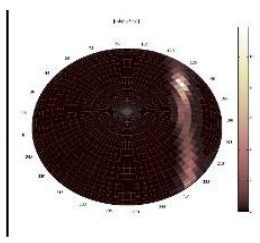

b

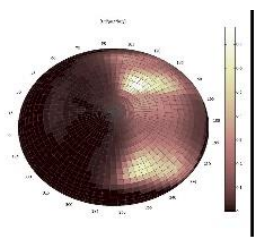

$\mathrm{c}$

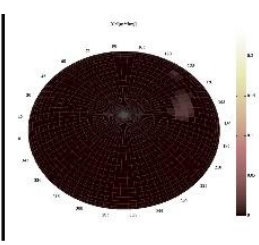

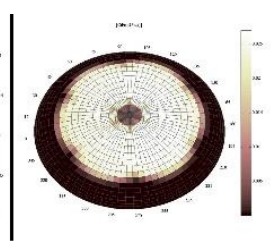

e

Figure 10: Corresponding hemispherical projections of BTDF data of the 5 samples

\footnotetext{
34 International Energy Agency Task 21, Source Book on Daylighting Systems and Components, Chap. 8.3: Optical Characteristics of Daylighting Materials, pp. 8.16 - 8.22, Paris, July 2000.
} 


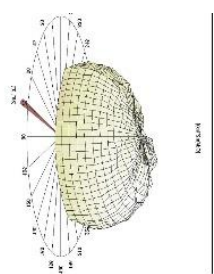

a

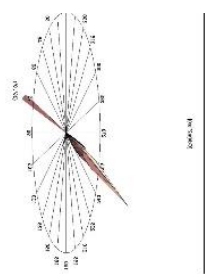

$\mathrm{b}$

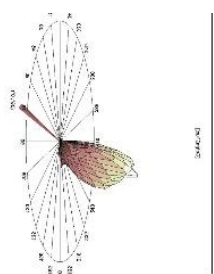

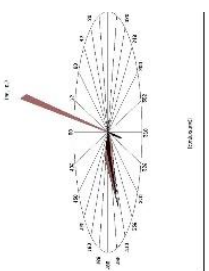

d

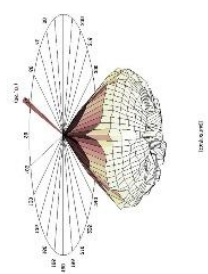

$\mathrm{e}$

Figure 11: Corresponding photometric solids of BTDF data of the 5 samples

\section{Discussion: Pros and Cons}

Based on the CCD imaging technique, the monitoring of BTDF data by the Goniophotometer is relatively time efficient as compared to a scanning Goniophotometer, since the luminance measurement in the exiting hemisphere is realized by several high dynamic range images captured by a camera instead of by a scanning process based on movements of sensors. The maximum incident zenith angle of BTDF measurement of the CCD imaging based Goniophotometer can be larger than that of a scanning Goniophotometer, since the exiting hemisphere is shield by its physical body from the incident hemisphere. Furthermore, the Goniophotometer can monitor samples with relatively large substructures, due to its dimension of light beam and optical path. The BTDF measurement can be independent from ray-tracing simulation software. However, resolution of BTDF data in the exiting hemisphere is limited by the camera pixels, which is commonly lower than that of a scanning Goniophotometer. 


\title{
2.5 Fraunhofer Institute for Building Physics IBP
}

\author{
by Jan de Boer, Yuan Fang; Fraunhofer IBP, Germany
}

Two methods to generate BSDF data are supported: Measurement with a goniophotometer and simulation with a numerical goniophotometer. This is supplemented by an Ulbricht sphere system. The set-up allows to generate BSDF raw data (transmission and reflection) in the two common sampling schemes according to Tregenza and Klems. Data processing then allows to combine the measured BSDFs with different glazing panes to obtain BSDFs, SHGC and other quantities for integrated glazing units (IGUs). Different file formats for import into different design software as DIALux Evo or tools based on the Window7 characterization are supported. A close linkage has been established into the software DIALux Evo. Figure 12 gives an overview of the Fraunhofer IBP activities.

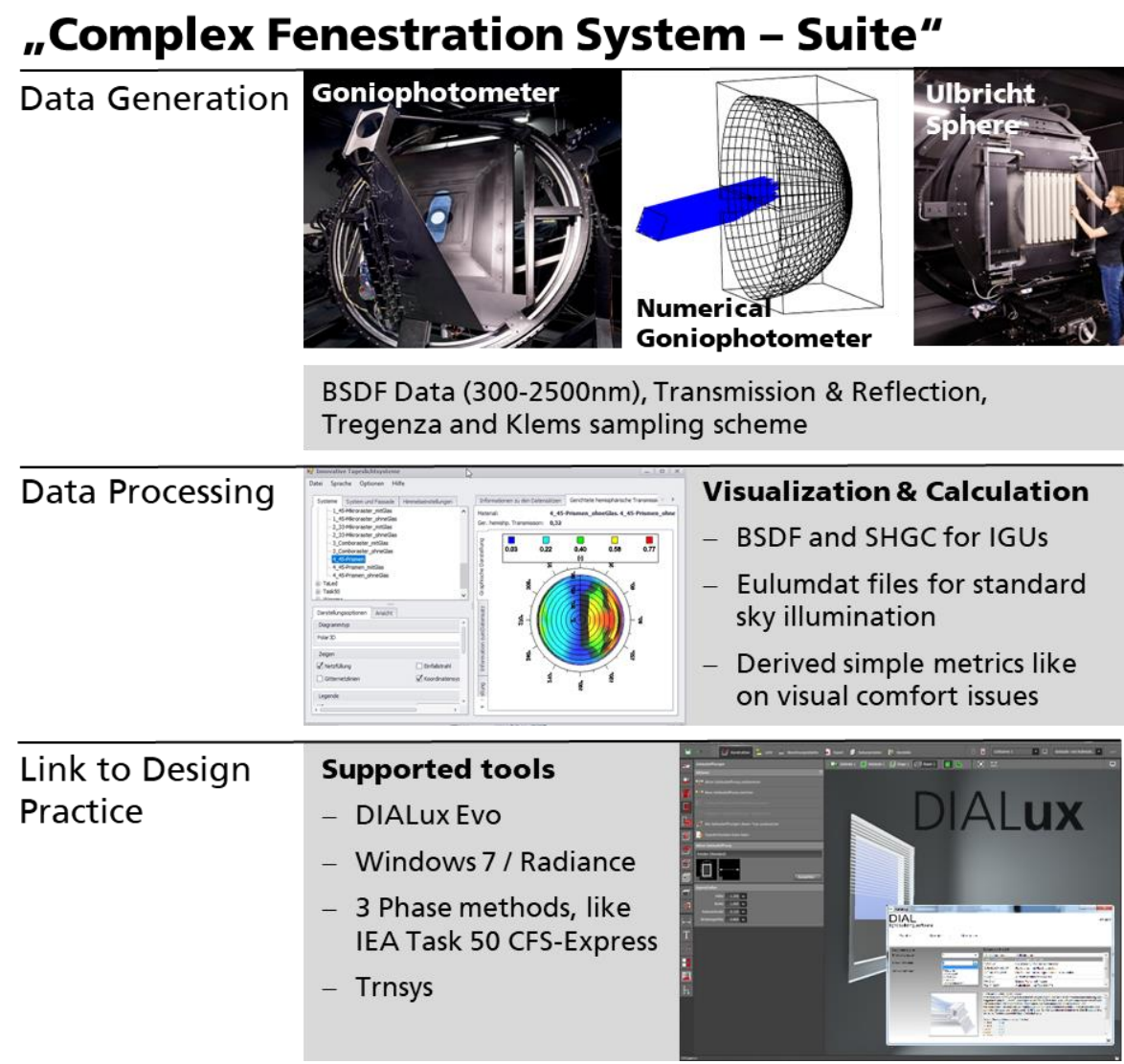

Figure 12: BSDF Assessment and processing at Fraunhofer Institute for Building Physics

\section{Data Generation}

\section{(i) Measurement with a Goniophotometer}

The test device was developed for the measurement of directionally resolved transmission characteristics (BTDF) and reflection characteristics (BRDF) of systems and system surfaces. The height-adjustable and azimuth-adjustable sample holder is illuminated with an HMI tracker. The light transmitted and reflected by the sample falls on a lower and upper screen, respectively. The screens are coated with photometric paint (barium sulphate). They cover $1 / 6$ of the hemisphere above the sample and travel around the sample in six steps to fully capture light transmission and reflection. The luminances on the screens are each recorded by a tracking luminance camera. The testing device is highly automated. It is operated with a control and evaluation software and enables cost-effective measurements. The transmission and reflection characteristics are generally determined for the 145 incident directions according to the Tregenza scheme or according to the Klems scheme. Any other angles are possible. On the exiting side of the BSDF the angular resolution is $2^{\circ}$. Measurement time for light transmission for the 145 incident angles for a static sample, like a light redirecting glass, is in the range of 3 hours. An automatic turning device for slats of venetian blinds, as depicted in Figure 13, allows to assess these systems in a decent measurement time as well. 
Recently, a spectroradiometer has been added to the test stand, such that transmitted and reflected radiation can be recorded in spot measurements in a range between $280 \mathrm{~nm}$ and $2500 \mathrm{~nm}$.

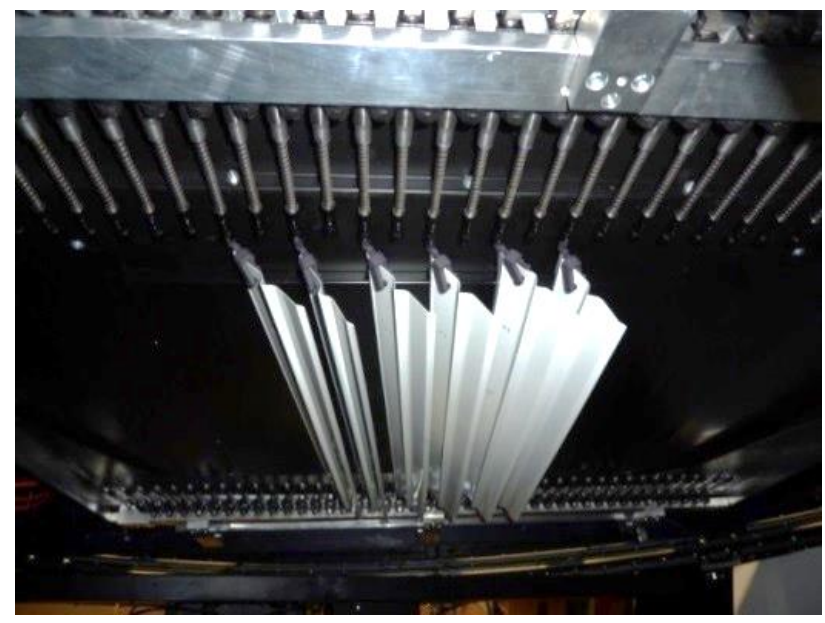

Figure 13: Slat turning device for venetian blind systems on the goniophotometer allowing to vary slat angle and slat distance

(ii) Simulation with a numerical Goniophotometer

Complementary to the measurement device a numerical goniophotometer is available. This can especially be helpful in the design of new systems even if no prototypes exist. Limitations nevertheless lie in the modelling of specific scattering characteristics of real materials (not always a sufficiently accurate numerical reflection or transmission model available for these).

The numerical goniophotometer is based on the commercial raytracing algorithm Zemax. With macros a virtual measurement setup is defined, that allows to perform the calculations based on the Tregenza and Klems sampling schemes with corresponding file output. Calculation times for a complete dataset (all incident angles) are with usually significant accuracy in the range of 10 minutes.

(iii) Ulbricht Sphere

The openable carbon fiber sphere coated with barium sulphate has a diameter of two meters. With a spectroradiometer, the radiation in the range between $280 \mathrm{~nm}$ and $2500 \mathrm{~nm}$ can be recorded in steps of $1 \mathrm{~nm}$. The samples are irradiated with a $10 \mathrm{~kW}$ emitter array. To generate a suitable irradiation spectrum, HMI and halogen lamps were combined. The sphere has two closable ports of $600 \mathrm{~mm}$ diameter each for the transmission and reflection measurement. By means of an automated positioning device both the Ulbricht sphere can be rotated by $180^{\circ}$ and the sample itself in front of the port by $360^{\circ}$. Thus, the sample is rotatable at any height and azimuth angles in the beam path. For movable sun protection devices such as Venetian blinds, a flexibly adjustable motorized turning device for adjusting the slat angle and distance is available on this system as well. The unit is identical to the one on the goniophotometer. These external blind parameters have a significant influence on the g-value. For angle-dependent reflection measurements, specimens can additionally be positioned in the center of the sphere so that they can be rotated by motor using a suspended so-called "center mount system".

The rotatable sample holder is suitable for standard sizes of $600 \times 600 \mathrm{~mm}$ and sample thicknesses up to $100 \mathrm{~mm}$. A separate sample table can also be used to examine larger and heavier specimens with a maximum edge length of $1500 \mathrm{~mm}$, a thickness of $260 \mathrm{~mm}$ and a weight of $300 \mathrm{~kg}$. Special solutions to be sampled, e.g., in facade construction, make such high demands on the test facility.

\section{Data Processing}

The raw data, recorded in the IEA Task 21 Ascii format, can be postprocessed into data files for further use in lighting simulation (xml-based data formats for DIALux or Window7/Radiance use). In this process, it is possible to combine the BSDFs of one component into a layer structure (according to Klems' layer model), allowing to rate integrated glazing units (IGUs). Spectral BSDF (not only v(lambda)) can be further processed in combination with glazing data to calculate solar heat gain coefficients according to ISO 15099. Moreover, the measurement results can be evaluated photometrically and in 
terms of total energy based on or analogous to DIN EN $410^{35}$, DIN EN ISO 52022-3 $3^{36}$ and DIN $14500^{37}$. From the measured data, the parameters for the evaluation of the thermal and visual comfort according to DIN EN $14501^{38}$ can also be determined.

A viewer and calculation software are in use. It allows to view the photometric quantities (directhemispherical transmission, BSDFs) and performs simple calculations like superimposing standard skies at specific locations and time of day / year for different sample orientations and inclines onto the samples BTDFs. This results in candle power distributions, which describe illumination of spaces from the facades for the given sky condition. The distinct distributions can be exported to the Eulumdat format used in lighting simulation software.

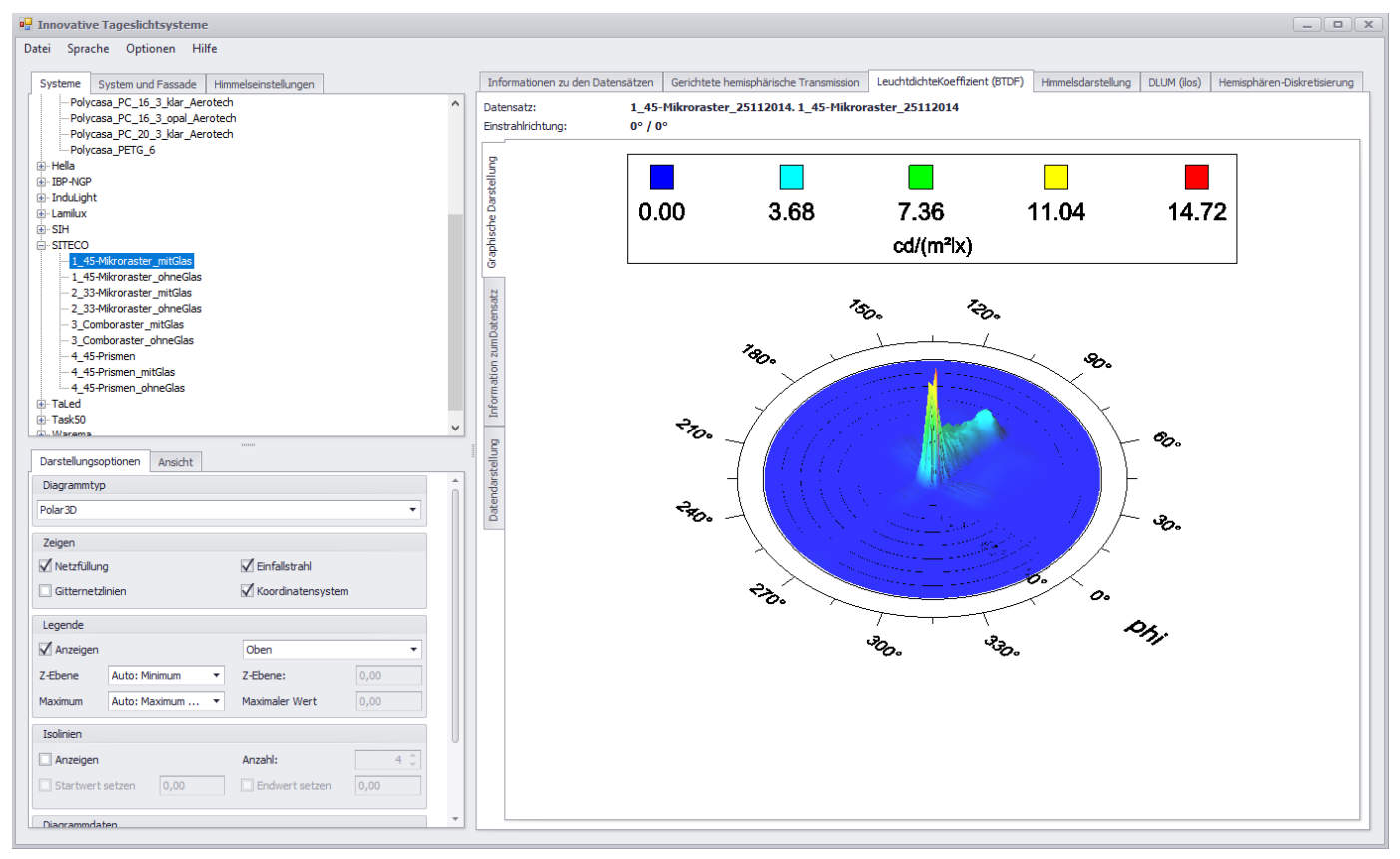

Figure 14: Data viewer

\section{Link to design practice}

The BSDF raw data themselves are only of limited value to building design practice. Therefore, the embedment into lighting software, which puts the BSDF and by this the complex façade system into the building, lighting design and energy context is essential. The data generated with the FHG-IBP equipment can be used by practitioners in the tools DIALux Evo or Radiance and can be included into simple tools based on the 3-phase method. In addition, linkage for thermal, energetic modelling with angular dependent SHGCs is provided.

An especially close linkage has been established with the software DIALux Evo. An XML based file format can - aside the static BSDF - hold other product specifications (meta data). In the case of venetian blinds for instance control curves, to operate the louvres in a "cut-off" mode can be specified. Orientation sensitive components as sun protection gratings for rooflights are enriched with information, which allow the correct placement into the roof structure directly by the software DIALux Evo.

Within the software DIALux CFS handling is embedded into a graphical user interface. This allows to configure the glazing, sun- \& glare protection units (cf. Figure 15 and Figure 16) or the rooflight elements. Sun- and glare protection systems and rooflights are assessed with plugins via drag and drop, managed and provided by the specific manufacturers. Data of more than 50 façade components are available.

35 DIN EN 410: Glass in building - Determination of luminous and solar characteristics of glazing, 2011.

36 DIN EN ISO 52022-3: Energy performance of buildings - Thermal, solar and daylight properties of building components and elements - Part 3: Detailed calculation method of the solar and daylight characteristics for solar protection devices combined with glazing, 2018.

37 DIN 14500: Blinds and shutters - Thermal and visual comfort - Test and calculation methods, 2021.

38 DIN EN 14051: Blinds and shutters - Thermal and visual comfort - Performance characteristics and classification, 2021. 
Product data of the façade components used in a project may automatically be included in the project documentation.
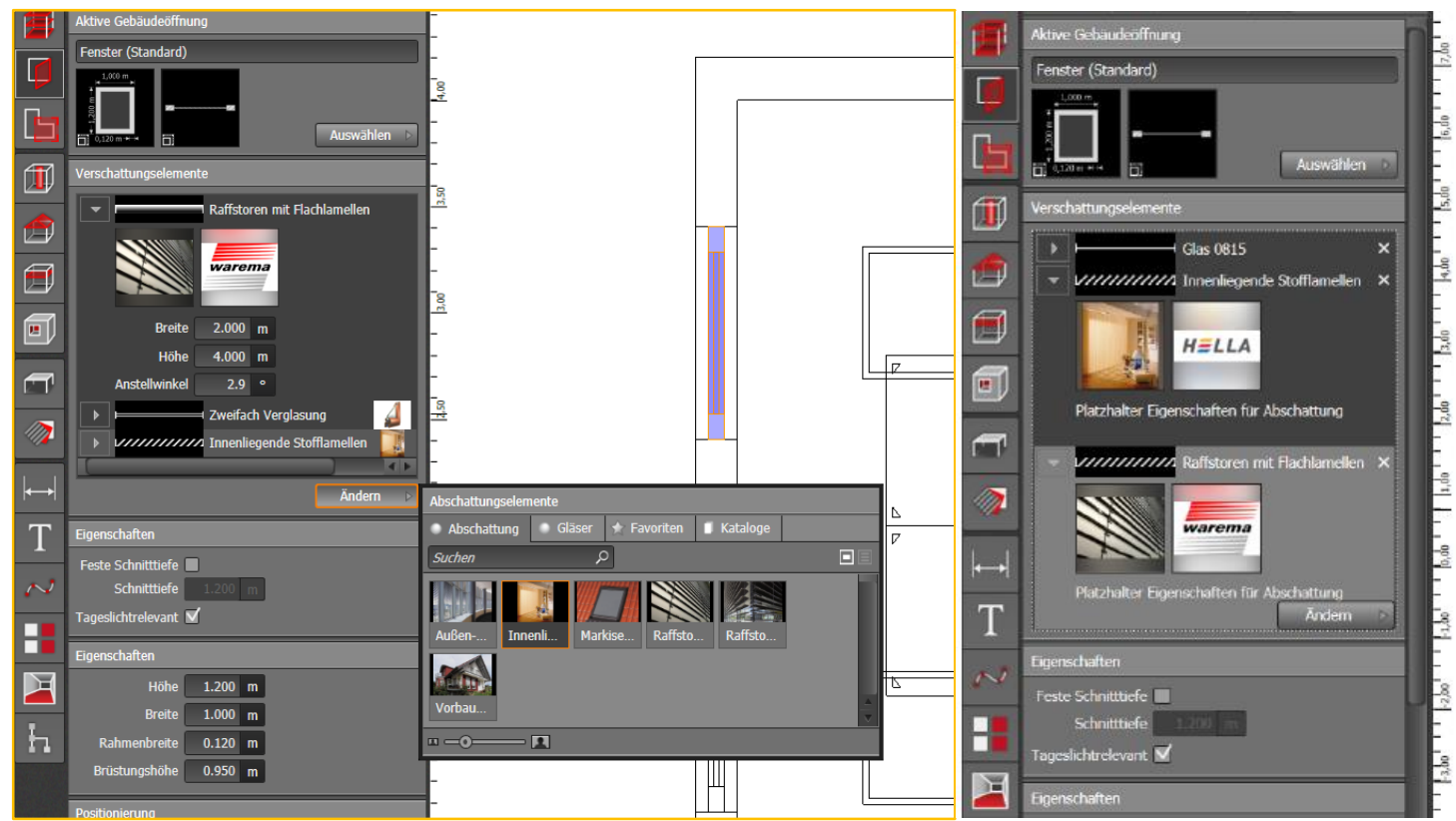

Figure 15: Component selection (left) and definition of layer model (right) in DIALux Evo.
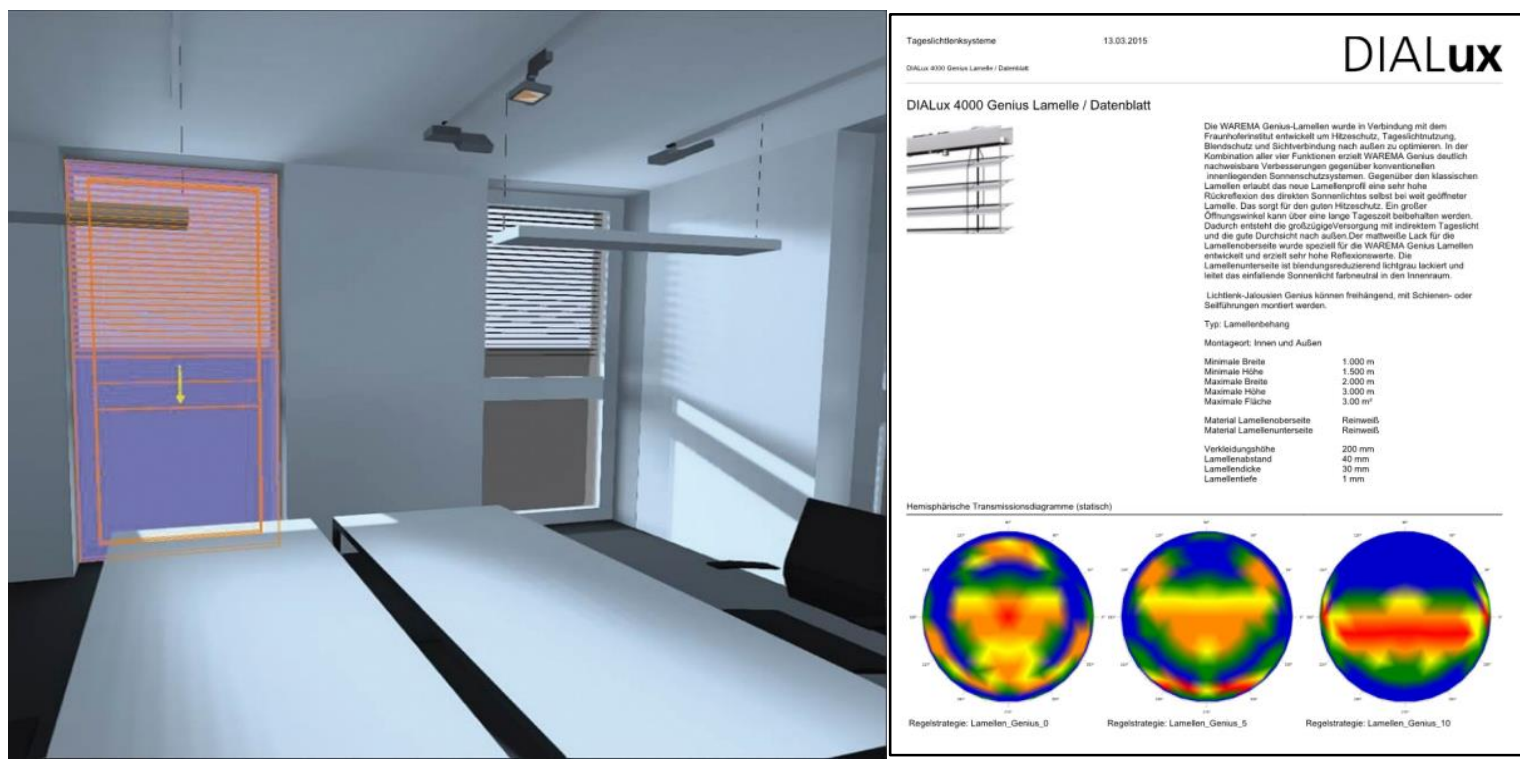

Figure 16: DIALux Evo simulation of an office space with venetian blinds based on BSDF measurements in the goniophotometer at FHG-IBP (left). Product documentation (right).

\section{Discussion: Pros and Cons}

Regarding BTDF generation the measurement principle based on CCD imaging technique combined with an automated sampling positioning system may be considered advantageous as it allows rather fast BTDF assessments. By this data sets at reasonable costs for static as well as adjustable samples like blinds with different slat inclines can be provided. A drawback of this approach in comparison to scanning photometers is the limitation of the resolution by camera pixels. Scanning goniophotometers may here deliver higher resolution BTDFs, possibly better suited for (future) glare analysis. The 
numerical BTDF generation has proven to be helpful in the development process of new optical components for façade applications. It allows quick design alterations without having to build physical models of the new optical structures.

The Ulbricht-Sphere can be used for complementary flux measurements, which can be obtained from the goniophotometer by integration as well. Especially at FHG-IBP it allows to assess the spectral flux of bigger and thicker samples than the goniophotometer with a relatively small aperture and angular limitations in assessing thicker samples

With regard to practical application and use of BTDFs a full dataflow from the measurement device directly into selected lighting design tools is provided. Numerically different analysis operations relating to different standards can be performed on the data. Future improvements can be the provision of a bigger number of datasets of façade elements (by better promotion by the manufacturers) and an extension of the capabilities of the tools employing BTDF data. The later addresses for instance the introduction of annual calculation schemes relying on the recorded BTDF data ( 3 or 5 phase method) in the widely used software DIALux Evo. 


\title{
2.6 Belgian Building Research Institute BBRI
}

\author{
by Bertrand Deroisy, BBRI, Belgium
}

Using Bidirectional Scattering Distribution Functions (BSDF) is a way to characterize the scattering properties of surfaces and materials. A BSDF function describes the radiance/luminance of scattered light as a function of the angle of outgoing light from the surface normal $\left(\theta_{s}\right)$ and as a function of the angle of incidence of the light illuminating the surface $\left(\theta_{i}\right)$. The BSDF function for a specific sample varies depending on the following parameters: Angle of incidence (AOI), wavelength, refractive index, plane of incidence and direction of incidence (front/back). In most cases the angle of incidence is the most critical parameter. By default, BSDF-data should only be measured at typical angles of incidence, for example $10^{\circ}, 30^{\circ}, 50^{\circ}, 70^{\circ}$. Only special surfaces and materials will require more measurements at specific angles of incidence.

At BBRI the approach (for the PROSOLIS project) is to precisely assess the scattering properties by measurements on a set of representative samples and to investigate the impact of this measured BSDF data compared with simple models such as specular reflectance models and isotropic or Lambertian diffusers. More complex BSDF models including angular variations of the optical properties were also tested.

The general workflow for the generation of BSDF is a combined measurement and simulation process which consists of three steps:

1. Characterization of materials and surfaces

2. Building geometrical model of daylighting system

3. Running simulation using imported BSDF data

\section{Step 1: Characterization materials and surfaces}

Measurement of BSDF-properties using the Reflet 180 equipment or using the BSDF library if the material has already been characterized. The measurement also includes the directional-hemispherical transmittance/reflectance at different angles with an integrating sphere (according to standard EN 14500). Indeed, the BSDF measurement only provides for the general profile of the BSDF function, while the direct-hemispherical data provides the total value of reflectance or transmittance to be used to calibrate each BSDF function to the correct integrated value.

For solar shading screens the BSDF data was measured in transmittance at angles of $0^{\circ}$ (normal incidence) and then by steps of $10^{\circ}$ up to $70^{\circ}$. As the reflection is more regular on this type of products the BSDF data in reflection were only measured at near-normal incidence $\left(8^{\circ}\right)$ and at an angle of incidence of $40^{\circ}$.

For the metallic slats of typical venetian blinds, the BSDF measurements were done in reflectance only at incidence angles of $10^{\circ}, 30^{\circ}, 50^{\circ}$ and $70^{\circ}$. For some surfaces measuring properties at grazing angles (around $80^{\circ}$ ) would however be interesting because properties can vary a lot.

\section{Step 2: Building a geometrical model of the daylighting system}

The LightTools ${ }^{39}$ software allows to introduce a geometrical description of a model or to import it from other CAD applications, more particularly the Solidworks software environment. Simple geometrical models can directly be modelled in the optical calculation software. Modelling unusually shaped optical components or geometrically complex elements is also possible in LightTools, as a flexible 3D solid modeling design tool is available.

The sky is modelled as a large hemisphere. Any luminance distribution from the sky can be modelled by setting the luminance of each point of the hemisphere at an appropriate value. For daylighting simulations, a sky model with a $5^{\circ}$ resolution in solar altitude and a $10^{\circ}$ resolution in azimuth was used.

39 https://www.synopsys.com/optical-solutions/lighttools.html, (accessed 05 February 2019). 
For all intermediate positions an interpolation is done so that a continuous sky model is obtained (Figure 17). The ground surface is modelled as a perfect horizontal plane with diffusing properties. Other geometries and reflectance properties for the ground surface are possible.
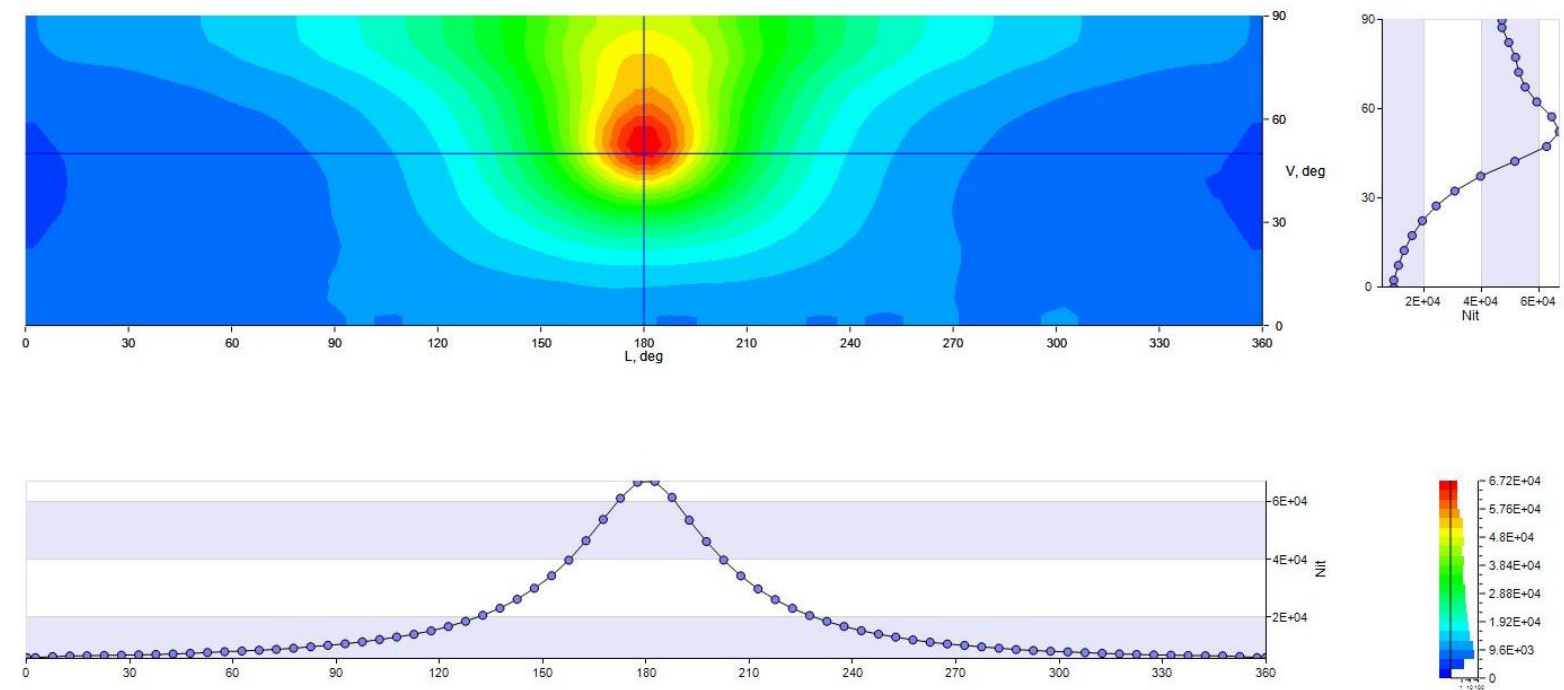

Figure 17 : CIE Clear sky (Type 12) with solar altitude at $40^{\circ}$

\section{Step 3: Running simulations using imported BSDF data}

Advanced software applications can model scattering using BSDF data with distributions determined at multiple angles of incidence. Complex interpolation rules are therefore implemented to reconstruct an BSDF function for each incident radiation. In LightTools this capability supports line scan (1D slice) data, anisotropic slice scans (two 1D slices), and full scan (2D grid) data obtained from measurement suppliers. Even if line scans are provided for various angles these are converted to 2D isotropic data when running simulations. Instead of using measured BSDF data it is also possible using analytic data and predefined BSDF models.

For each surface four files are supplied to import the data in the LightTools software:

- Master file (file coupling the informations)

- RT file (file giving the total hemispherical reflectance and transmission)

- bsd file (file including the scatter data)

- OPR file (used to load the data)

An interface to visualize the BSDF data is integrated in the LightTools software. Transparent materials are considered on basis of their basic physical properties, refractive index and extinction coefficient, and with their spectral variations if required.

Simulations are then run using, in priority, the forward raytracing engine. The number of rays to be traced depends on the required accuracy and the complexity of the scene. Following a sensitivity study, for the conditions of the PROSOLIS project approximately 50 million rays are necessary to correctly assess multiple reflections that can occur when light passes through a façade system with a solar shading device.

If the simulations intent is to determine quantitatively the daylight supply through a façade system a vertical control plane ('receiver') was placed just behind the envelope system. Total light fluxes were generated by simulations integrating BSDF data measured for the materials used as solar shading devices. For each orientation, the daylight supply is calculated for a cumulated clear summer (15 June) and winter (15 December) sky. This daylight supply therefore represents the total light flow through the 
combination of glass with the solar shading device fully extended for a representative day with perfectly clear sky.

If the simulation intent is to estimate the risk of glare in a space the light distribution inside matters more than the light flux. In particular the redirection of light when passing through the system as well as the multiple diffuse interreflections are critical. Glare is a peak moment evaluation, which means that we have to identify precisely the moments when glare could occur. Simulation for glare assessment purposes are done at specific moments. As accurate luminance distributions in the field of view of an observer are useful hybrid raytracing method (combination of forward and backward raytracing) with a higher number of rays should be used.

\section{Discussion: Pros and Cons}

The main advantage of this procedure is that BSDF measurement results, whatever their resolution, can be used directly in the simulations. There is no need for intermediate steps, realizing a BSDF matrix for an equivalent layer for example, as the system with its real geometry and detailed material properties are modelled. A distinction could thus be made between first-order and second-order BSDF approaches. The current approach would be a first order BSDF evaluation as the BSDF data of the surfaces are directly used. The multiple steps in the calculation process using a second-order BSDF evaluation introduce inaccuracies and potential errors.

One disadvantage of this approach is that simulations for a whole year with reduced time-steps are highly time-consuming. However, a climate file for daylight is only a random distribution of sky conditions for one place. An interesting path to be explored for glare assessments would be to elaborate a first evaluation sorting out the critical and representative cases, and then realize accurate simulations for these selected moments. 


\subsection{Bartenbach}

\section{by David Geisler-Moroder, Bartenbach, Austria}

The general workflow for the generation of BSDF is a combined measurement and simulation process which consists of (up to) five steps:

1. Characterization of base material

2. Set-up of geometrical model of daylighting system

3. Simulation of BSDF data

4. Measurement of direct-hemispherical transmittance

5. Validation / calibration of BSDF data against transmittance values

\section{Step 1: Characterization of base material}

The material characterization is usually provided by the manufacturer either as BRDF/BTDF data or as parameters for parametric models such as ABg or Gaussian (e.g., by Alanod ${ }^{40}$ ). For refractive systems, the index of refraction, absorption coefficients and - if relevant - parameters for volume scatter are requested.

If the necessary data cannot be provided by the manufacturer, the material properties are measured using goniophotometer devices. The resulting data are fitted to parametric models for further use in the simulation tools LucidShape or Radiance.

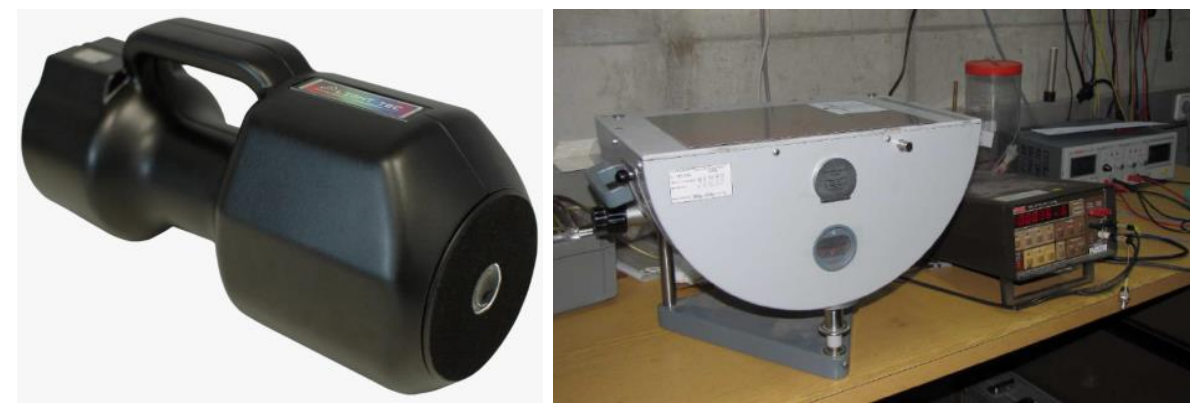

Figure 18: The Light Tec Mini-Diff V2 and a Carl Zeiss in-plane measurement goniometer for the characterization of base materials

\section{Step 2: Set-up of geometrical model of daylighting system}

A geometrical model of the daylighting system is either provided by the manufacturer or built in a CAD system and prepared for the import in the simulation software. If a final BSDF including a glazing unit shall be produced, the glazing is also included in this 3D model. Usually, the geometric model is set up large enough to be able to extract a representative area of the system in the center. In this way edge effects can be avoided and a generic BSDF for the system can be generated.

\section{Step 3: Simulation of BSDF data}

Depending on the desired output and the required functionality

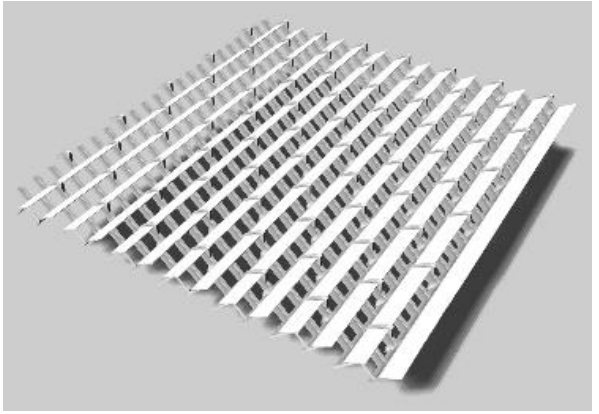

Figure 19: CAD model of complex fenestration system (e.g., use of provided material BRDF data), the system geometry is imported and simulated either with the forward raytracing software packages LucidShape or ASAP or with the backwards raytracer Radiance. As for LucidShape and ASAP no ready-to-use tools to simulate BSDFs (i.e., generate illumination and split up sensor readings for given directions / patches) exist, these functions had to be implemented. In Radiance, the genBSDF tool can be used which allows to

40 https://www.alanod.com/en/ 
generate ready-to-use XML BSDF files with data resolution either according to the WINDOW 7 specification (Klems' resolution) or variable resolutions using tensor tree data structures. Other discretizations would have to be implemented by the user via a cal-file.

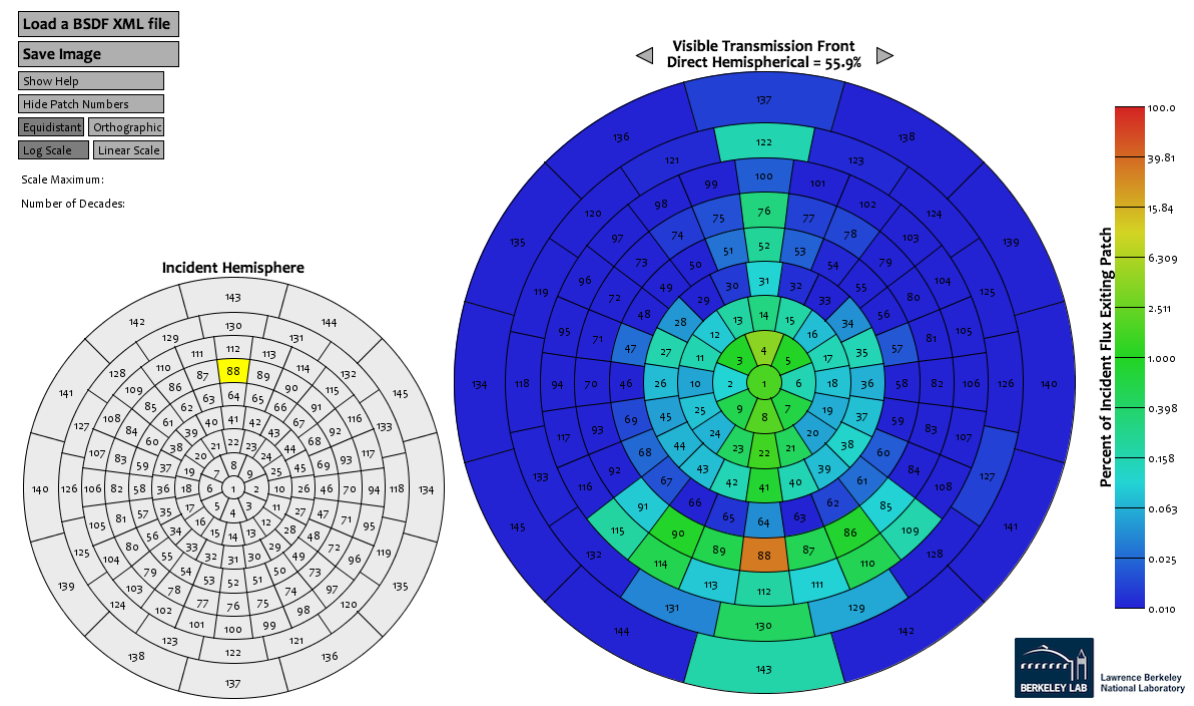

Figure 20: Falsecolor representation of BSDF values

\section{Step 4: Measurement of direct-hemispherical transmittance}

A sample of the daylighting system is used to measure angular dependent direct-hemispherical transmittance values. Using the sun (outdoors or indoors via redirecting mirrors) or a nearly parallel artificial light beam ("artificial sun") and an integrating Ulbricht sphere, these values are measured in the lighting laboratory. This transmittance measurement is used in Step 5 to validate the simulated BSDF.
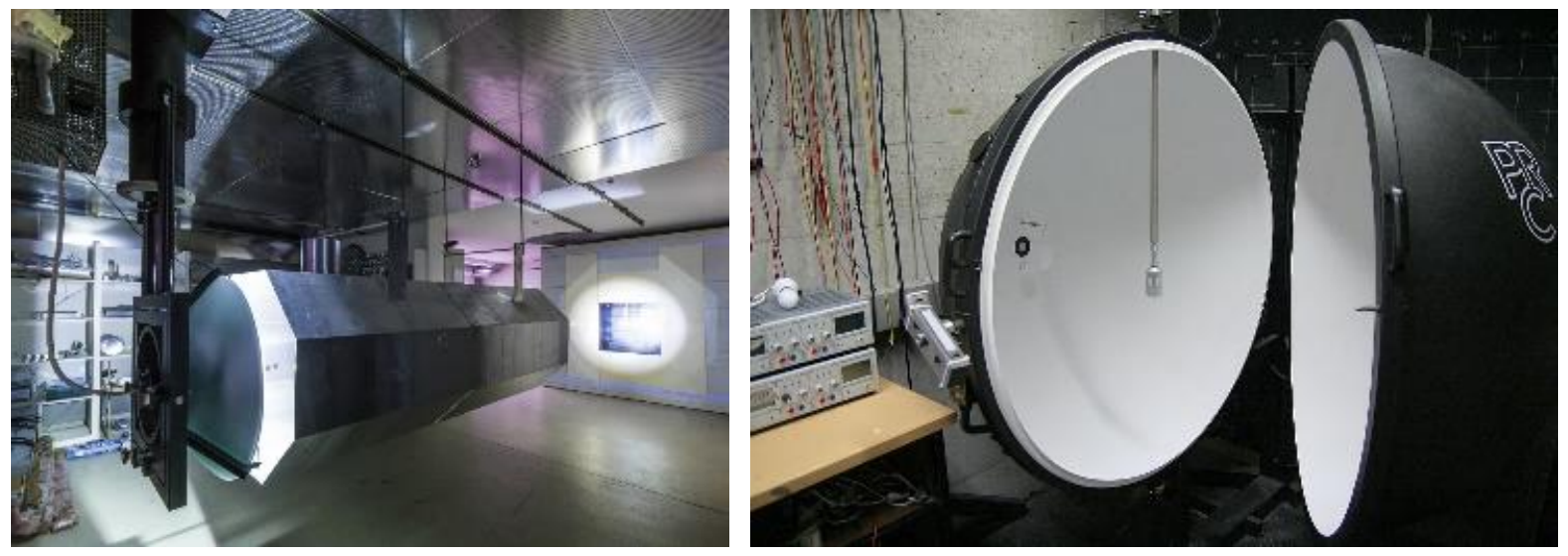

Figure 21: Devices to measure direct-hemispherical transmittance: artificial sun (left) and integrating sphere (right). 


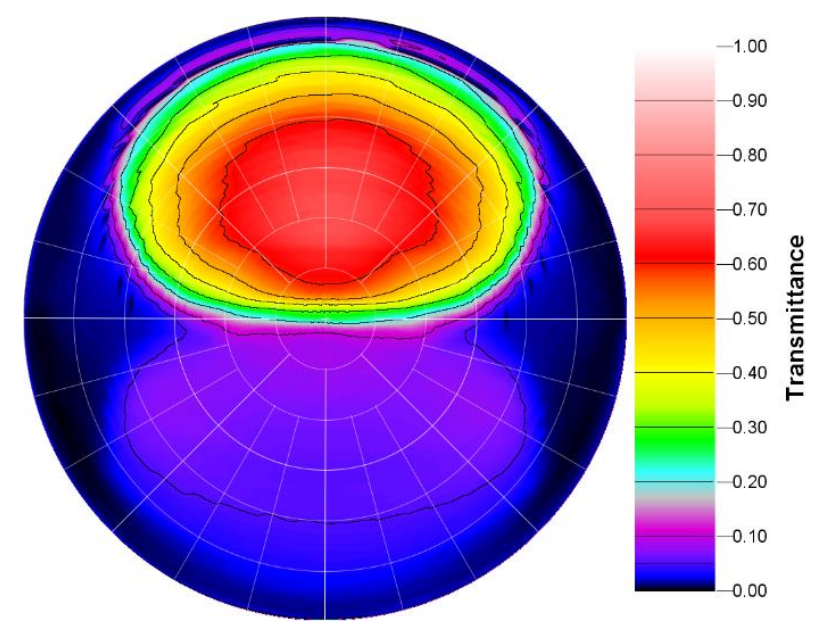

Figure 22: Falsecolor representation of angular-dependent, direct-hemispherical transmittance

\section{Step 5: Validation / calibration of BTDF data against transmittance values}

The BTDF obtained from the simulations in Step 3 are integrated to obtain direct-hemispherical transmittance values. As these values correspond to the overall luminous flux passing through the daylighting system, it is seen as the main criterion to get correct. In this step the measured values from Step 4 are used as reference and the simulated BTDF values are calibrated to match the integrated transmittance.
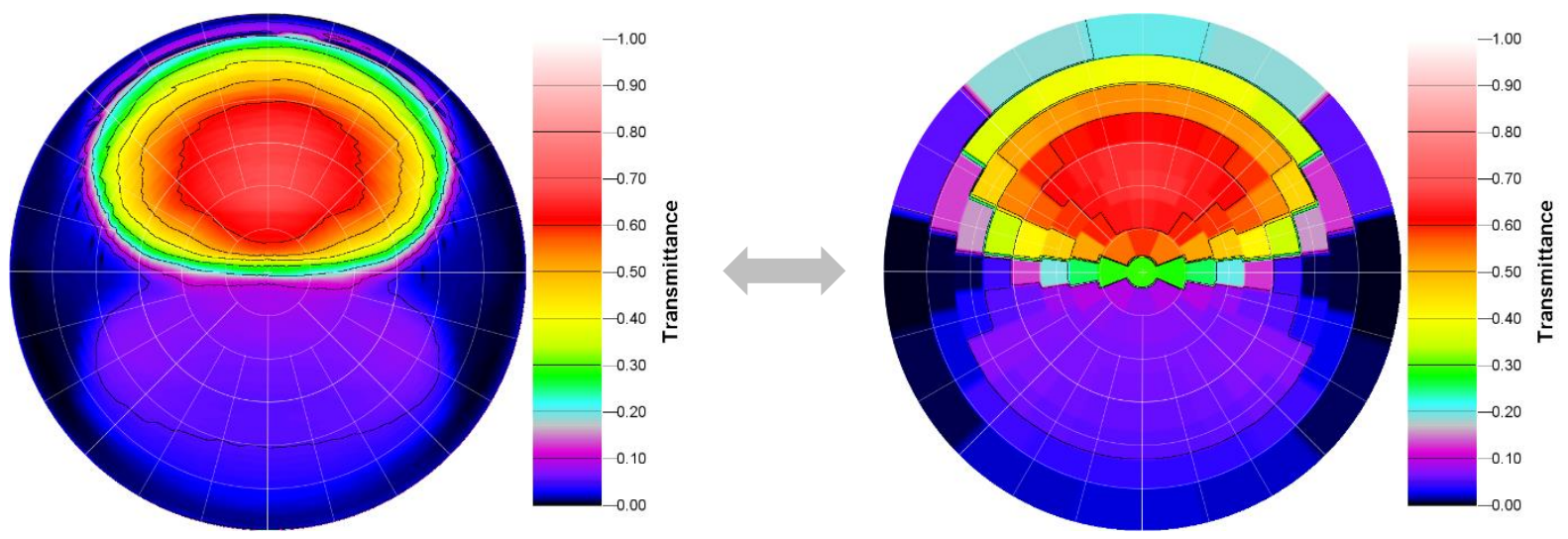

Figure 23: Comparison of angular-dependent, direct-hemispherical transmittance from measurement and derived from BSDF data

\section{Discussion: Pros and Cons}

Both available measurement devices for the characterization of the base material are limited to some extent. The Carl Zeiss in-plane measurement goniometer is only suited for isotropic materials, the Light Tec Mini-Diff V2 can handle anisotropic materials, but only provides four polar angles for each azimuthal angle of incidence. For complex, anisotropic base materials where the manufacturer does not provide an adequate data set or analytic description, the characterization is ordered externally.

The geometrical model of the daylighting system usually represents the theoretical, developed structure, but does not include manufacturing tolerances. However, experience shows that modern manufacturing processes are precise enough so that the simulated BSDFs do not differ significantly from the real light scattering properties.

The comparison and calibration of the BTDF data set to the additional measurement of a full set of direct-hemispherical transmittance values is a major benefit of this workflow. With this step the accuracy of the overall transmitted flux is guaranteed and thus the energy balance correctly represented. 


\title{
2.8 High Performance Building Lab, Hunan University
}

\author{
by Zhen Tian, School of Architecture, Hunan University, Changsha, China
}

In the High Performance Building Lab at the School of Architecture, Hunan University, ongoing research have been conducted on the methods of modeling Complex Fenestration Systems (CFS) for daylight and thermal, especially for the Prism Daylighting Redirection Fenestrations (PDRF). The research team works with manufacturers to find ways to model the PDRF quickly and precisely in practice, through combined simulations and measurements.

As different manufacturers may produce specific shape, size, and configuration of prismatic film products for daylighting and/or solar shading, the direct-hemispherical transmittance scattering properties of different prismatic film products need to be analyzed. Meanwhile, the actual micro-structure and shape of a prismatic film produced may be different from the original product design in the manufacturing process.

\section{Data generating process}

To improve the modeling process and to avoid possible errors, the workflow for the generation of BSDF $\mathrm{XML}$ files is as follows:

1. Measurement of the micro-structure profile of the prismatic film with a 3D laser microscope to find out the actual structure, angles, and dimensions of the micro-structures.

2. Description and setup of the geometric micro-structure model of the prismatic film in detail with the Rhino+Grasshopper program.

3. Generation of the BSDF XML file of the prismatic film with the genBSDF program in Radiance.

4. Validation of the generated BSDF XML file with goniophotometer measured results.

Figure 24 presents the micro-structure of one type of prismatic film for daylight redirecting under a 3D microscope scanner. The corresponding prismatic film micro-structure section profile modeled with Rhino+Grasshopper is presented in Figure 25.

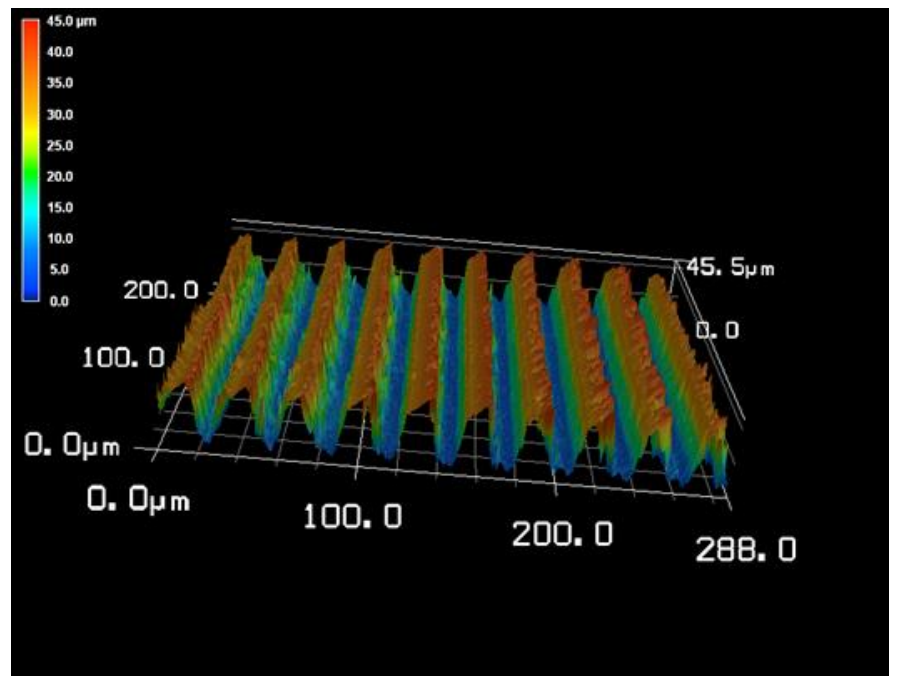

Figure 24: 3D laser microscope scanning of the micro-structure of one type of prismatic film.

Using the genBSDF function within Radiance, the BSDF XML file can be generated with the detailed geometric information from Grasshopper. The luminous flux distribution generated from genBSDF can be reviewed using the BSDFViewer developed by the Lawrence Berkeley National Laboratory (Figure 26). The genBSDF XML file in this research is also validated using goniophotometer measured results from Lawrence Berkeley National Laboratory. 


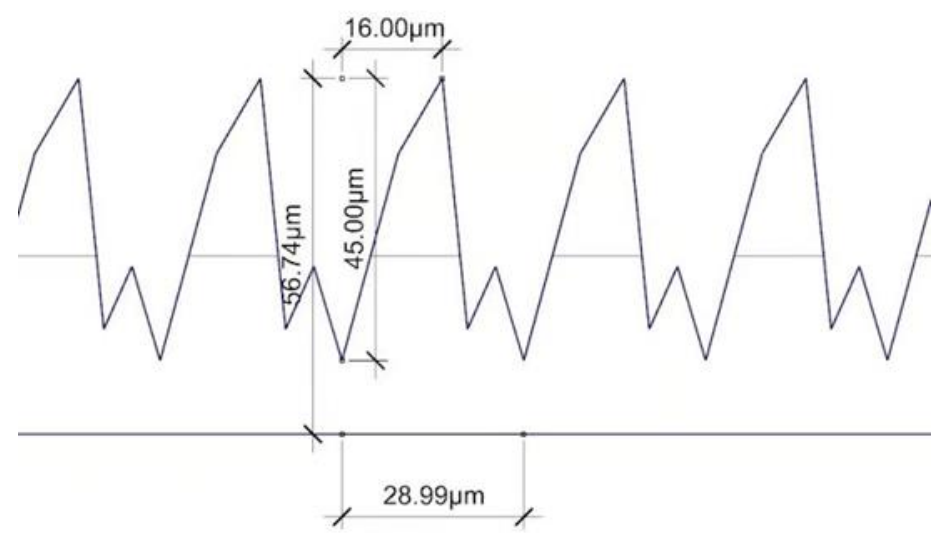

Figure 25: Modeled prismatic film section profile in Rhino+Grasshopper.
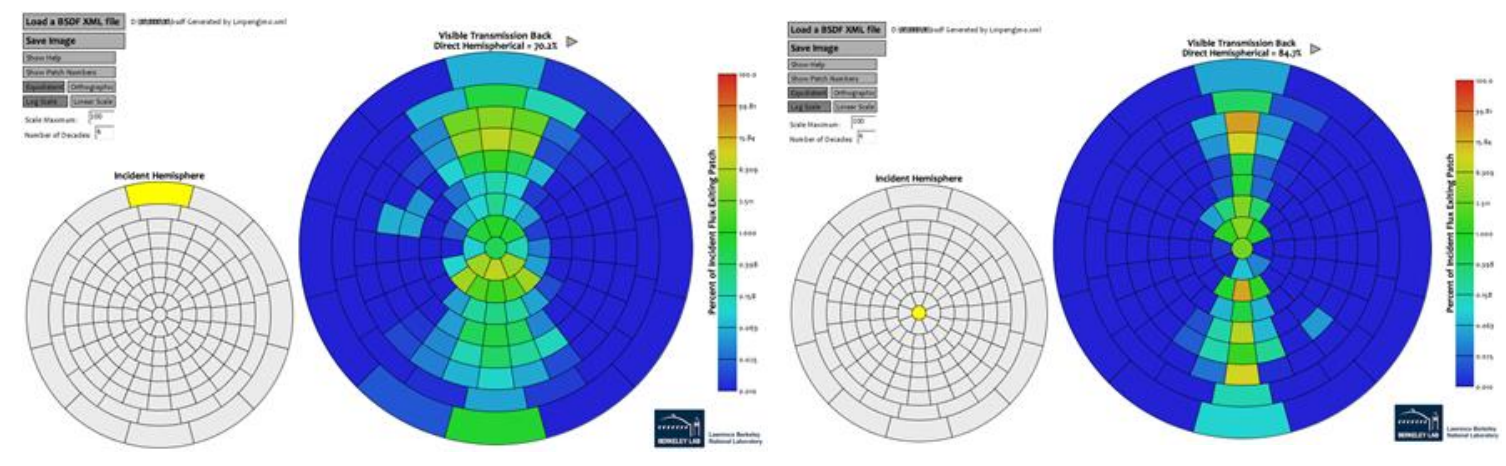

Figure 26: Modeled prismatic film falsecolor representation of BSDF values.

\section{Discussion: Pros and Cons}

Compared to the general practice of generating BSDF XML files with genBSDF or other programs, this procedure adds an additional process of using 3D laser microscope scanner to obtain the detailed section geometric data. This 3D scanning is an easy and fast process, improving the modeling detail which may have important impact on the prismatic film direct-hemispherical transmission properties.

This additional process provides a method to improve the modeling precision. However, not every CFS can be scanned using a 3D laser microscope. Meanwhile, the BSDF XML file generated may be different from the goniophotometer measurement results and a thorough check is strongly suggested from our past experiences. The geometric profile modeling with Rhino+Grasshopper provides a useful parametric description of the CFS and can be incorporated as a standard process when modeling CFS in practice.

\section{Validation using HDR photography and BSDF modeling}

Research has been conducted by the High Performance Lab at Hunan University to compare the difference using HDR photos and BSDF modeling of Prism Daylighting Redirecting Fenestrations.

A Canon EOS 5D Mark II camera with an EF 8-15 mm fisheye lens was placed at positions close to subjects' eyes and used to take a series of low dynamic range (LDR) photos with different exposures. Each series included 9 shutter speeds: 1/15", 1/30", 1/60", 1/125", 1/250", 1/500", 1/1000", 1/2000", and $1 / 4000$ ", with f-stop constant at 5.0. A calibrated target was marked in the field of view and a Konica Minolta luminance meter (LS-160) was put beside to measure the luminance at the reference point. The vertical eye illuminance was measured with a Konica Minolta illuminance meter (T-10A) at each viewing position of the camera, which was placed close to the subjects' eye level while sitting, facing the direction observed by the subject in that particular position (Figure 27). In total, 123 cases were measured and simulated. 


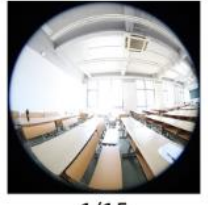

$1 / 15$

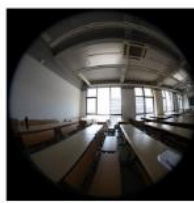

$1 / 125$

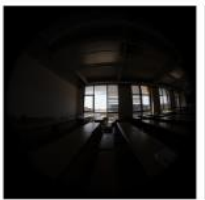

$1 / 1000$

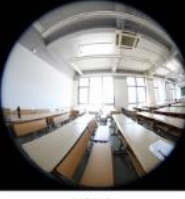

$1 / 32$

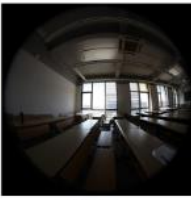

$1 / 250$

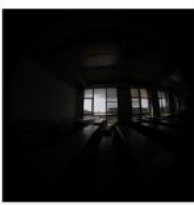

$1 / 2000$

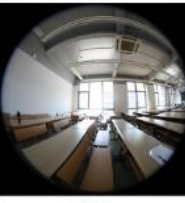

$1 / 64$

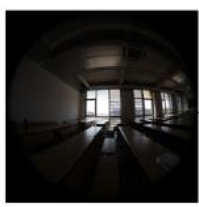

$1 / 500$

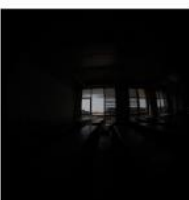

$1 / 4000$
1.Merging of LDR

images in Photosphere

2.Vignetting correction

3.Photometric adjustment

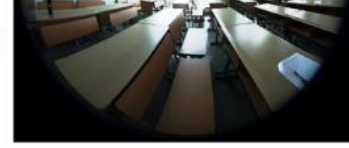

HDR photograph

Figure 27: HDR photograph generation and calibration process

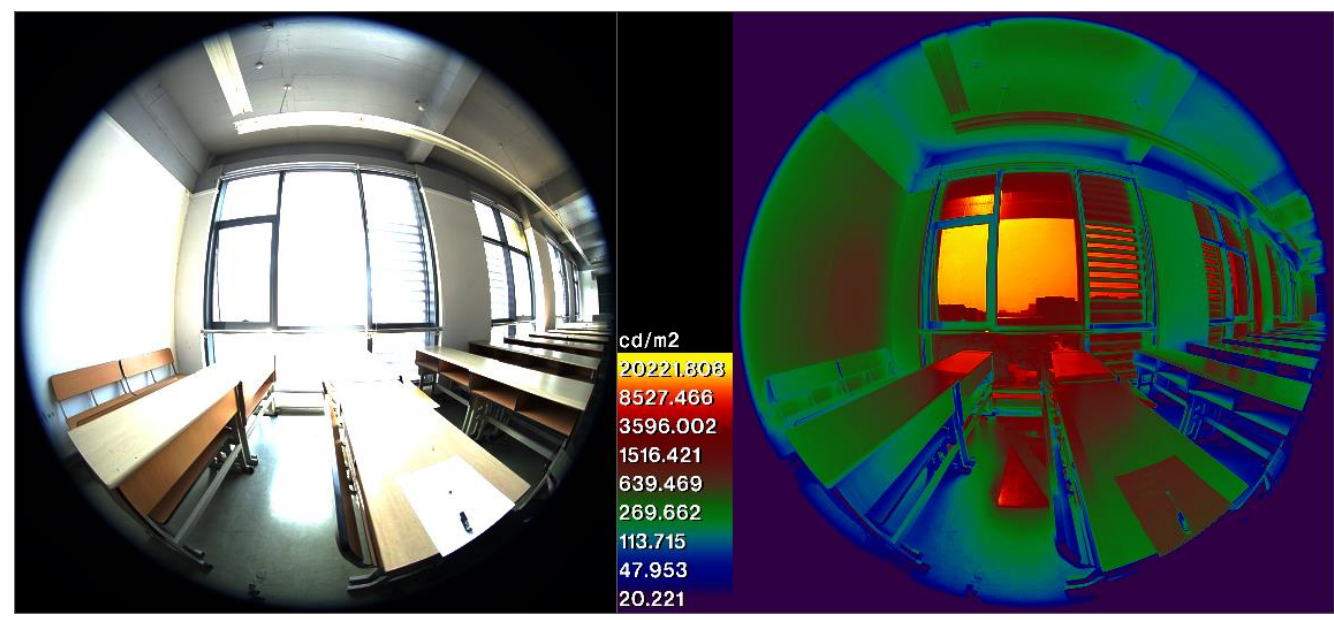

Figure 28: Luminance mapping derived from HDR photograph

The generated HDR photographs then were checked before using for luminance mapping (Figure 28). The evalglare program was employed to calculate the vertical eye illuminance (Ev) values and compared to the values measured with the illuminance meter (T-10A). If the relative error was no larger than $25 \%{ }^{41}$, the quality of the generated HDR was acceptable (Figure 29).

41 Pierson, C., Cauwerts, C., Bodart, M., \& Wienold, J. (2021). Tutorial: Luminance Maps for Daylighting Studies from High Dynamic Range Photography. LEUKOS - Journal of Illuminating Engineering Society of North America, 17(2), 140-169. 


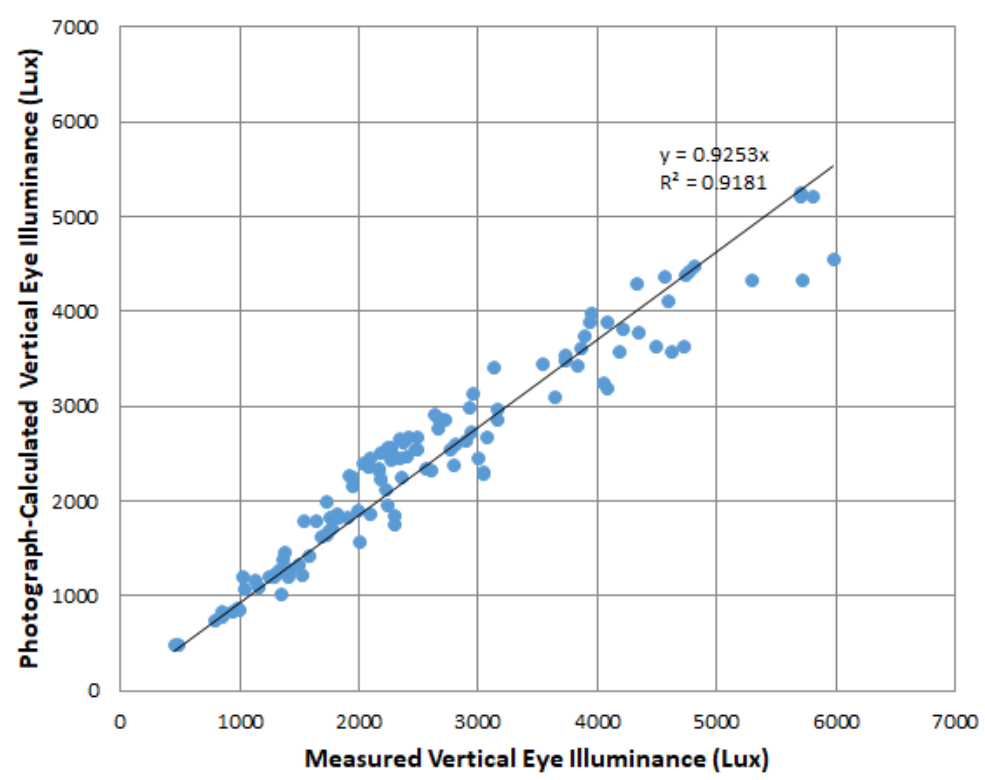

Figure 29: Measured and photograph-calculated vertical eye illuminances

Radiance was applied to conduct daylighting simulations with PDRF in the study. To get accurate realtime sky illuminance for use, a micro weather station was set up on a close nearby building roof to measure the global horizontal irradiance, direct irradiance and diffuse irradiance. The measured direct normal and horizontal diffuse irradiance values were input into the Perez sky model to generate sky distributions. Then image-based simulation with Klems BSDF data sets was employed to produce simulated images in Radiance, and discomfort glare indices of DGI and DGP were calculated with evalglare.

Figure 30 presents the luminance results comparison of HDR photograph and simulated image with Klems BSDF data and real time weather data. The correlation analysis in Figure 31 shows a good agreement between BSDF simulated DGP and Photograph-calculated DGP values $\left(R^{2}=0.8842\right)$, but in Figure 32 a weak correlation is presented between simulated DGI and Photograph-calculated DGI $\left(R^{2}=0.4764\right)$. It can be observed that the agreement between BSDF simulated DGP and Photographcalculated DGP is better than those for DGI calculations.

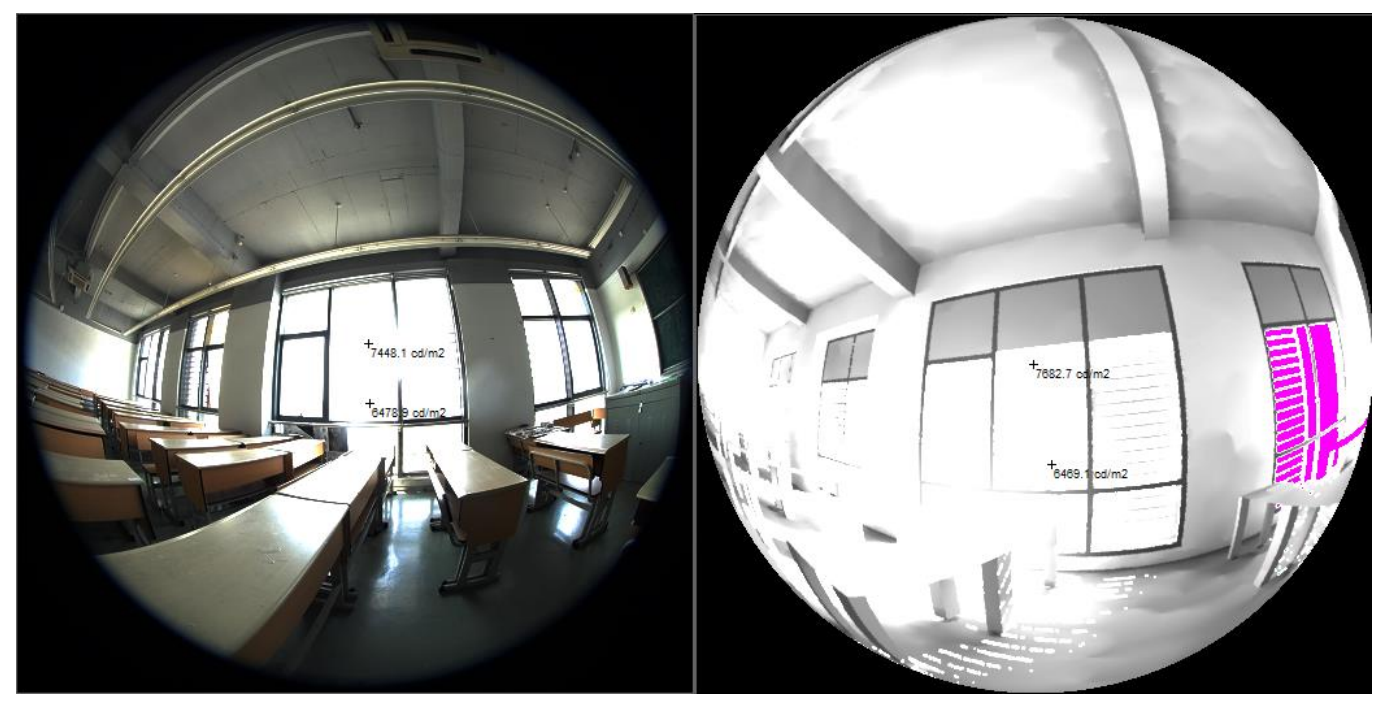

Figure 30: HDR photograph (left) and corresponding Klems BSDF simulation (right) 


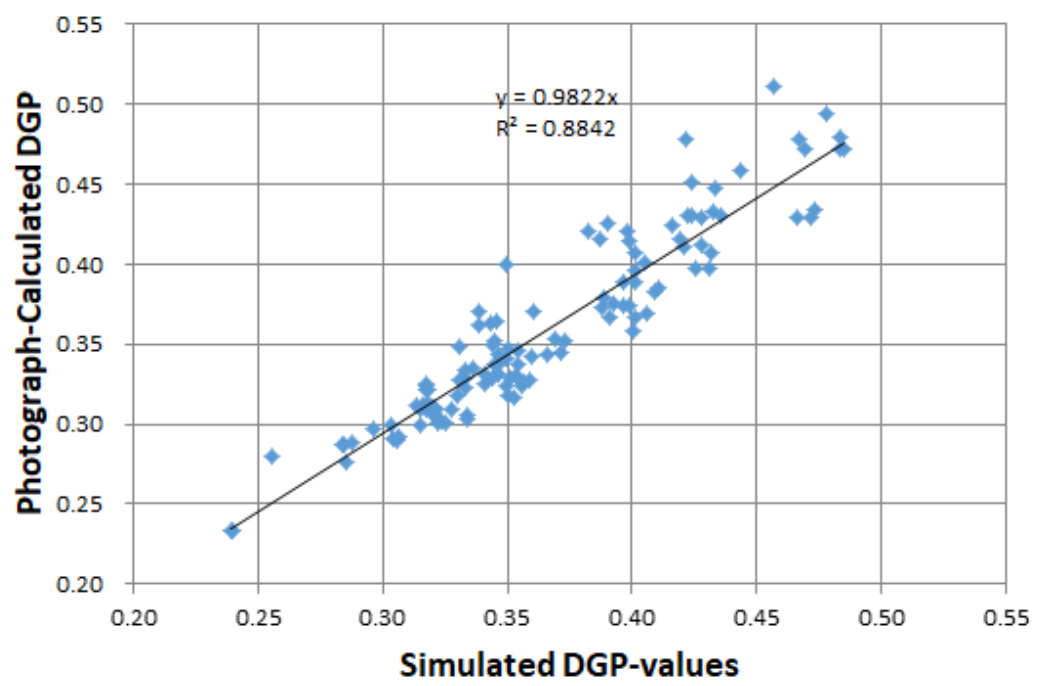

Figure 31: Comparison of simulated DGP-values with Photograph-calculated DGP-values

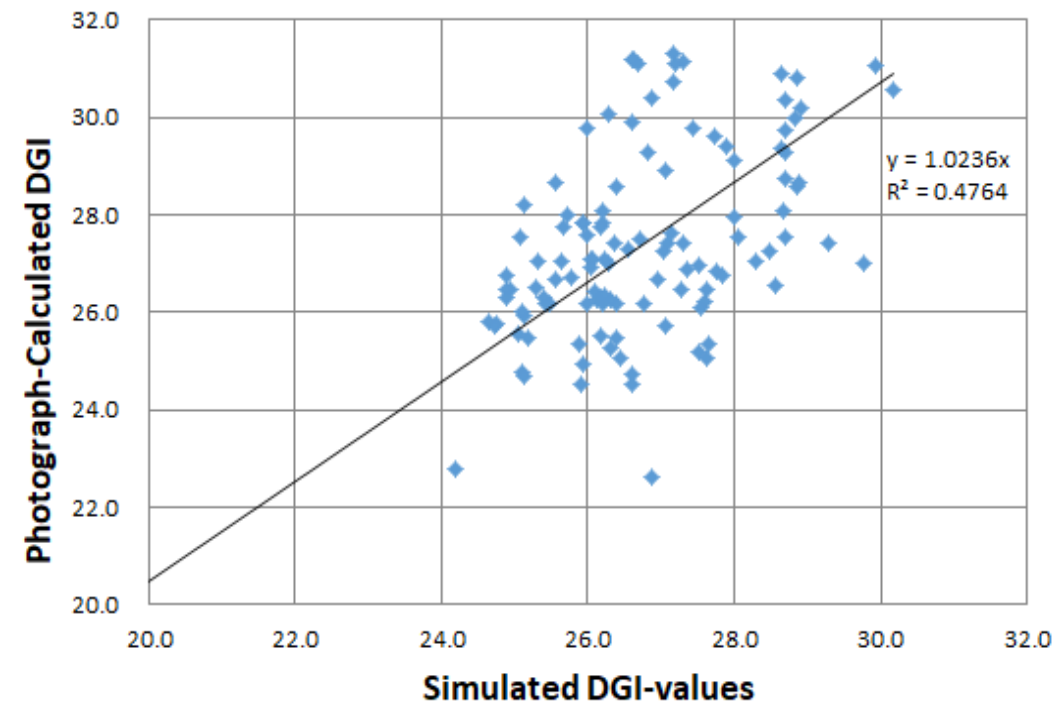

Figure 32: Comparison of simulated DGI-values with Photograph-calculated DGI-values

\section{Discussion: Pros and Cons}

The generation of HDR photographs from LDR photos and calibration is a long process and needs professional experiences. The method can be applied to all daylighting application scenarios.

Using the Radiance calculation method needs laboratory measured BSDF data and measured real-time weather data of direct normal and horizontal diffuse irradiances information. Both methods present a good agreement on DGP values and subjects vertical eye illuminance values, but the calculated DGI values with two methods shows much larger variations. 


\section{9 pab advanced technologies Ltd}

by Peter Apian-Bennewitz, pab Ltd

The PG2 gonio-photometer at pab Ltd was first built in 2004 for our own consulting work in the fields of architectural glazings and general engineering. Its concept was based on my diploma/PhD work for daylighting materials at Fraunhofer ISE going back to 1990. We continue to use our PG2 in-house as well as supporting PG2 machines which have been delivered to clients.

The PG2 is a mechanically scanning, out-of-plane, gonio-photometer covering reflection and transmission. A modular design allows adaptation to different requirements, covering a wide range of BSDF types. Materials have varied from cloth, paper, retro-reflectors, paint, to polished mirrors and lightredirecting glazing materials. Most of our work concerns visual aspects, rather than thermal or energy aspects. Therefore, a discrete BSDF representation based on the traditional Klems angles has been of very limited use to us.

It seems worth to recall some basic advantages of a scanning gonio-photometer over image-based systems. These generic aspects lead to the operating principle of the PG2.

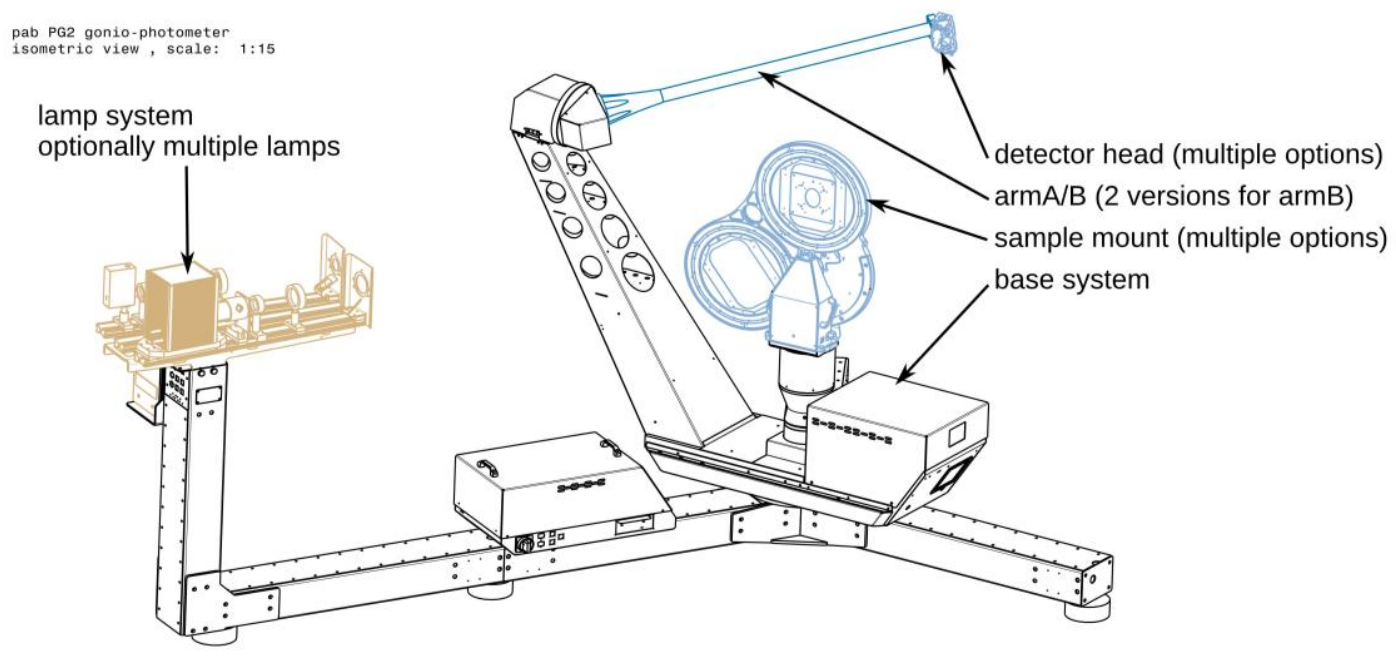

Figure 33: Components in PG2 gonio-photometer

\section{Key feature of the principle of a scanning goniophotometer}

- no intermediate components in beam path that add to scattering and crosstalk, offering a lower error budget and a detailed understanding of residual error sources

- clear separation of angular position and signal acquisition

- detector response identical at all positions

- detector can be verified externally ("in-vitro")

- optional multiple detectors read in parallel (either different signal ranges, spectral filters, different apertures, etc.)

- detectors and detector materials are adaptable to task (spectral range, solid angle)

- instrument signature is well defined and makes machine suitable as primary BSDF reference. PG2 data has been used to calibrate other BSDF methods.

\section{PG2 key features}

- measurement-on-the-fly while detector is moving offers high speed (1k samples/sec)

- measurement of unscattered beam as reference, no "diffuse" standard required 
- modular and adaptable: lamp system, sample mount, detector options available

- low self-shading by sample mount and detector arms

- adaptable optical angular resolution, down to $1 \mathrm{mrad},\left(3.4^{\prime}\right)$ using filtered laser and $1 \mathrm{~mm}$ aperture. Mechanical angular encoder resolution is 6". For further details on accuracy see PG2 datasheet.

\section{Data flow of measurement and modelling}

- unpacking sample, visual inspection to estimate scan path and incident steps. Routinely all materials are treated as "non-isotropic", until initial measured data shows a negligible dependence on phi_in

- $\quad$ PG2 measurements

- automated, script-based generation of visualizations, integrated values and 2D cuts through BSDF data check of BSDF visualizations for correct angular resolution and possible artefacts

- if client requires BSDF data only, or a comparison of BSDF of materials for her/his project, the BSDF visualizations and project-focused interpretations are compiled into a report.

- if client requires a model for a simulation tool, one example is Radiance, this is mostly supplied as a model built with mathematical standard functions, whose parameters are fitted to the measured data. The selection of a "good" functional model is largely heuristic but matches the underlying scattering principles and symmetry in a material. A practical model uses 5-10 parameters, A functional model does involve the manual selection of functions, but this approach, established in the authors' PhD 1995, still offers advantages over newer ideas (see below).

- the model is then verified a second time within the simulation program ("in-vitro") by modelling a gonio-photometer and comparing the results with the BSDF data that was initially measured. 2D cross-sections were found to be most illustrative for this. The test also ensures that the coordinate system used in the functional file is correct.

\section{Pros and cons of functional models}

\section{(i) Pros:}

- Compactness: A functional model with its parameters is an extremely compact description of a BSDF: A typical function file for radiance has size of a few kBytes and a few dozen lines of text. The author's most complex one, built for a project, had, including comments, 3kB and 108 lines.

- Robust: A functional model works for any incident and outgoing angle. It interpolates intrinsically between measured angles, and, if done carefully, extrapolates to angles that are not directly available for measurement, e.g., retro reflection towards light-source and angles in selfshadowed regions of the measurement machine. The model uses some, at least crude, insight into the scattering of a material. And a knowledge-based approach is almost always more effective than an automated ab-initio "catch-all" data-processing. This is especially true for the BSDF of daylighting- and light-redirecting systems, as they are characterized by "odd", nonstandard, shapes of the BSDF ("rings", secondary peaks, etc.).

- Simple to test, communicate and use: Data processing occurs in clearly defined steps and can be cross-checked at each step. Results are easy to document and communicate, enhancing reliability of the BSDF handling for clients. Traceability of results is highly valuable in commercial consulting.

- It is inter-operable: Most simulation tools in optics and lighting support user-defined functions for the BSDF, making it relatively simple to re-use a BSDF model in a different tool.

- It takes advantage of the PG2 design concept of asymmetric BSDF measurement: A low number of incident angles, combined with a finely resolved measurement of outgoing angles, including well-resolved peaks. This relies on the fact that features of an BSDF "move" in a predictable way with the incident angle. The method requires only a small number of incident angles, and hence PG2 measurements. 
- Adaptive angular resolution is built into the method.

- Introduces no sampling artefacts into the BSDF data.

- Eliminates noise in areas where BSDF is very low.

- Depending on the simulation tool, the approach offers the separation of a "finite" part of the BSDF (scattered light) and one (or multiple) "delta functions", which represent light that is transmitted unscattered through the material, or redirected to an arbitrary direction with negligible scattering. This can provide great advantages for an effective simulation and an accurate representation of the material. In the case of the PG2, extraction these "delta-function" parts in a BSDF is greatly helped by knowledge of the beam profile itself, since it is measured with high angular resolution as reference anyway.

\section{(ii) Cons:}

- Not scalable: Using a functional model depends crucially on "manually" envisioning a suitable model for each type of sample at hand. This does not scale well and make the method less suitable for automated batch processing of a large number of different sample types.

- Additionally, automated methods to find a set of functions for a given dataset do exist but would likely result in an agglomerate of elementary functions that is hard to decipher, requiring a more thorough verification of the final BSDF model. Yet, the concept of automated function finding is interesting to look into.

\section{Examples}

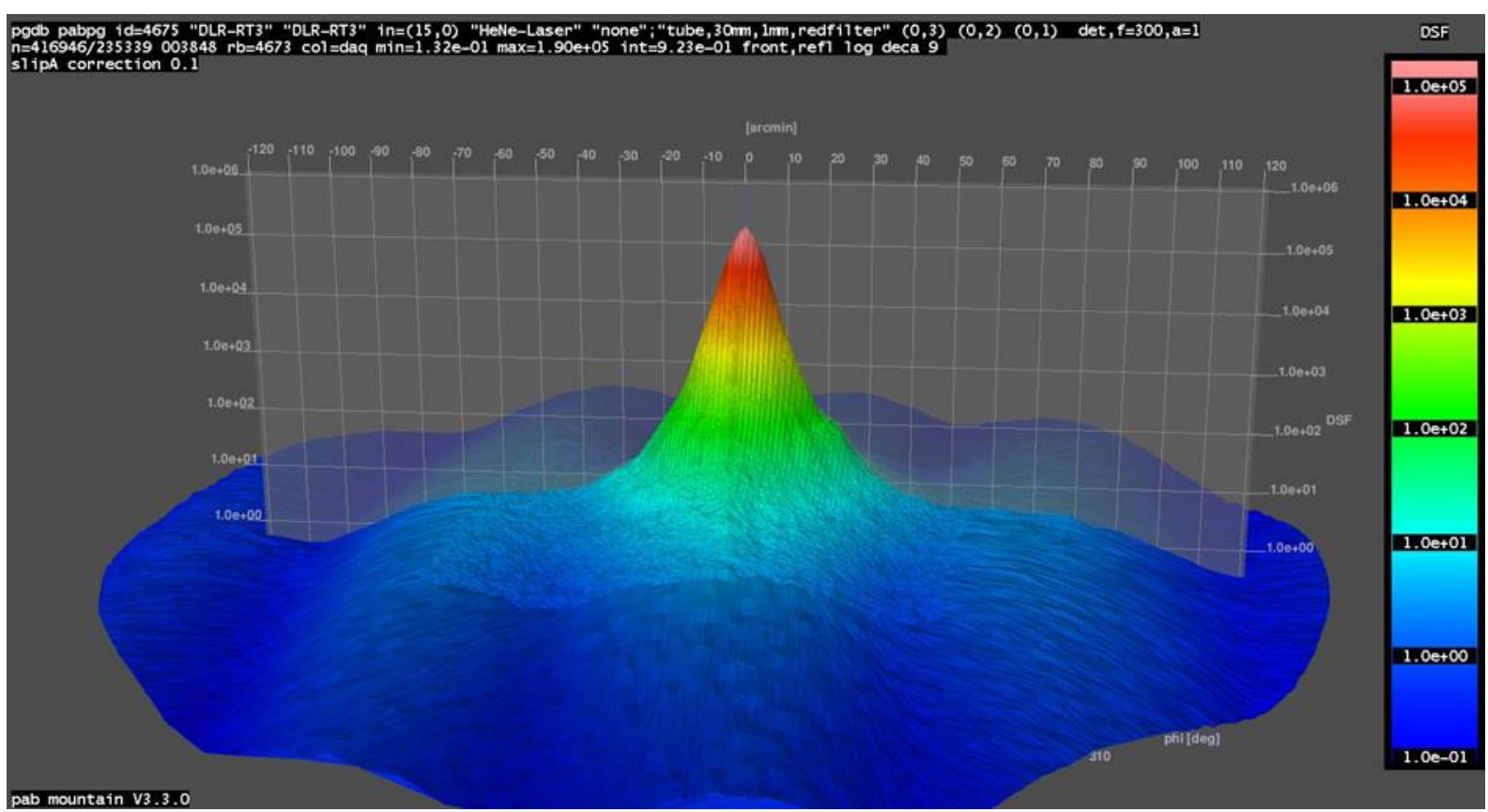

Figure 34: BSDF high resolution Mountain plot, mirror for solar concentrators. DLR project 2012. The whole plot shows an area of $2 \mathrm{deg}$ around the ideal reflected peak. The angular grid has a step size of 10 arc-minutes. 


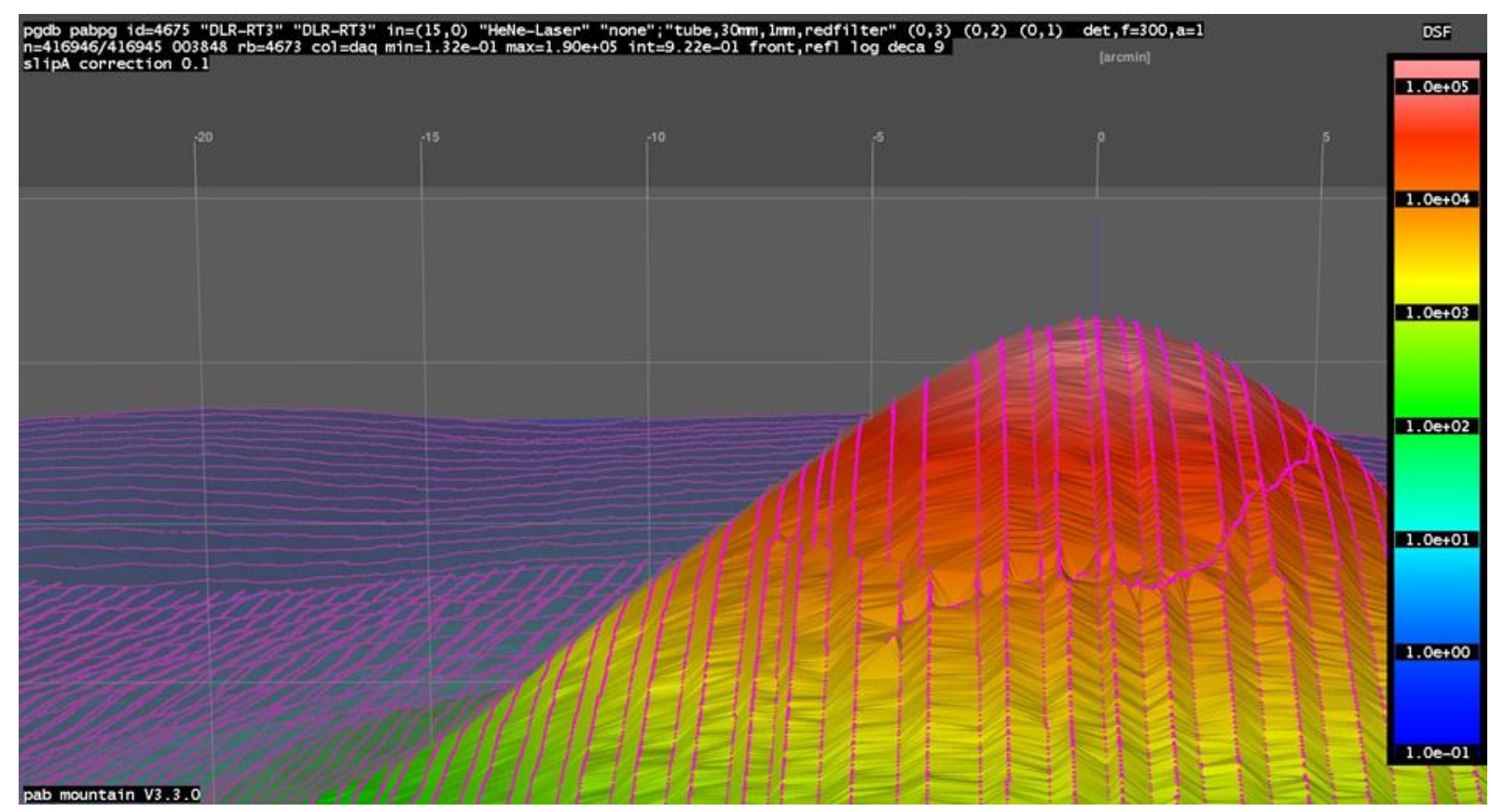

Figure 35: BSDF high resolution: Close-up of mirror dataset, angular step size 5 arc-minutes. Data-points are shown as little dots, spaced 4" along scan lines and 40 " between scan lines. Measurement used a spatially filtered HeNe laser beam focused on a $1 \mathrm{~mm}$ aperture at the detector

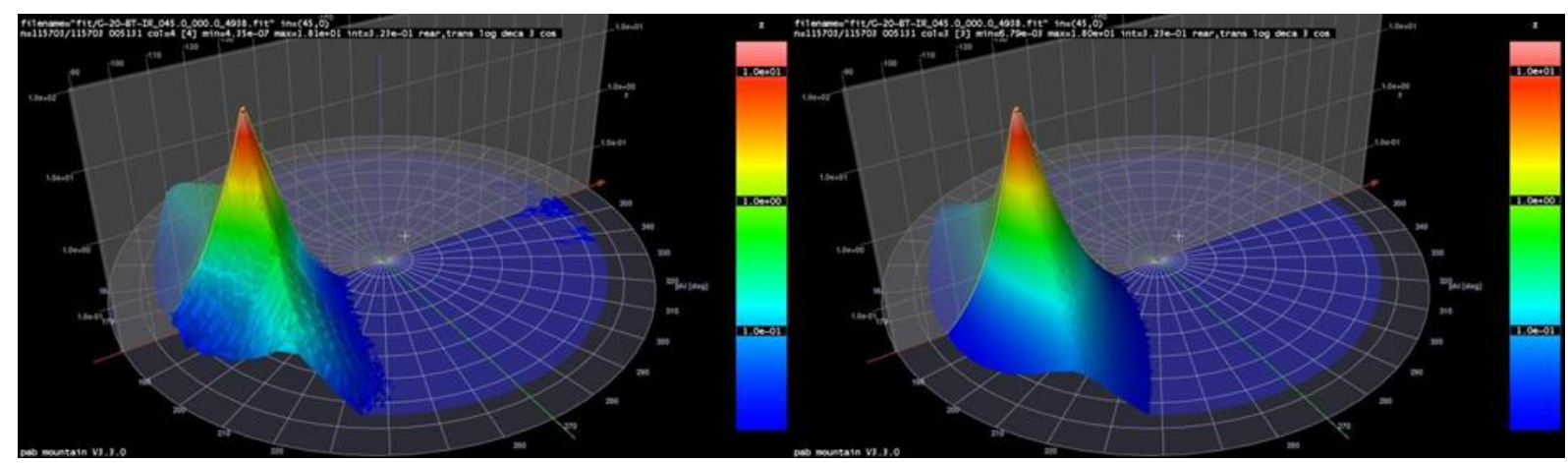

Figure 36: BSDF Mountain plot of the transmitted hemisphere, data of a translucent, structured roofing material, incident angle (45deg, 45deg). Plot on left shows measured data, plot on right shows data of a custom BSDF model with its parameters fitted to the measured data. 


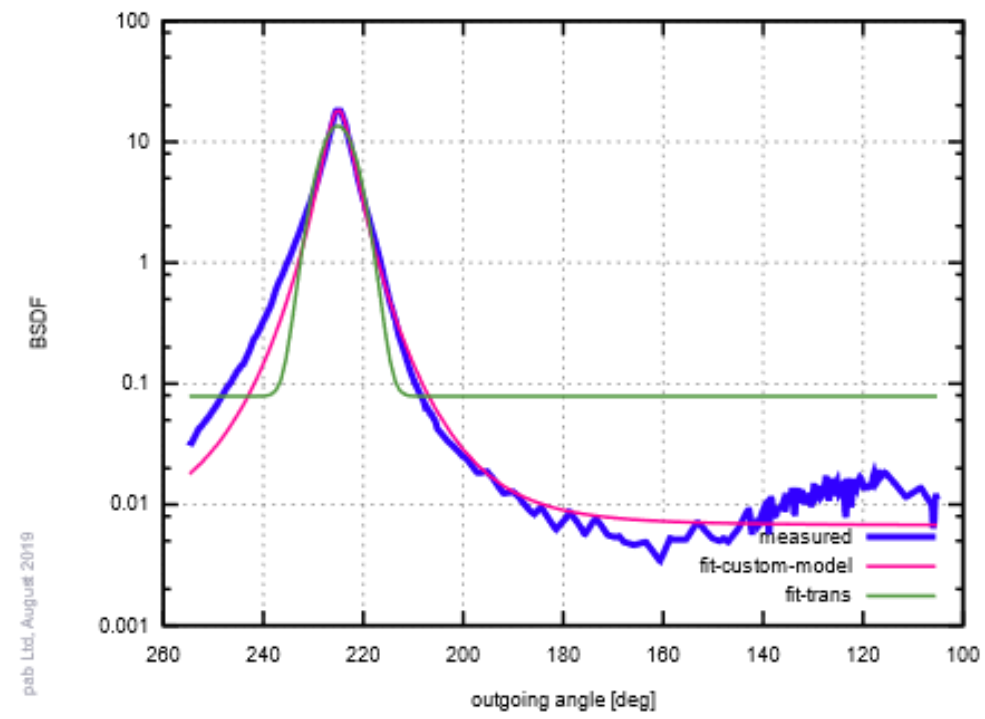

Figure 37: 2D cut along the plane-of-scattering through above example data set, comparing measured data and two models. The built-in model (dashed line) mismatches the peak shape and BSDF values below $1 \%$ of the maximum, while the custom model approximates both better.

References are the author's website ${ }^{42}$ and pab documentation ${ }^{43}$.

${ }^{42}$ pab advanced technologies Ltd, website, http://www.pab.eu/

$43 \mathrm{pab}$ - Documentation, articles, papers, online http://www.pab.eu/docs/?C=M;O=D 


\subsection{Comparison of BSDF generation methods}

\subsubsection{Measurement devices}

\begin{tabular}{|l|l|l|l|}
\hline \multicolumn{2}{|l|}{ Scanning goniophotometer } \\
\hline $\begin{array}{l}\text { pab goniometer PG2 } \\
\text { www.pab.eu/gonio- } \\
\text { photometer/ }\end{array}$ & Key features & $\begin{array}{l}\text { Data format provided } \\
\text { by device software }\end{array}$ & Laboratories \\
\hline & $\begin{array}{l}\text { versatile BSDF } \\
\text { equipment with high } \\
\text { signal range and } \\
\text { high angular } \\
\text { resolution }\end{array}$ & Tabular ASCII & $\begin{array}{l}\text { Fraunhofer ISE } \\
\text { Freiburg } \\
\text { HSLU Lucerne } \\
\text { LBNL Berkeley } \\
\text { Pab } \\
\text { Realistic Graphics Lab } \\
\text { at EPFL Lausanne }\end{array}$ \\
\hline
\end{tabular}

Description:

- Manufactured by pab advanced technologies Ltd.

- Mechanical scanning of a detector head with multiple sensors over a virtual sphere surface that is centred on the intersection between the incident beam and the sample.

- The sample can be rotated around vertical and horizontal axes to allow a broad range of incidence angles.

- Diameter of virtual sphere scanned by detector: $1 \mathrm{~m}$

- $\quad$ Light sources: halogen and xenon lamps, collimated LEDs

- Detector options: silicon diode, optionally filtered, InGaAs (IR up to 2.5um), SiC (UV)

- Minimum BSDF: $<10 \mathrm{e}-4$

- Dynamic range: $10 \mathrm{e} 9$ electronically, with same beam profile, more if multiple beam profiles are combined (e.g. laser + Xenon light sources)

- Modular design, multiple lamp systems and sample mounts are available

- Unscattered beam measurement as reference

- Adaptive BSDF scanning

Further information:

- $\quad$ http://www.pab.eu/docs/PG2-flyer-en.pdf

- http://www.pab.eu/docs/PG2-advantages.pdf

\begin{tabular}{|l|l|l|l|}
\hline $\begin{array}{l}\text { Reflet } 180 \\
\text { www.lighttec.fr/scattering- } \\
\text { measurements/ }\end{array}$ & Key features & $\begin{array}{l}\text { Data format provided } \\
\text { by device software }\end{array}$ & Laboratories \\
\hline & $\begin{array}{l}\text { Compact equipment } \\
\text { with high dynamic } \\
\text { range and high } \\
\text { precision }\end{array}$ & $\begin{array}{l}\text { Text files } \\
\text { LightTools (opr) }\end{array}$ & LightTec \\
& & \\
\hline
\end{tabular}




\begin{tabular}{|c|c|c|c|}
\hline \multicolumn{4}{|c|}{ Image based goniophotometer } \\
\hline $\begin{array}{l}\text { IBP / EPFL } \\
\text { www.ibp.fraunhofer.de } \\
\text { www.epfl.ch }\end{array}$ & Key features & $\begin{array}{l}\text { Data format provided } \\
\text { by device software }\end{array}$ & Laboratories \\
\hline & $\begin{array}{l}\text { Comprehensive } \\
\text { BTDF monitoring }\end{array}$ & IEA 21, Text files & $\begin{array}{l}\text { EPFL Lausanne } \\
\text { Fraunhofer IBP } \\
\text { Stuttgart }\end{array}$ \\
\hline \multicolumn{4}{|c|}{ 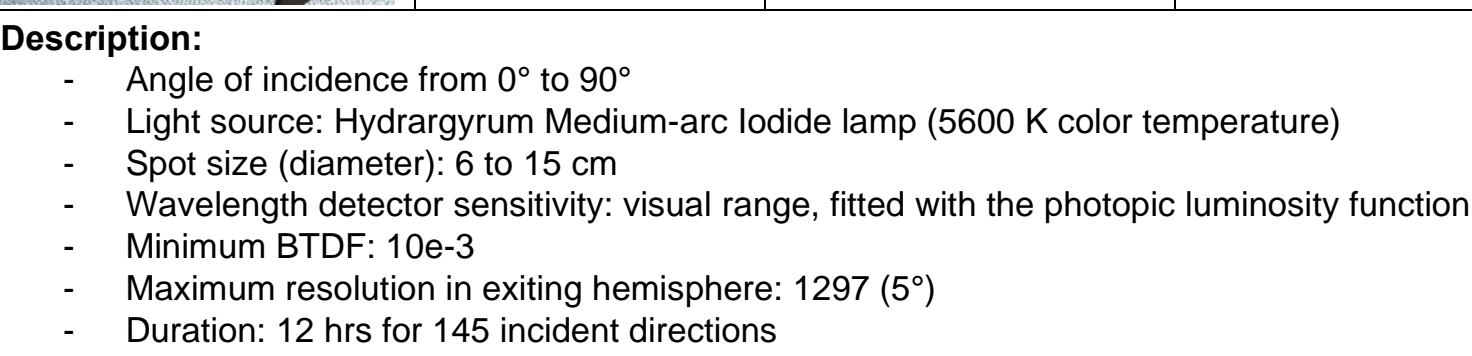 } \\
\hline $\begin{array}{l}\text { Mini Diff v2 } \\
\text { www.lighttec.fr }\end{array}$ & Key features & $\begin{array}{l}\text { Data format provided } \\
\text { by device software }\end{array}$ & Laboratories \\
\hline & $\begin{array}{l}\text { Portable equipment, } \\
\text { easy to use and fast }\end{array}$ & $\begin{array}{l}\text { Text files (ASTM, mesh, } \\
\text { slice), Light Tools, } \\
\text { Radiant Zemax, Speos, } \\
\text { Trace Pro; ABg (Harvey } \\
\text { Shack) and Gaussian } \\
\text { fits }\end{array}$ & $\begin{array}{l}\text { LightTec } \\
\text { Bartenbach }\end{array}$ \\
\hline \multicolumn{4}{|c|}{$\begin{array}{cl}\text { Description } & 44: \\
\text { - } & \text { Angle of incidence: fixed at } 0^{\circ}, 20^{\circ}, 40^{\circ}, 60^{\circ} \text { (BRDF and BTDF) } \\
\text { - } & \text { Angular aperture: }-75^{\circ} \text { to }+75^{\circ} \text { (hemispherical measurement) } \\
\text { - } & \text { Light source: } 3 \text { color collimated sources (RGB) at } 465 \mathrm{~nm}, 525 \mathrm{~nm} \text { and } 630 \mathrm{~nm} \\
- & \text { Spot Size (diameter): } 1 \mathrm{~mm} \\
- & \text { Wavelength detector sensitivity: } 3 \text { channels red, green and blue (RGB) } \\
\text { - } & \text { Dynamic range: } 10 \mathrm{e} 5 \\
- & \text { BSDF Accuracy }<5 \% \text { (for Lambertian sample) } \\
- & \text { BSDF Repeatability }<2 \% \text { (for Lambertian sample) } \\
- & \text { Color Accuracy Duv }<0.1 \\
- & \text { Angular Aperture }-75^{\circ} \text { to }+75^{\circ}\end{array}$} \\
\hline
\end{tabular}

44 LIGHT TEC: MINI-DIFF V2, For 2D/3D scattered light measurements, Preliminary datasheet. http://www.lighttec.fr/wp-content/uploads/2017/10/Flyer-Mini-Diff-V2.pdf (accessed 24 January 2019). 


\begin{tabular}{|l|l|l|l|}
\hline \multicolumn{3}{|l|}{ Integrating sphere } & Key features \\
\hline $\begin{array}{l}\text { Integrating sphere } \\
\text { (Ulbricht sphere) }\end{array}$ & $\begin{array}{l}\text { Data format } \\
\text { provided by } \\
\text { device software }\end{array}$ & Laboratories \\
\hline & $\begin{array}{l}\text { Measurement of integrated } \\
\text { flux, i.e., spatially integrated } \\
\text { transmittance or reflectance } \\
\text { for daylight systems. } \\
\text { Available for spectrally } \\
\text { resolved and spectrally } \\
\text { integrated (broadband) } \\
\text { measurements. }\end{array}$ & $\begin{array}{l}\text { Varies } \\
\text { (depending on } \\
\text { manufacturer) }\end{array}$ & $\begin{array}{l}\text { Fraunhofer IBP } \\
\text { LBNL Berkeley } \\
\text { HSLU Lucerne }\end{array}$ \\
\hline
\end{tabular}

\section{Notes on the topic of accuracy and precision in measurements}

The various measuring devices discussed differ significantly from each other. This requires a critical examination of relevant influencing factors affecting accuracy and precision of the measurements. Some general information on accuracy and precision is explained understandably for example here ${ }^{46}$.

The following list is intended to encourage people not to ignore this issue but does not claim to be exhaustive. Among others, important points include:

- mechanical angular resolution of scanning goniophotometers

- resolution given by pixel density of image-based goniophotometers

- reproducible positioning of scanning goniophotometers

- angular precision of imaging system

- opening angles of detector and light source

- cross-talk between directions in image-based devices (e.g., by scattering in the lens system and/or cross-talk at chip level)

- $\quad .$.

To make instruments and measurements more comparable, one approach would be the concept of a so-called "instrument signature". Here the BSDF of an ideal sample (i.e., without a sample for transmission, and with a surface mirror sample of negligible scattering for reflection) would result in something that allows a relatively easy comparison between instruments and subsequently between taken measurements. This concept should be reviewed and elaborated in future work.

\footnotetext{
45 Multifunktionale Ulbricht-Kugel, Fraunhofer-Institut für Bauphysik IBP, 2018, online: https://www.ibp.fraunhofer.de/content/dam/ibp/ibp-neu/de/dokumente/ibpmitteilungen/551-600/557.pdf 46 See also: https://en.wikipedia.org/wiki/Accuracy and precision
} 


\subsubsection{Simulation software}

\section{BSDF generation software}

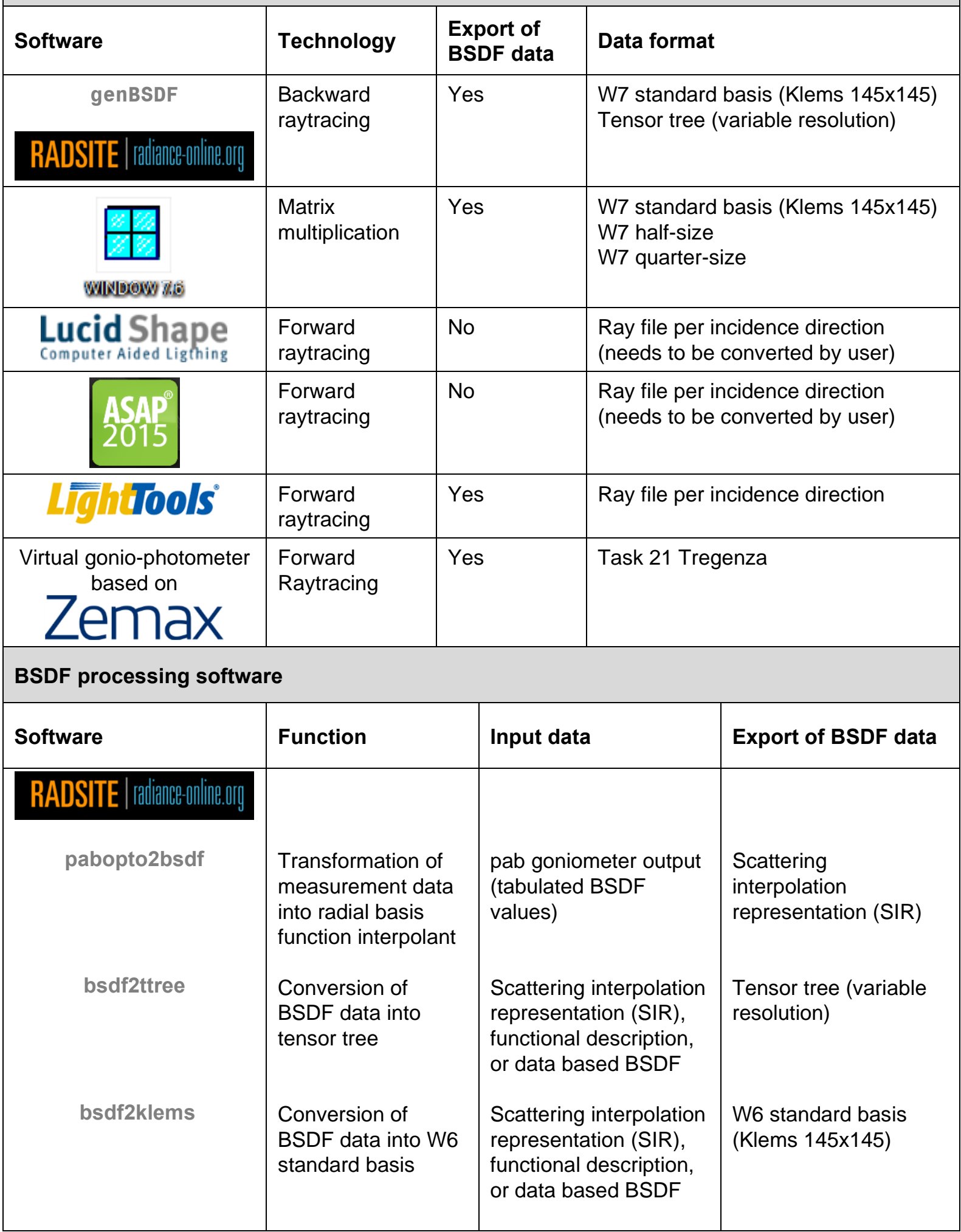




\section{BSDF Round Robin Test}

The Task experts contributing to work item C2 agreed to perform a round robin test. The objective was to assess the comparability of BSDF data sets generated by various laboratories as well as - for practical use in daylighting design even more important - the comparability of daylight performance metric evaluations based on these data.

Representing widely used shading and glare protection systems, two daylighting systems were selected: one outdoor venetian blind system and one interior textile roller blind. The contributing partners were asked to generate BSDF data sets for samples of these systems according to their usual routine. The data sets were collected and analyzed and - after partly multiple feedback rounds - applied in daylighting simulations to evaluate point-in-time and annual daylight performance metrics.

\subsection{Daylighting systems: Test samples}

Samples for the two selected daylighting systems were provided for the round robin comparison. As the BSDF data set usually describes "center-of-system" properties (comparable to thermal center-of-glass properties) of the daylighting system and any frame is modeled in the final window geometry as part of the building's CAD model, the samples were made to cover a representative part of the systems.

\subsubsection{HELLA Outdoor blind ARB 80}

The first daylighting system was the HELLA ARB 80 outdoor blind system (Figure 38, left). With a width of $80 \mathrm{~mm}$ the system can be seen as a "macroscopic system" (for a detailed discussion of classes of systems and a categorization refer to Task 61's BSDF white paper ${ }^{47}$ ). For such systems most laboratories follow a hybrid approach with measurements for the base material and simulations of the system to generate the BSDF data sets. IBP Stuttgart measured the overall macroscopic system using a special sample holder. Therefore, HELLA provided samples of the shaped slats (Figure 38, center), samples of the flat base material (Figure 38, right), and a CAD drawing specifying the system geometry (Figure 39).
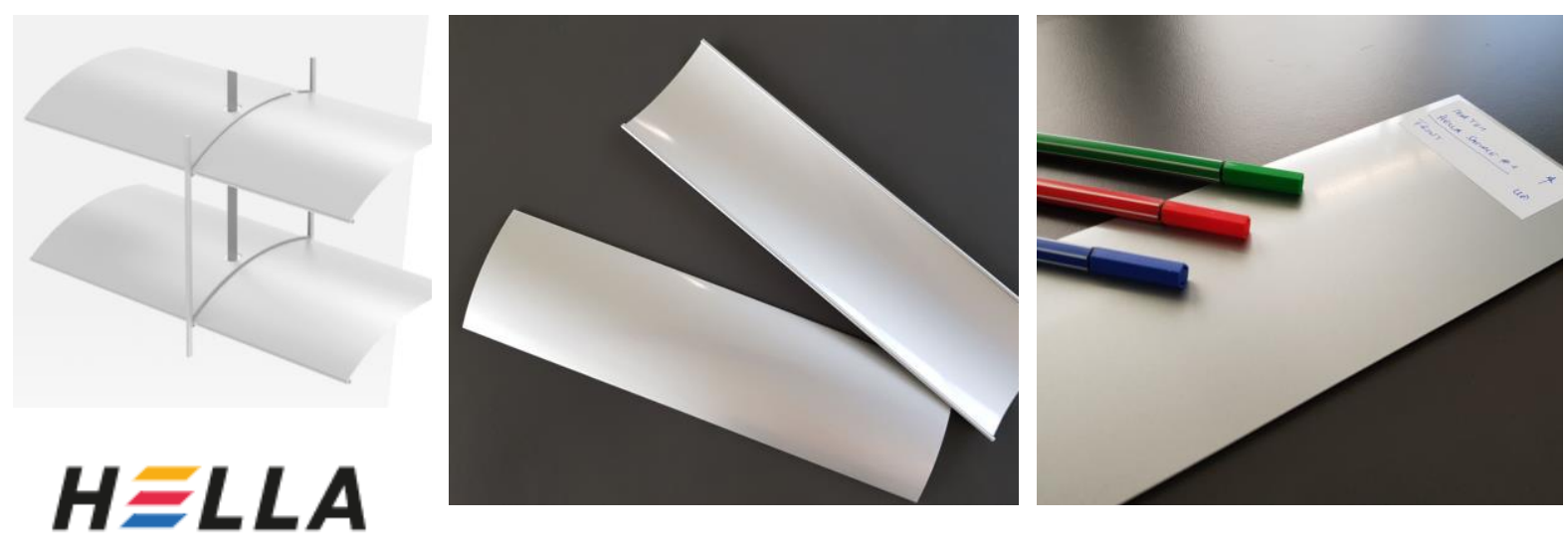

Figure 38: Round robin test system HELLA outdoor venetian blind ARB80.

Product image (left; @ HELLA Sonnen- und Wetterschutztechnik GmbH), test samples of shaped slats (center), sample of base material (right).

47 D. Geisler-Moroder, E.S. Lee, G. Ward, B. Bueno, L.O. Grobe, T. Wang, B. Deroisy, H.R. Wilson. BSDF generation procedures for daylighting systems. White paper. T61.C.2.1 - A Technical Report of Subtask C, IEA SHC Task 61 / EBC Annex 77. https://task61.iea-shc.org/publications. 2021. 


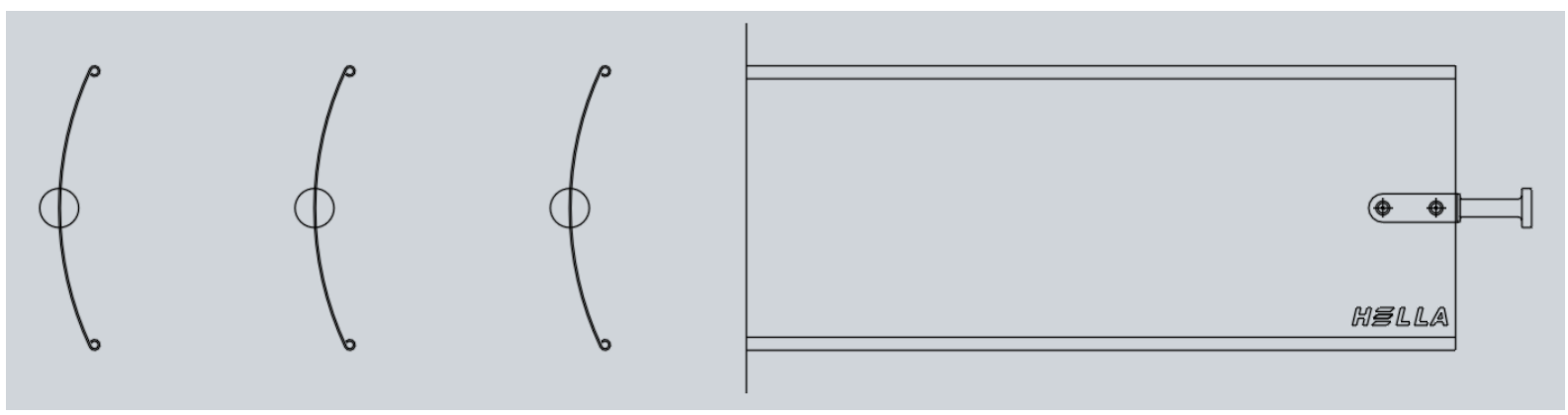

Figure 39: CAD geometry model of round robin test system HELLA outdoor venetian blind ARB80, (c) HELLA Sonnen- und Wetterschutztechnik GmbH.

As the main interest was in the effect on daylight performance metric calculations, the main request was for the BSDFs of the final system, i.e., the shaped and arranged slats, not the base material. To minimize confusion in the arrangement, we further assumed a horizontal setting of the slats, i.e., a tilt angle of $0^{\circ}$.

\subsubsection{MechoShade EuroTwill 1\% Open Slate (6216)}

The second system for the round robin test was the solar shade fabric MechoShade EuroTwill 6216 Slate with $1 \%$ openness factor (Figure 40 , left). With a thickness and structure size in the millimeter range, the system can be described as a "microscopic system" (cf. ${ }^{47}$ ). Such systems can be measured directly with goniophotometers. MechoShade provided a roll of the cloth, from which A4 size samples were cut (Figure 40, right) and distributed among the partners.
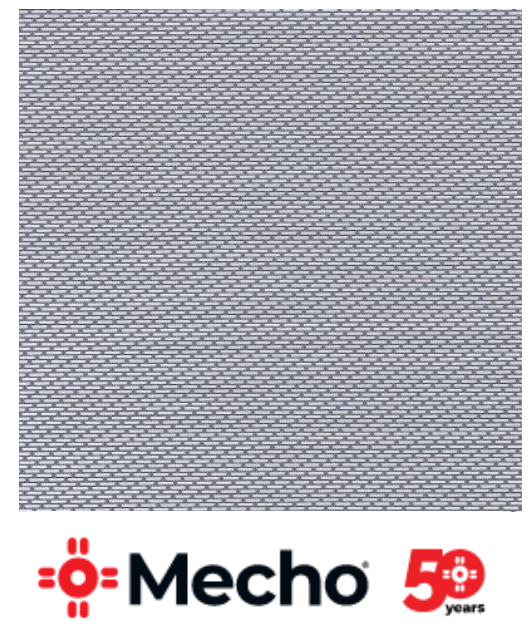

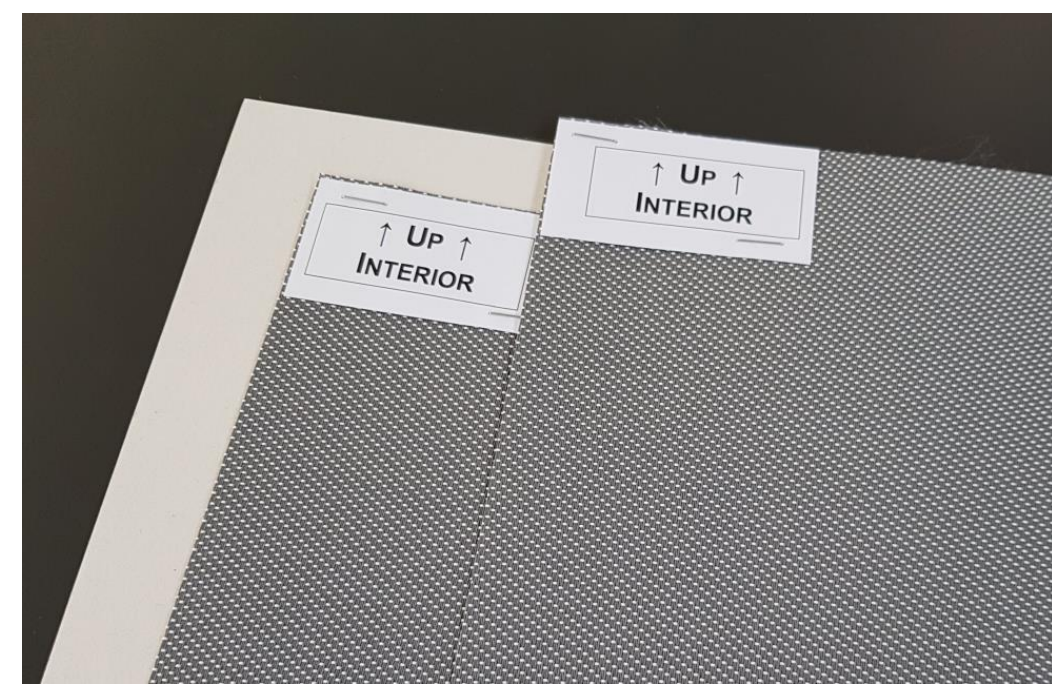

Figure 40: Round robin test system MechoShade interior textile roller blind.

Product image of exterior surface (left; @ MechoShade Systems, LLC.), interior surfaces of test samples (right).

As can be determined by visual inspection (Figure 40, left), this sample does not feature symmetry by reflection in any plane but does feature rotational symmetry of order 2 . 


\subsection{Contributing laboratories and provided data sets}

All Subtask C2 partners were invited to contribute to the round robin comparison. To ensure good comparability, all parties were asked to provide the following BSDF related data sets for use in daylighting simulations:

- $\quad B S D F$ in Klems resolution; minimum requirement BTDF, and

- BSDF high resolution data sets in the anisotropic tensor tree format with a maximum resolution of 4096x4096; minimum requirement BTDF.

Additionally, for informational purposes, participants were asked to provide

- raw measurement data and

- BSDF data sets as would typically be delivered in response to an order for BSDF generation by a daylighting system manufacturer

to document current common approaches and analyze the compatibility of BSDF processing software with various raw data formats.

The final decision about the test samples was made during the Task meeting in March 2020. Due to the Covid-19 crisis and the resulting lockdowns, the shipment and distribution of the test samples took until beginning of summer 2020. Following the restrictions, closed labs, and induced resource bottlenecks, BBRI and Hunan University unfortunately had to cancel their participation in the round robin test. Thus, no data sets from these institutes are included in the comparisons.

For the first system, the HELLA ARB 80 outdoor blind, the following data sets were provided by the participants:

\begin{tabular}{|c|c|c|c|}
\hline HELLA ARB 80 & Klems & $\begin{array}{c}\text { High resolution } \\
\text { BSDF }\end{array}$ & \begin{tabular}{c} 
Other \\
\hline $\begin{array}{c}\text { Lucerne University of Applied } \\
\text { Sciences and Arts (HSLU) }\end{array}$
\end{tabular} \\
$\begin{array}{c}\text { Fraunhofer-Institut für } \\
\text { Bauphysik (FH-IBP) }\end{array}$ & $\mathrm{X}$ & $\mathrm{X}$ & $\begin{array}{c}\text { Base material raw } \\
\text { measurement data }\end{array}$ \\
\hline $\begin{array}{c}\text { Fraunhofer Institute for Solar } \\
\text { Energy Systems (FH-ISE) }\end{array}$ & $\mathrm{X}$ & $\mathrm{X}$ & $\begin{array}{c}\text { Raw measurement } \\
\text { data }\end{array}$ \\
\hline $\begin{array}{c}\text { Lawrence Berkeley National } \\
\text { Laboratory (LBNL) }\end{array}$ & $\mathrm{X}$ & $\mathrm{X}$ & $\begin{array}{c}\text { Base material raw } \\
\text { measurement data }\end{array}$ \\
\hline $\begin{array}{c}\text { EPFL - LESO-PB (EPFL) } \\
\text { Bartenbach GmbH (BB) }\end{array}$ & $\mathrm{X}$ & $\mathrm{X}$ & $\begin{array}{c}\text { IEA Task 21 format } \\
\text { (Tregenza/5deg) }\end{array}$ \\
\hline pab advanced technologies Ltd \\
(PAB)
\end{tabular}

48 Variable resolution tensor tree BSDF, max. resolution: 4096x4096. 
For the second round-robin test system, the solar shade fabric MechoShade EuroTwill 6216 Slate, the following data sets were provided by the participants:

\begin{tabular}{|c|c|c|c|}
\hline MechoShade 6216 & Klems & $\begin{array}{l}\text { High resolution }{ }^{48} \\
\text { BSDF }\end{array}$ & Other \\
\hline $\begin{array}{l}\text { Lucerne University of Applied } \\
\text { Sciences and Arts (HSLU) }\end{array}$ & $x$ & $x$ & $\begin{array}{c}\text { Raw measurement } \\
\text { data }\end{array}$ \\
\hline $\begin{array}{l}\text { Fraunhofer-Institut für } \\
\text { Bauphysik (FH-IBP) }\end{array}$ & $\mathrm{x}$ & & $\begin{array}{c}\text { Raw measurement } \\
\text { data }\end{array}$ \\
\hline $\begin{array}{l}\text { Fraunhofer Institute for Solar } \\
\text { Energy Systems (FH-ISE) }\end{array}$ & $x$ & $x$ & \\
\hline $\begin{array}{l}\text { Lawrence Berkeley National } \\
\text { Laboratory (LBNL) }\end{array}$ & $x$ & $\mathrm{x}$ & $\begin{array}{c}\text { Raw measurement } \\
\text { data }\end{array}$ \\
\hline \multicolumn{4}{|l|}{ EPFL - LESO-PB (EPFL) } \\
\hline \multicolumn{4}{|l|}{ Bartenbach GmbH (BB) } \\
\hline $\begin{array}{l}\text { pab advanced technologies Ltd } \\
\text { (PAB) }\end{array}$ & & & $\begin{array}{c}\text { Raw measurement } \\
\text { data }\end{array}$ \\
\hline
\end{tabular}

Besides the already mentioned Covid-19-related cancellations, not all laboratories provided all data sets for various reasons. Some of the reasons are:

- FH-IBP usually provides BSDF data sets in the Tregenza/5 $5^{\circ}$ IEA21 format (cf. ${ }^{47}$ ) to its clients. They took quite some effort to measure the data sets based on the Klems resolution and did not go further generating higher resolution data.

- EPFL had technical issues with its CCD based goniophotometer. Some first measurements showed that there were some issues which still need to be fixed. No final data for the fabric could be provided.

- BB can fully characterize macroscopic systems or regular scattering microscopic systems with structure sizes clearly below $1 \mathrm{~mm}$. Thus, the selected fabric lies exactly in the uncertain range in between and therefore no data was provided. BB is currently working on a measurement device to also capture samples with similar structure sizes.

- $\quad$ PAB provided an extensive set of raw data of material measurements both of the blind base material and the fabric but provided no data sets processed in common data formats for further use in simulations. PAB also provided a comprehensive analysis of the measurement procedure and setup. More information can be found on PAB's webpage ${ }^{42}$. 


\subsection{Description of analysis}

The provided BSDF data sets were analyzed and compared. The objective was to assess whether data sets from various laboratories for the same daylighting system differ and if calculations based on these data are affected by these differences. The comparison thus included an analysis of the data sets per se, focusing on the transmittance values as the crucial component for daylighting simulations, as well as the comparability of daylight performance metrics evaluations based on these data sets. In detail, the following analysis and simulations were performed:

- Comparison of transmittance data derived from the BSDF data sets,

- point-in-time simulations using the various BSDF data sets and

- annual simulations using the various BSDF data sets.

The results are also structured according to this scheme and are presented for both test samples, the HELLA blind system and the MechoShade cloth, and for all provided data sets.

To allow a direct comparison of the data sets, influences such as e.g., controls or user interaction were not considered. The exact same simulation workflow was applied to each laboratory's BSDF data sets. While this does not represent real-world situations, it enabled a focused analysis of the differences between the data sets. 


\subsection{Comparison of transmittance data from BSDFs}

\subsubsection{HELLA ARB 80}

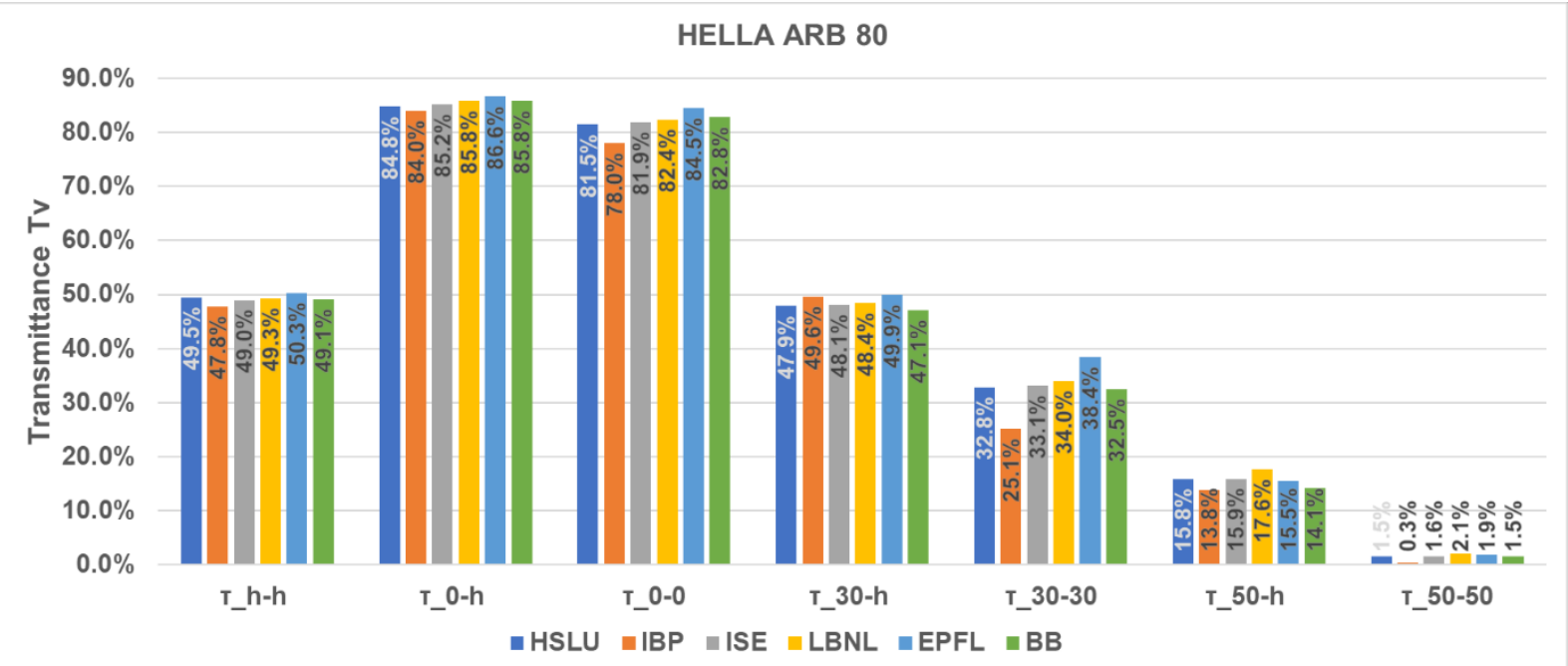

Figure 41: Evaluation from HELLA ARB $80 \mathrm{Klems}$ BSDF data sets: hemispherical-hemispherical transmittance $T_{h-h}$ as well as direct-hemispherical $T_{x-h}$ and direct-direct $T_{x-x}$ transmittance for selected angles of incidence.

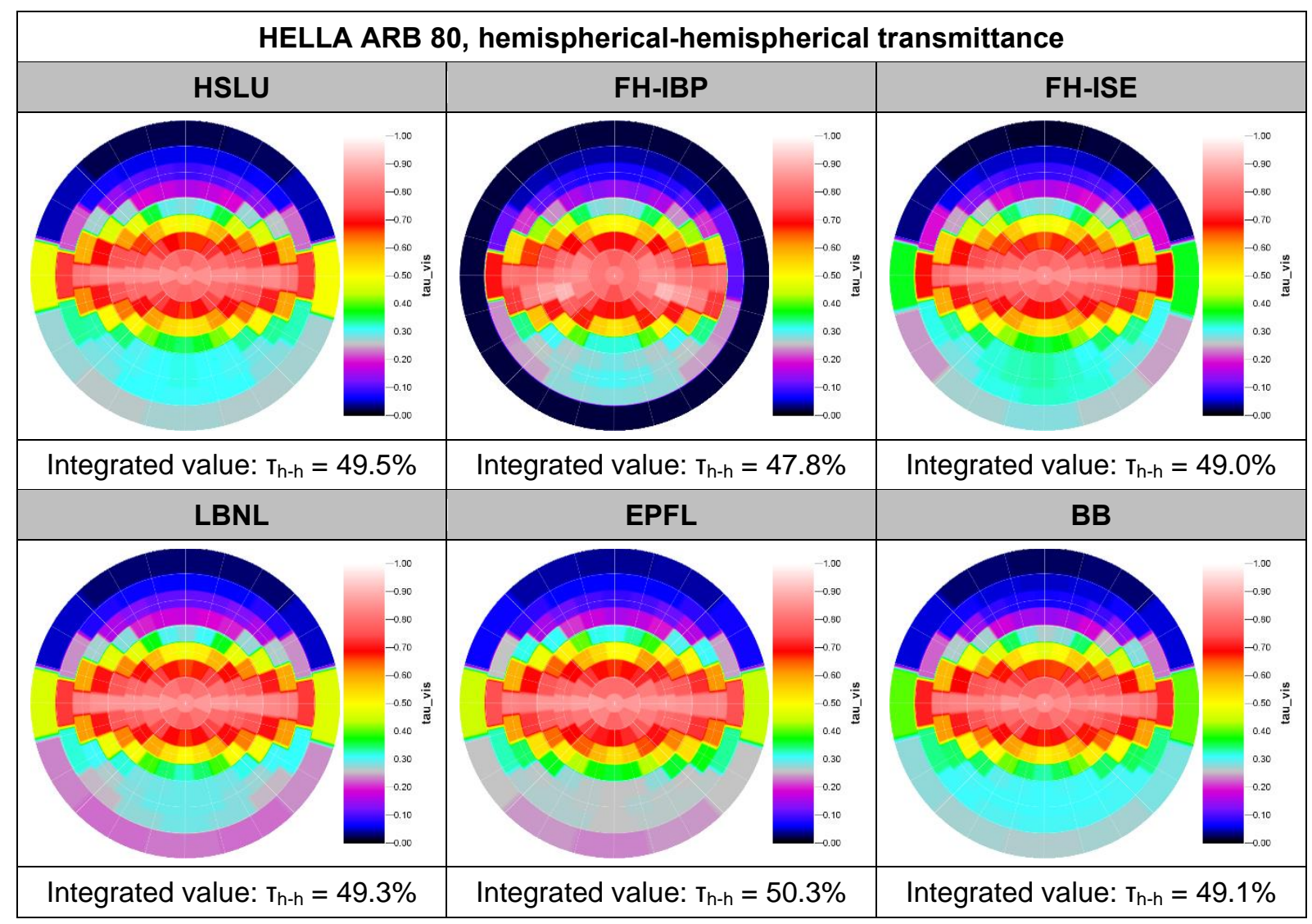



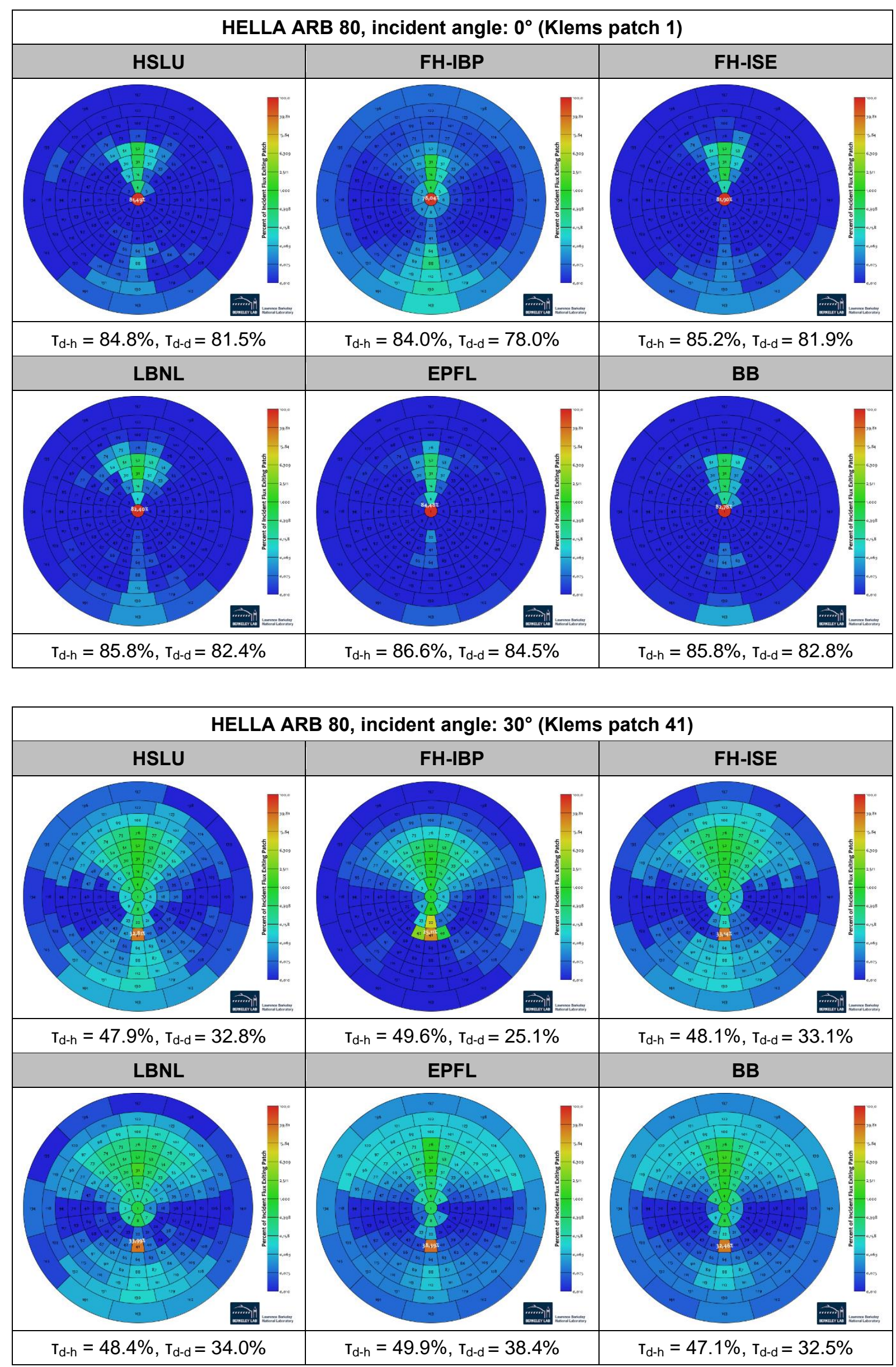


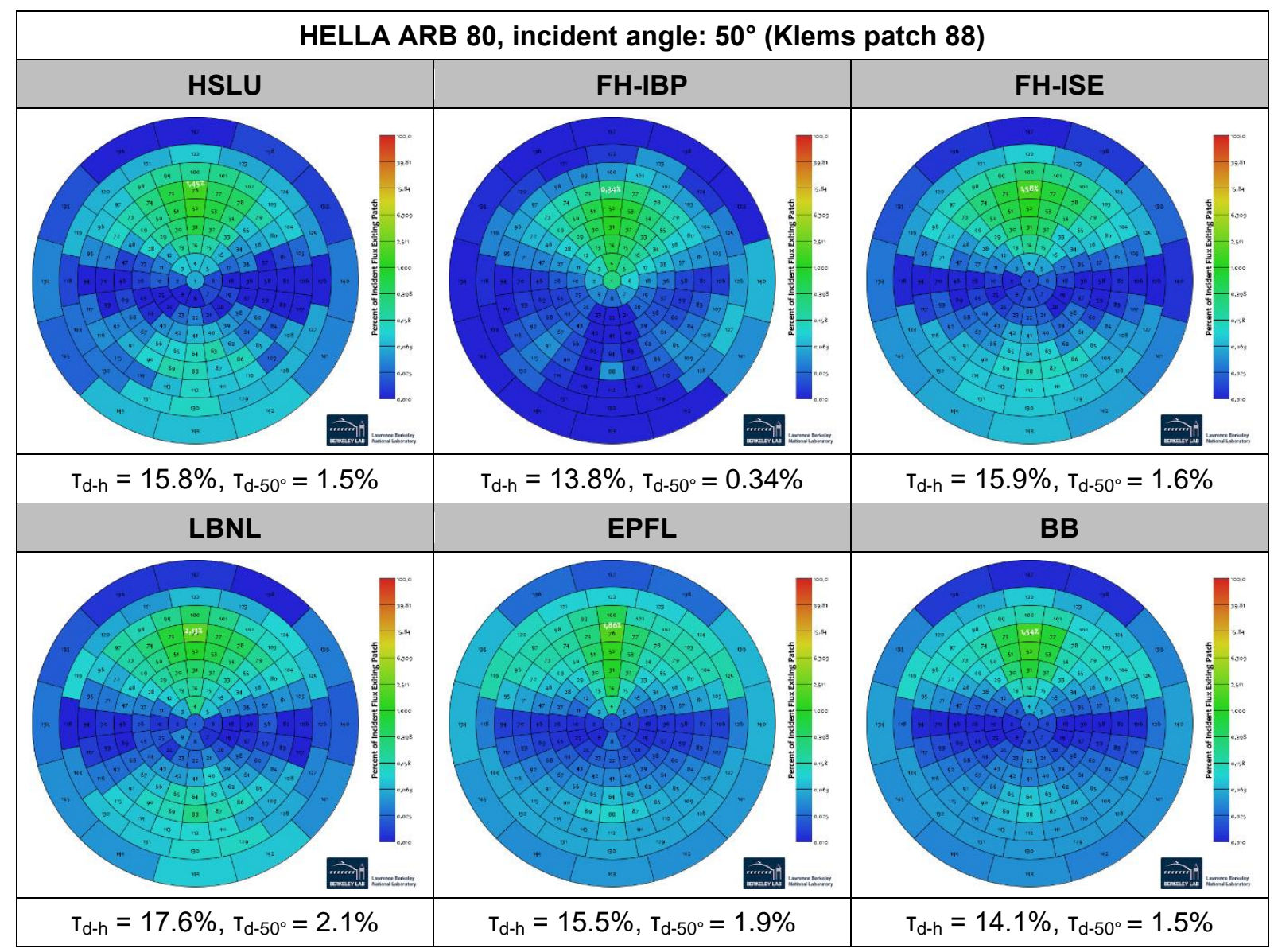




\subsubsection{MechoShade 6216}

MechoShade 6216

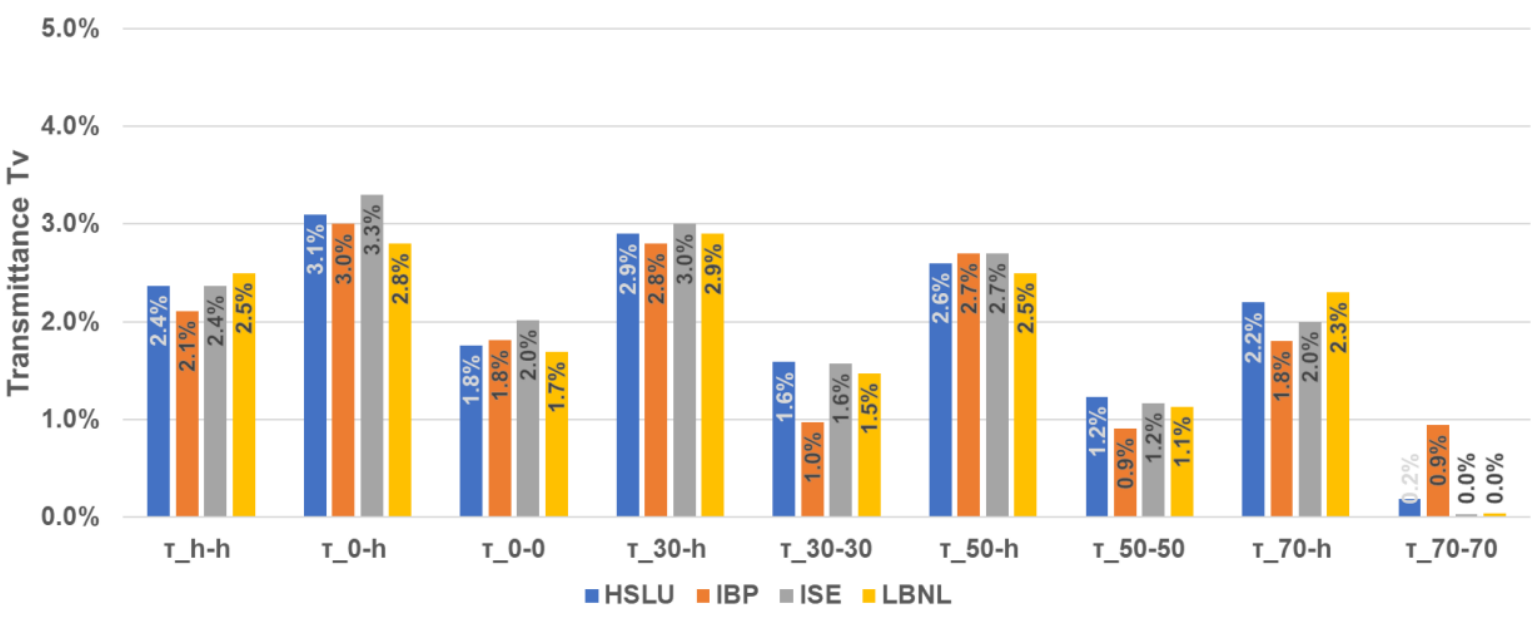

Figure 42: Evaluation from MechoShade $6216 \mathrm{Klems} \mathrm{BSDF}$ data sets: hemispherical-hemispherical transmittance $\mathrm{T}_{\mathrm{h}-\mathrm{h}}$ as well as direct-hemispherical $T_{x-h}$ and direct-direct $T_{x-x}$ transmittance and for selected angles of incidence.

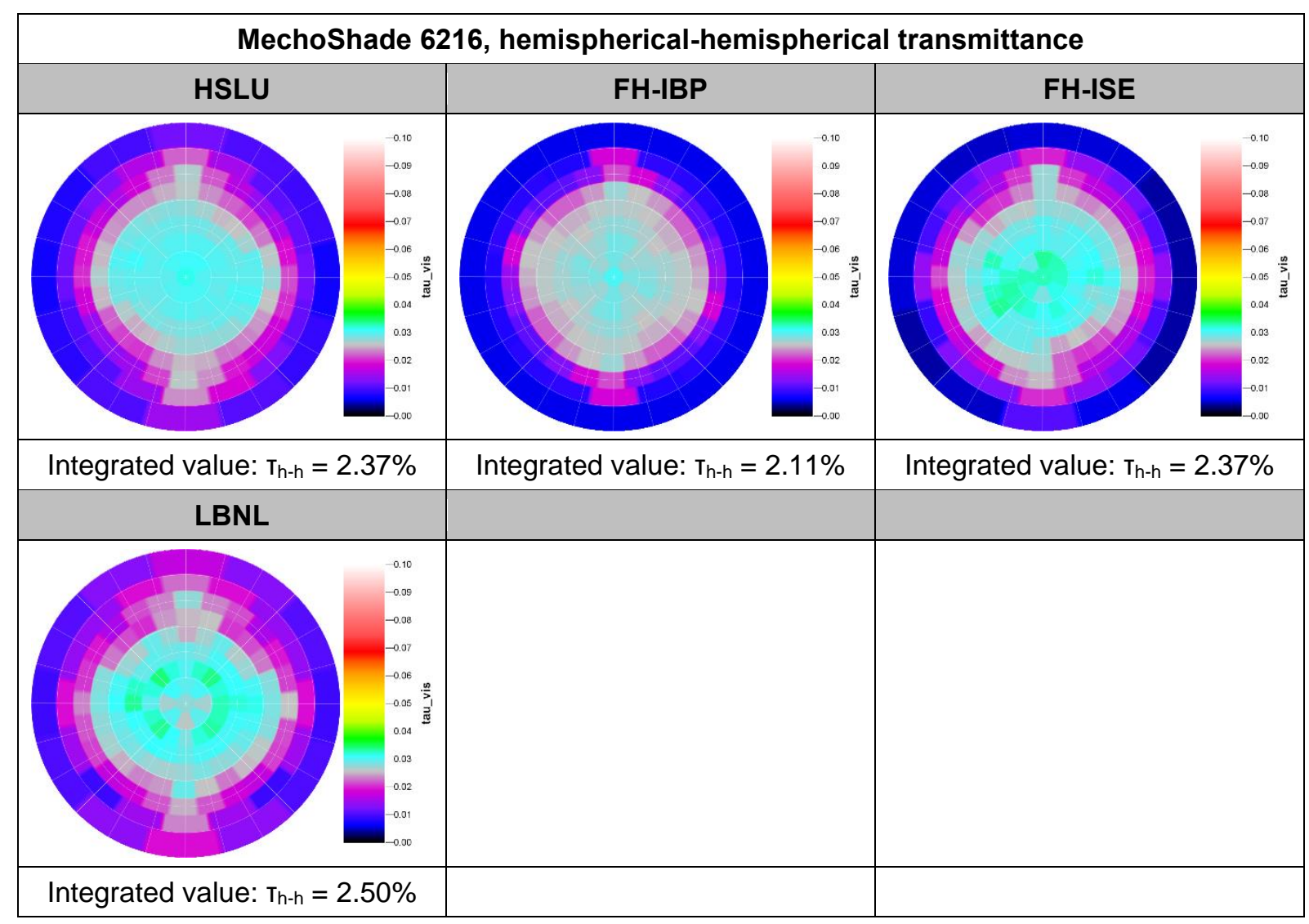



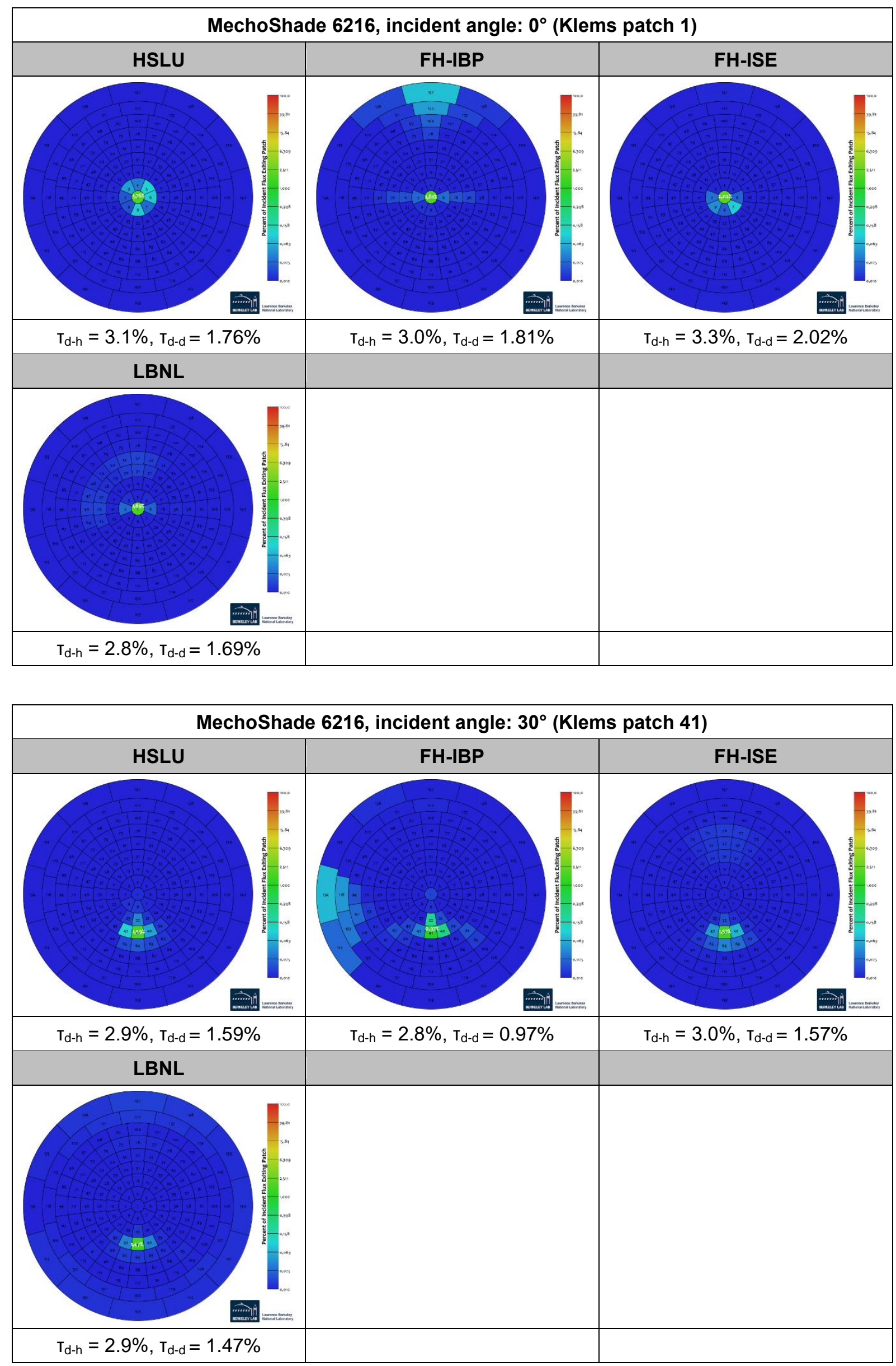

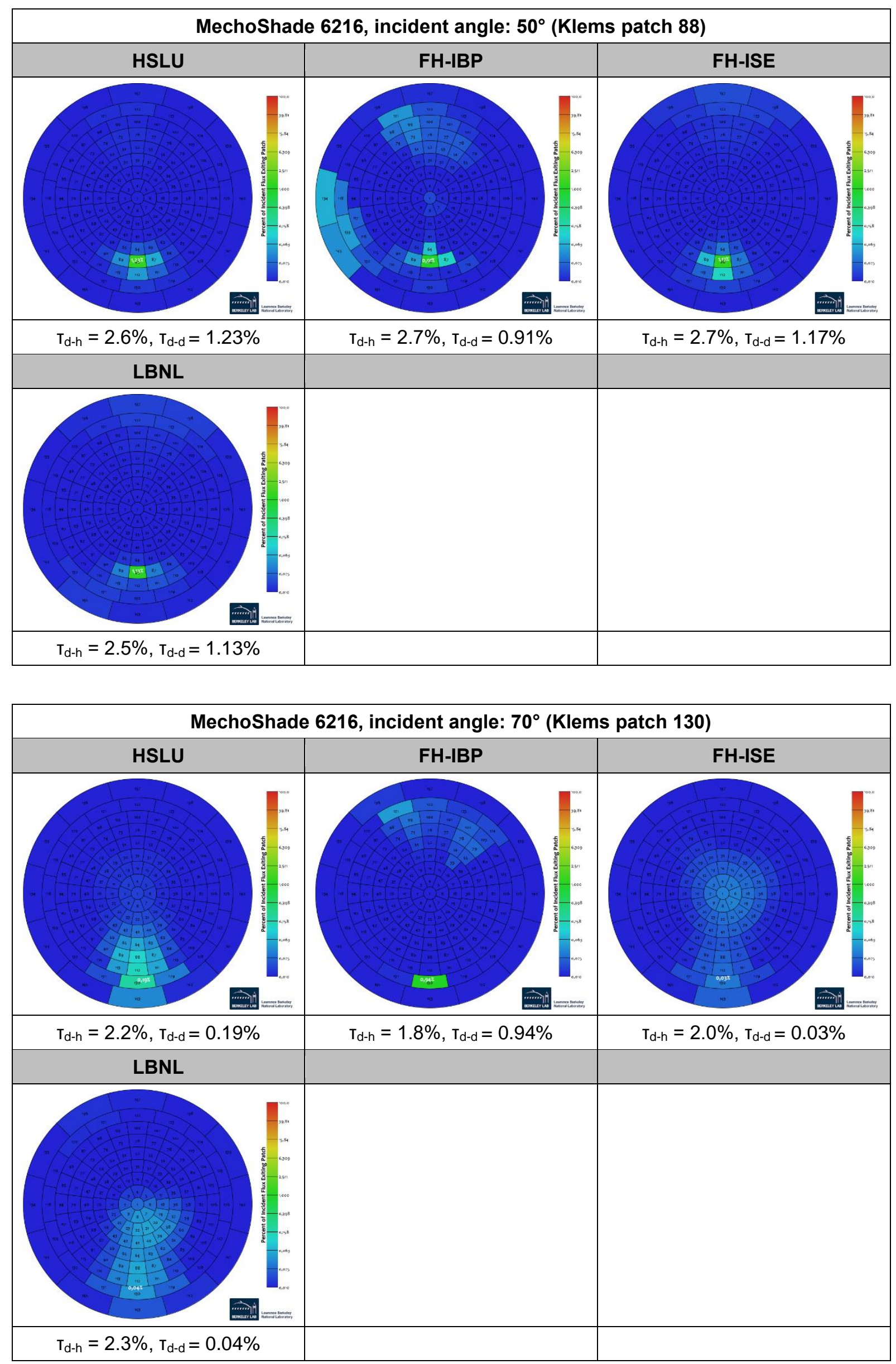


\subsection{Point-in-time simulations}

The point-in-time renderings were performed using a virtual model of the Bartenbach R\&D office building which is used as a living lab ${ }^{49,50}$. The existing fixed sun shading louvers were removed from the model to allow an evaluation of the different BSDF data sets also for sunny conditions.

The situation represents a sunny day using the Utah sky model for the location of Aldrans near Innsbruck, Austria, at $47.3^{\circ} \mathrm{N} / 11.4^{\circ} \mathrm{E}$ on $21^{\text {st }}$ of February at $14: 00 \mathrm{CET}$. Additional calculations were performed for $21^{\text {st }}$ of December, January, March, April, May, and June to get a better understanding of the influence of variations in single BSDF values (i.e., single directions), which are especially pronounced in high-resolution data sets. To show the direct effect of the BSDF values, specular sampling was switched off in the simulations, which, in practice, would be used for a smoother appearance of the façade system in the rendering. Figure 45 to Figure 48 and Figure 55 to Figure 58 show the renderings (top) together with falsecolor luminance (lower left) and glare sources identified by evalglare (lower right).

\subsubsection{HELLA ARB 80}

\subsubsection{1 $21^{\text {st }}$ February, 14:00}

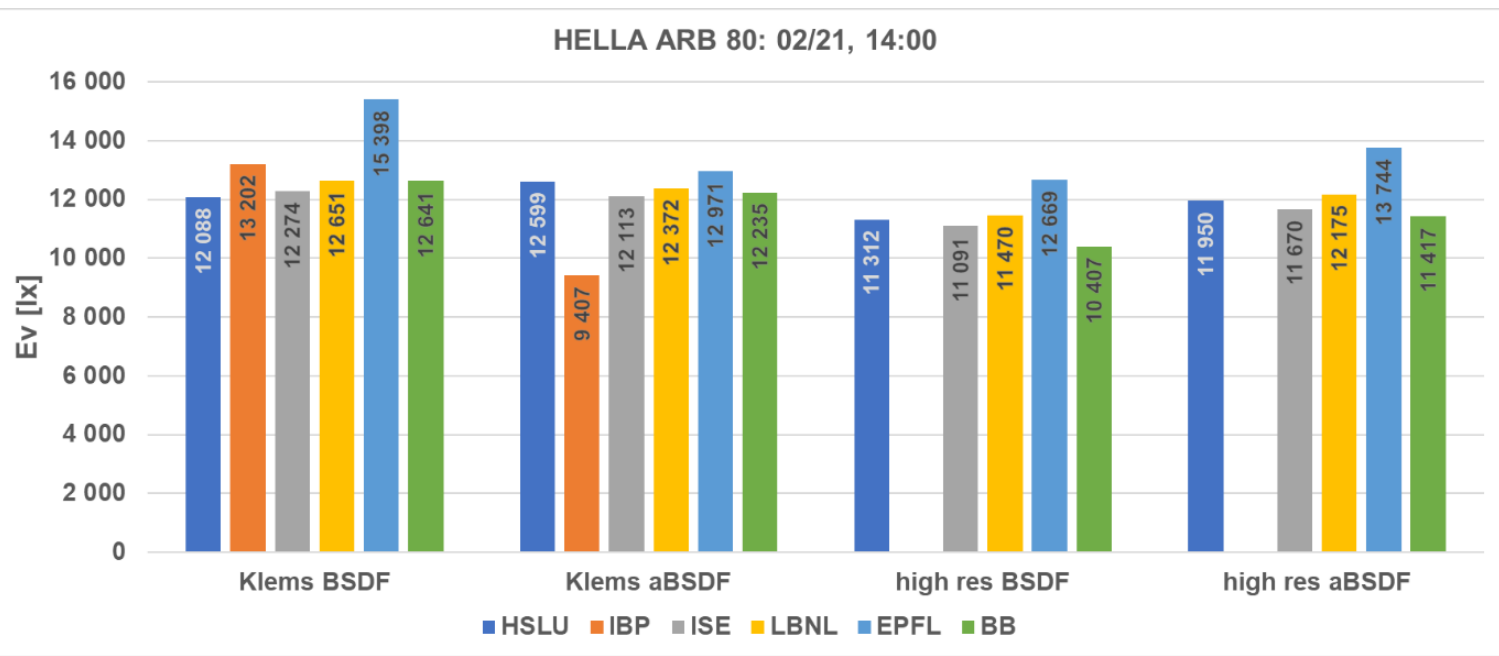

Figure 43: Comparison of Ev values calculated from renderings using the HELLA ARB 80 BSDF data sets in Figure 45 to Figure 48.

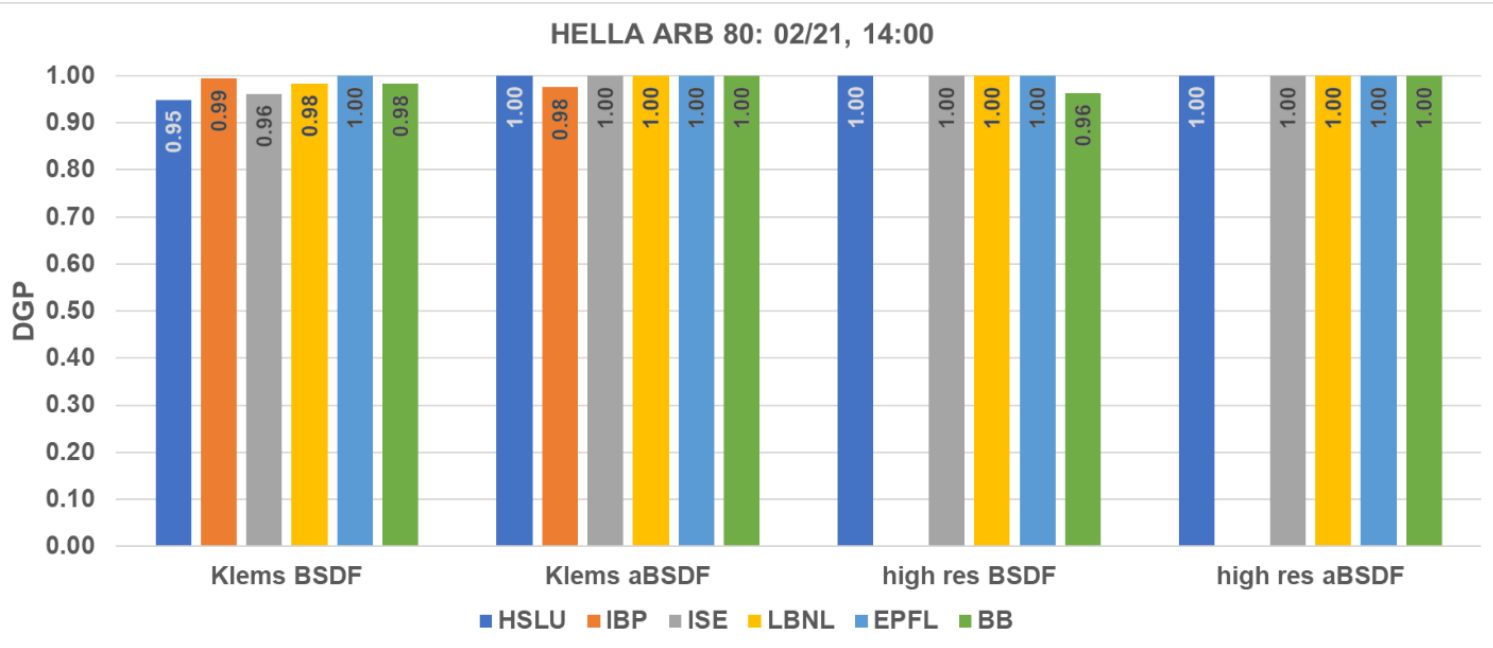

Figure 44: Comparison of DGP values calculated from renderings using the HELLA ARB 80 BSDF data sets in Figure 45 to Figure 48.

49 D. Geisler-Moroder (editor), Workflows and software for the design of integrated lighting solutions. T61.C.1 - A Technical Report of Subtask C, IEA SHC Task 61 / EBC Annex 77, online: https://task61.iea-shc.org/publications, 2019.

50 N. Gentile and W. Osterhaus (editors), Integrating daylighting and lighting in practice, Lessons learned from international case studies. Technical Report of Subtask D, IEA SHC Task 61 / EBC Annex 77, online: https://task61.iea-shc.org/publications, 2021. 

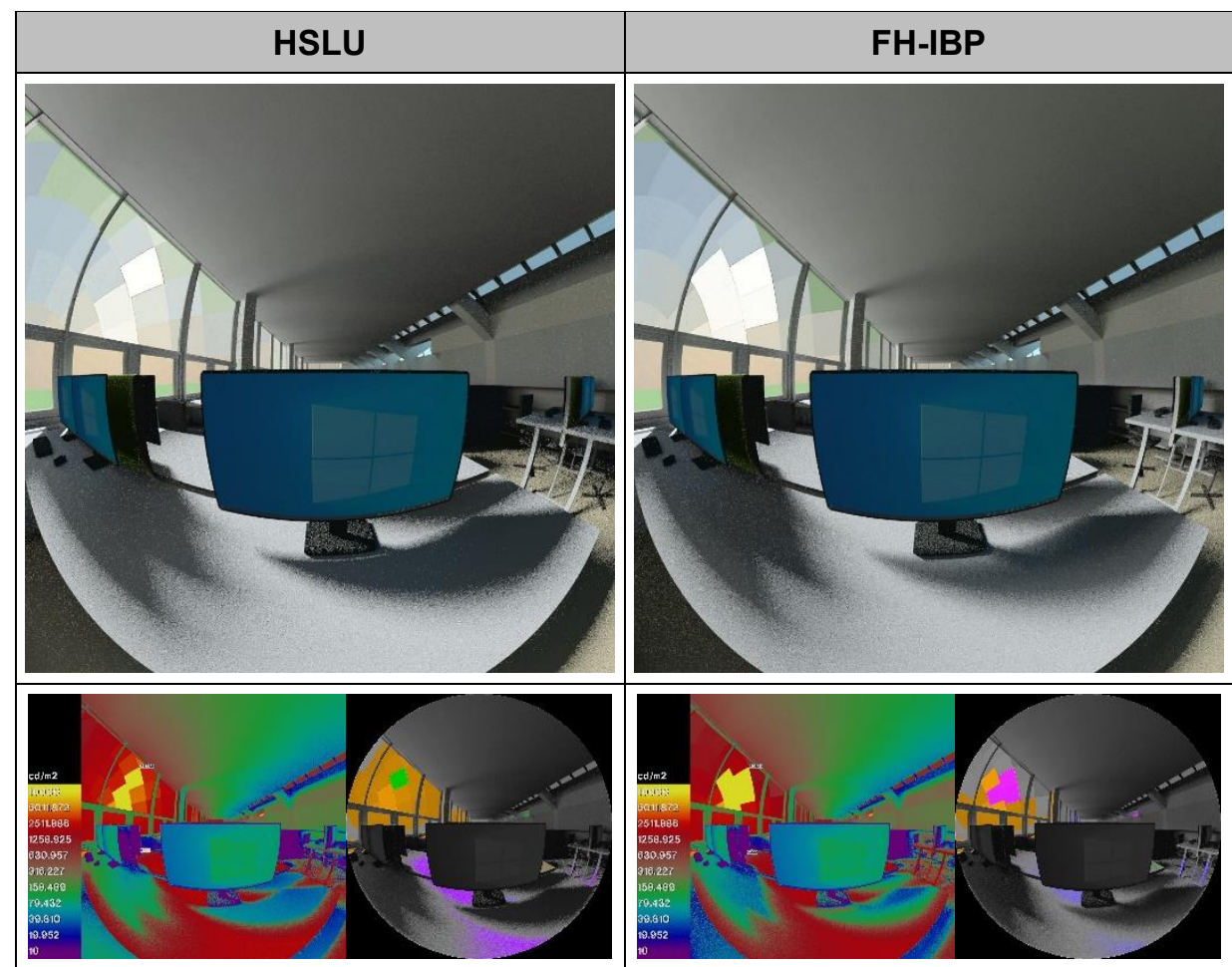

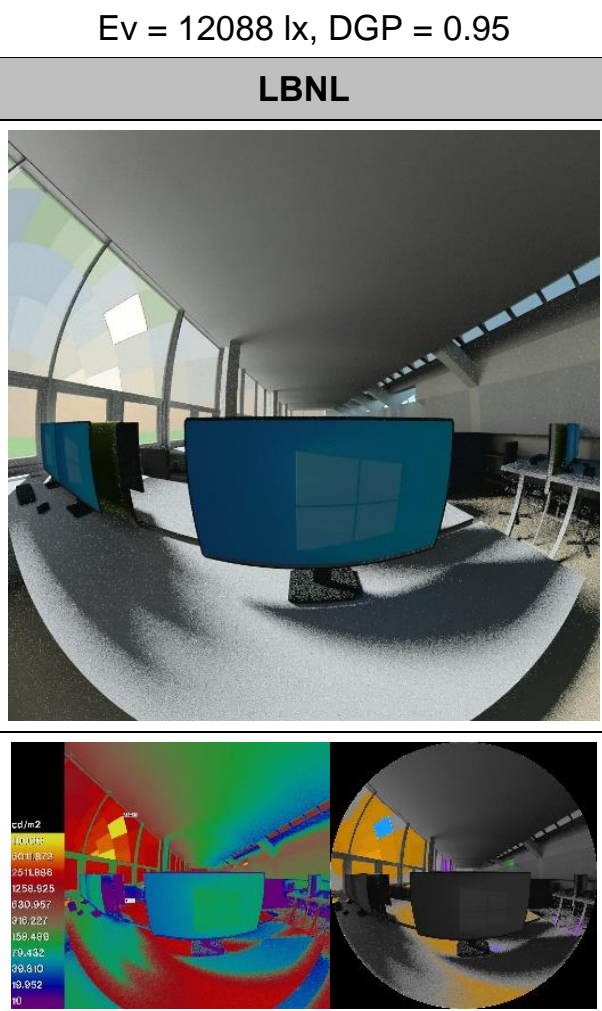

$\mathrm{Ev}=12651 \mathrm{~lx}, \mathrm{DGP}=0.98$

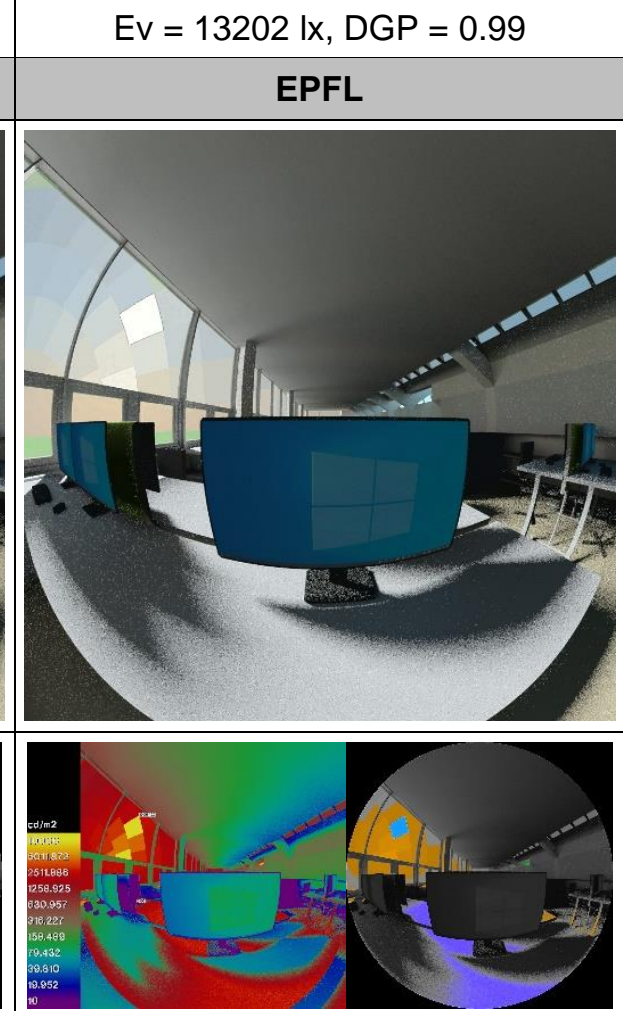

$\mathrm{Ev}=15398 \mathrm{Ix}, \mathrm{DGP}=1.00$
FH-ISE

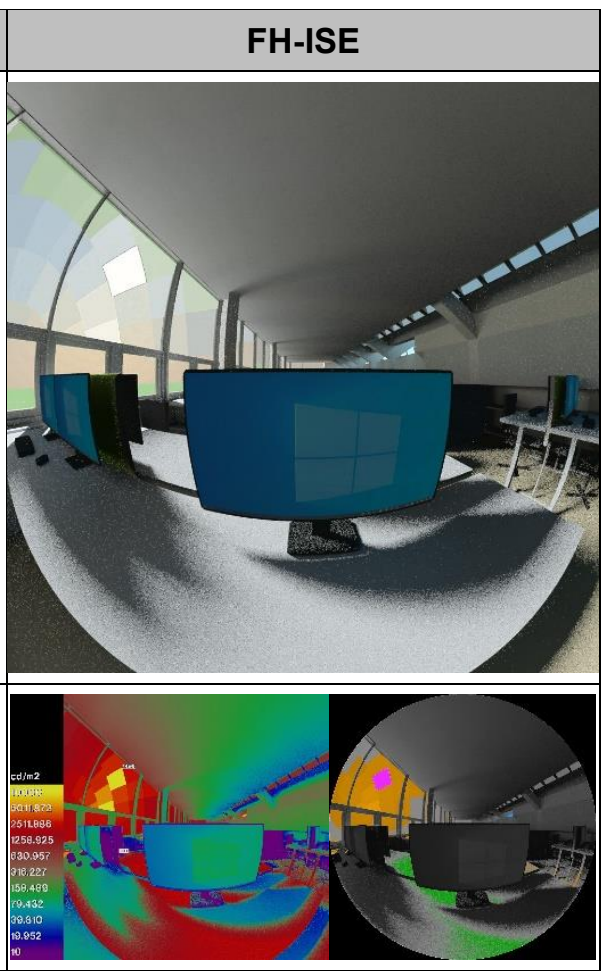

$\mathrm{Ev}=12274 \mathrm{Ix}, \mathrm{DGP}=0.96$

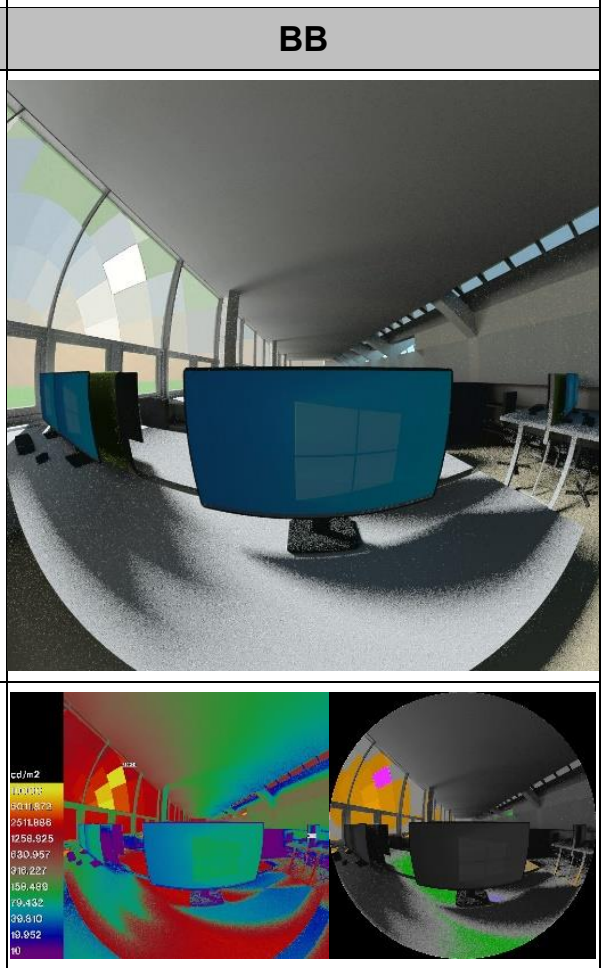

$\mathrm{Ev}=12641 \mathrm{Ix}, \mathrm{DGP}=0.98$

Figure 45: Point-in-time renderings using HELLA ARB 80 BSDF data sets in Klems resolution without peak extraction ("BSDF" material model). 

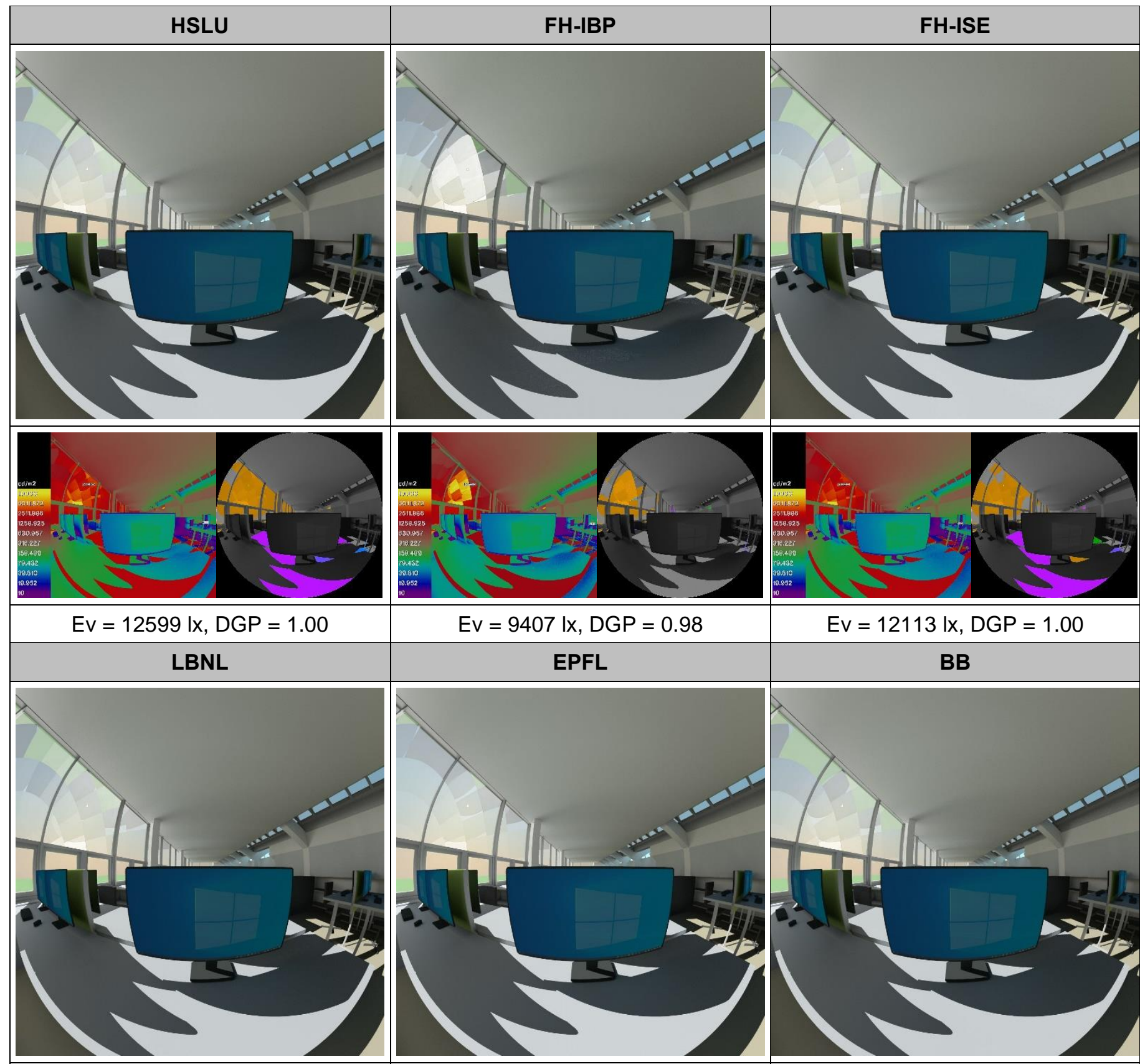

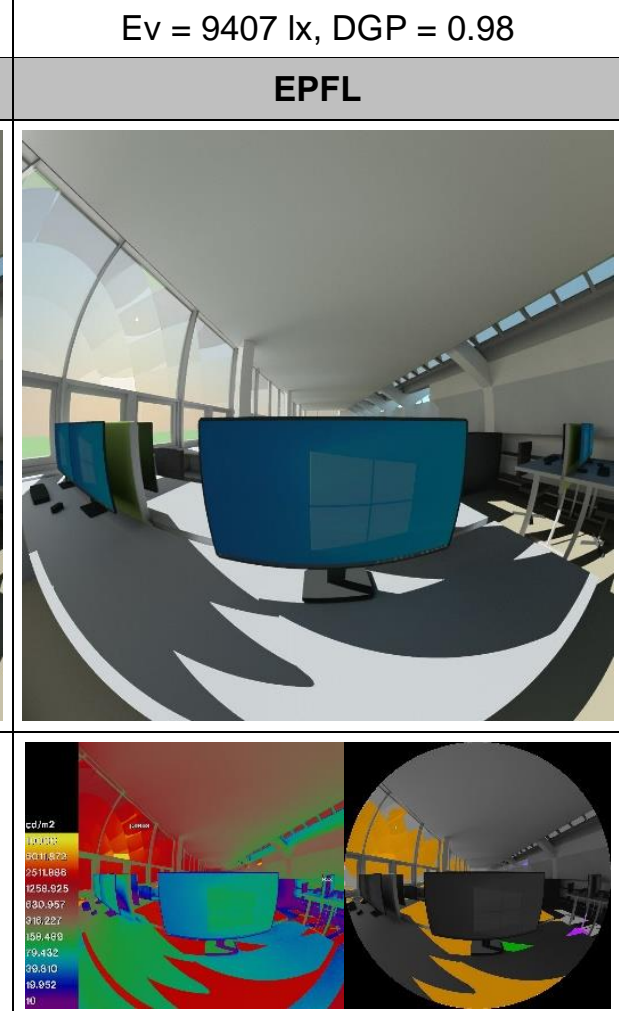

$\mathrm{Ev}=12972 \mathrm{Ix}, \mathrm{DGP}=1.00$
$\mathrm{Ev}=12113 \mathrm{Ix}, \mathrm{DGP}=1.00$

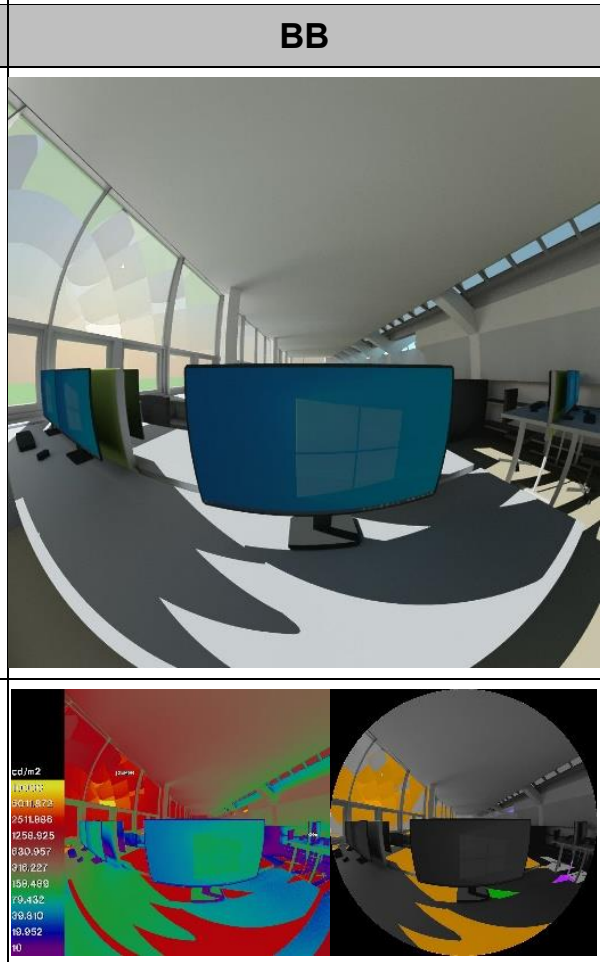

$\mathrm{Ev}=12234 \mathrm{Ix}, \mathrm{DGP}=1.00$

Figure 46: Point-in-time renderings using HELLA ARB 80 BSDF data sets in Klems resolution with peak extraction ("aBSDF" material model). 


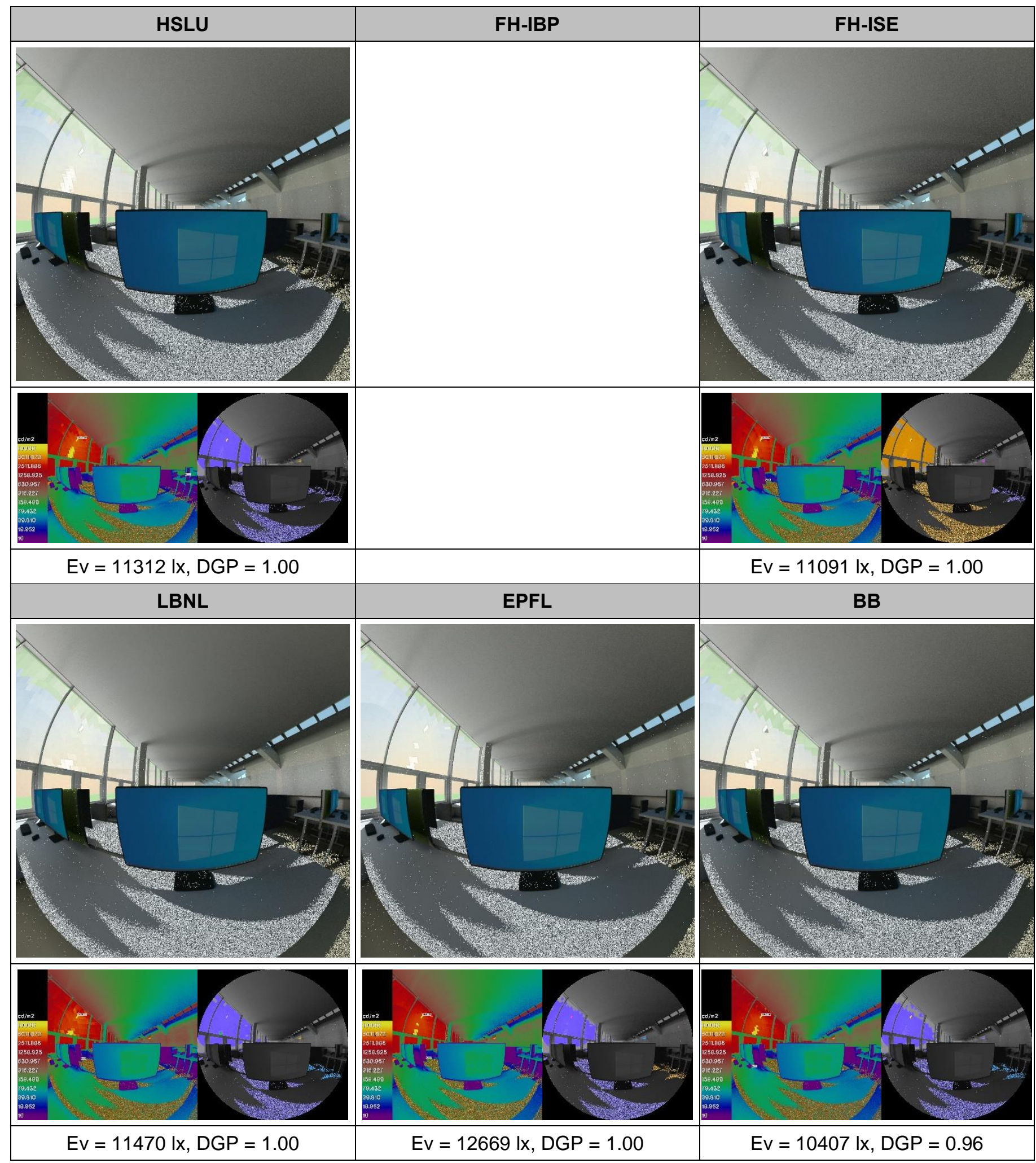

Figure 47: Point-in-time renderings using HELLA ARB 80 BSDF data sets in high resolution (max. 4096x4096) without peak extraction ("BSDF" material model). 

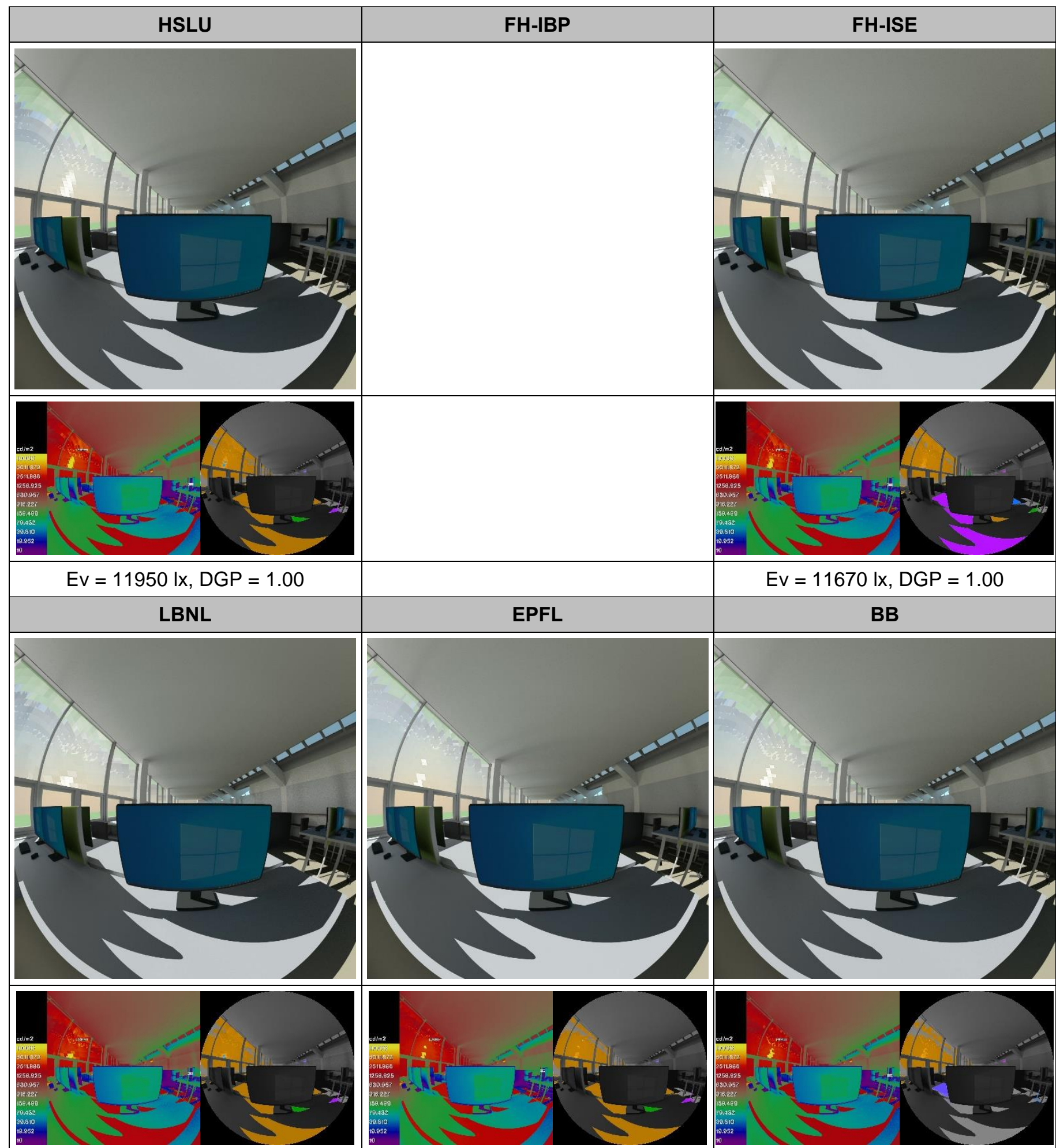

$\mathrm{Ev}=12175 \mathrm{~lx}, \mathrm{DGP}=1.00$

$\mathrm{Ev}=13744 \mathrm{Ix}, \mathrm{DGP}=1.00$

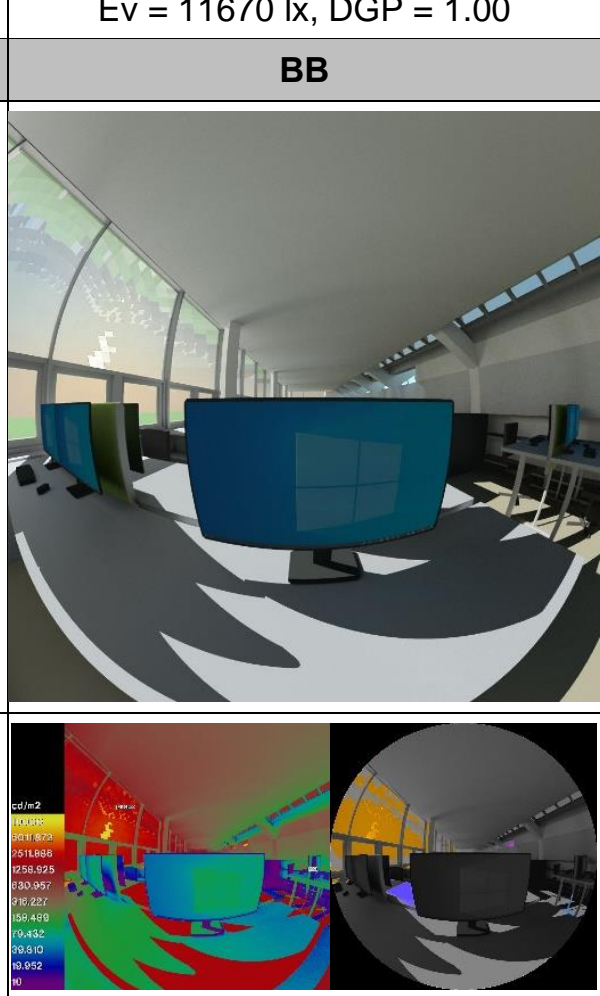

$\mathrm{Ev}=11417 \mathrm{Ix}, \mathrm{DGP}=1.00$

Figure 48: Point-in-time renderings using HELLA ARB 80 BSDF data sets in high resolution (max. 4096x4096) with peak extraction ("aBSDF" material model). 


\subsubsection{2 $21^{\text {st }}$ of each month, 14:00}

To avoid possible random results in the differences caused by a specific solar position and thus BSDF direction, we investigated additional days, equivalent to other solar positions. The figures below show the results for vertical illuminances (Figure 49 and Figure 50) and DGP values (Figure 51 and Figure 52) evaluated from the respective renderings for the 21st of each month in the first half of the year. Both results - Ev and DGP - show good agreement between the different data sets for most situations and suggest that the differences in section 3.5.1.1 for 21st February are caused by local deviations in the area of the underlying data sets responsible for the direct sun at this time.

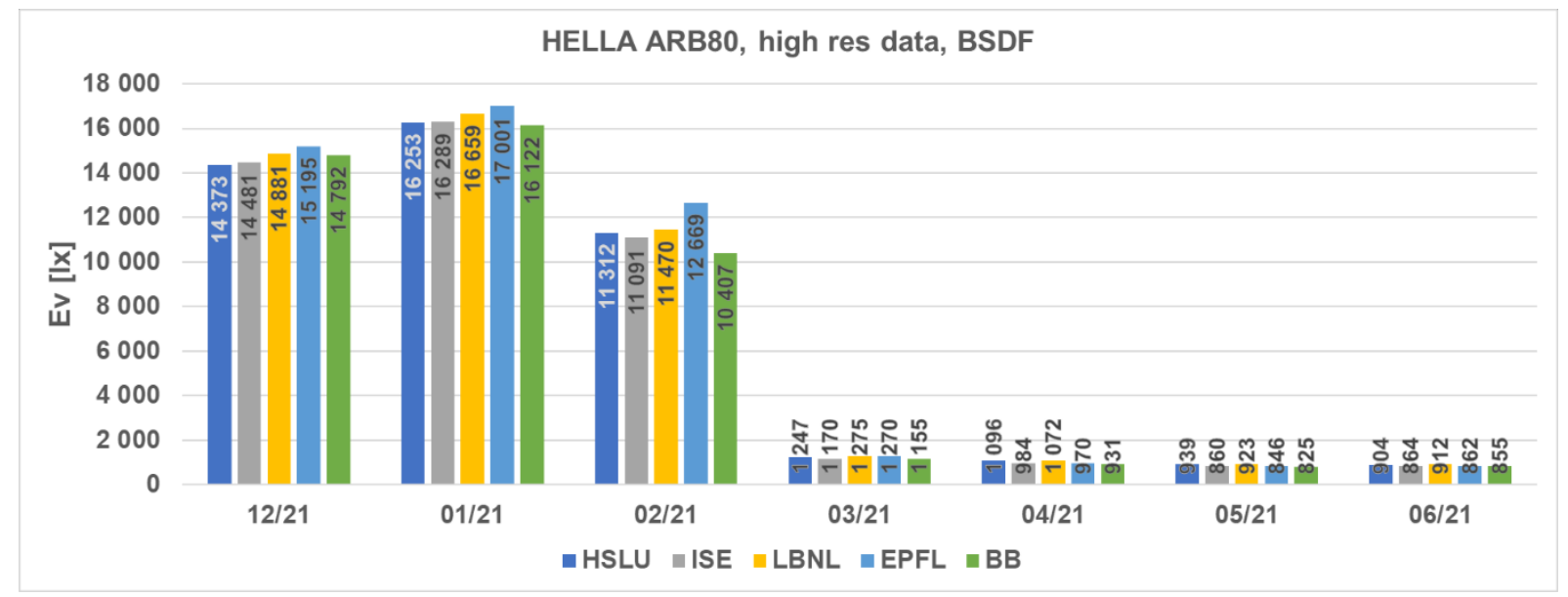

Figure 49: Comparison of Ev values calculated from renderings using the HELLA ARB80 high resolution BSDF data sets without peak extraction ("BSDF" material model).

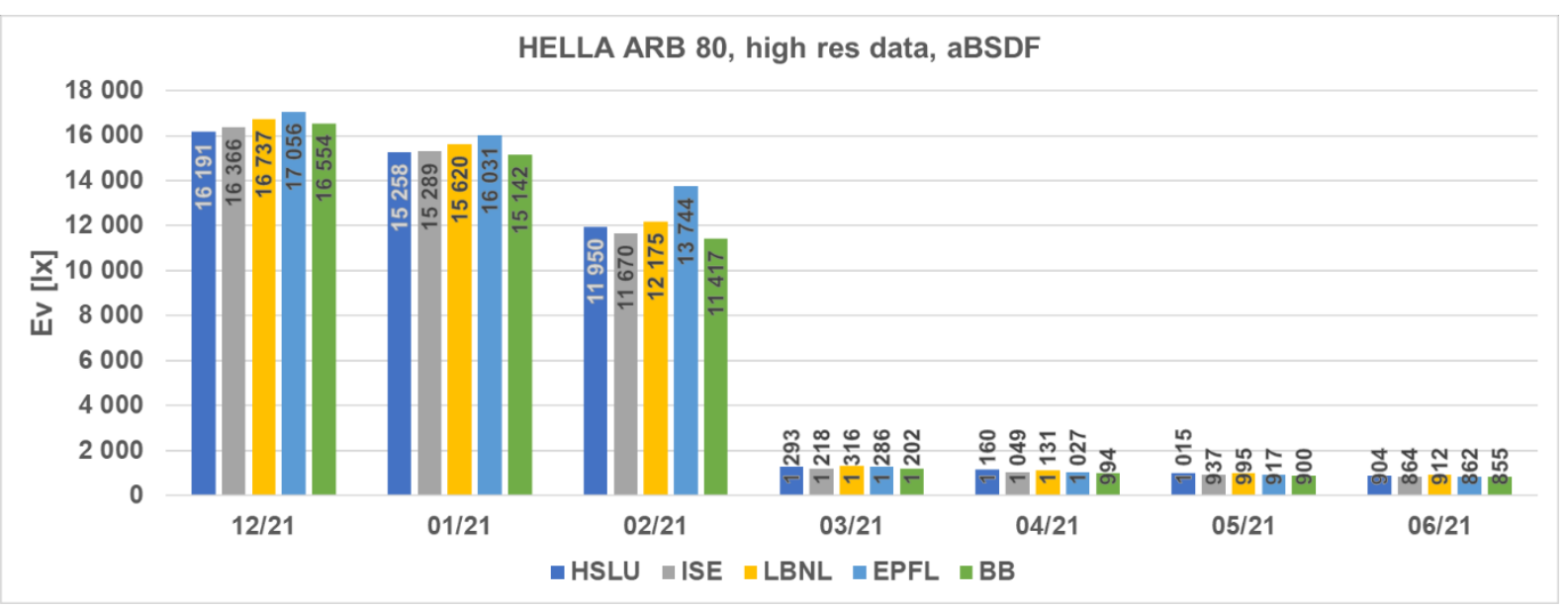

Figure 50: Comparison of Ev values calculated from renderings using the HELLA ARB80 high resolution BSDF data sets with peak extraction ("aBSDF" material model). 


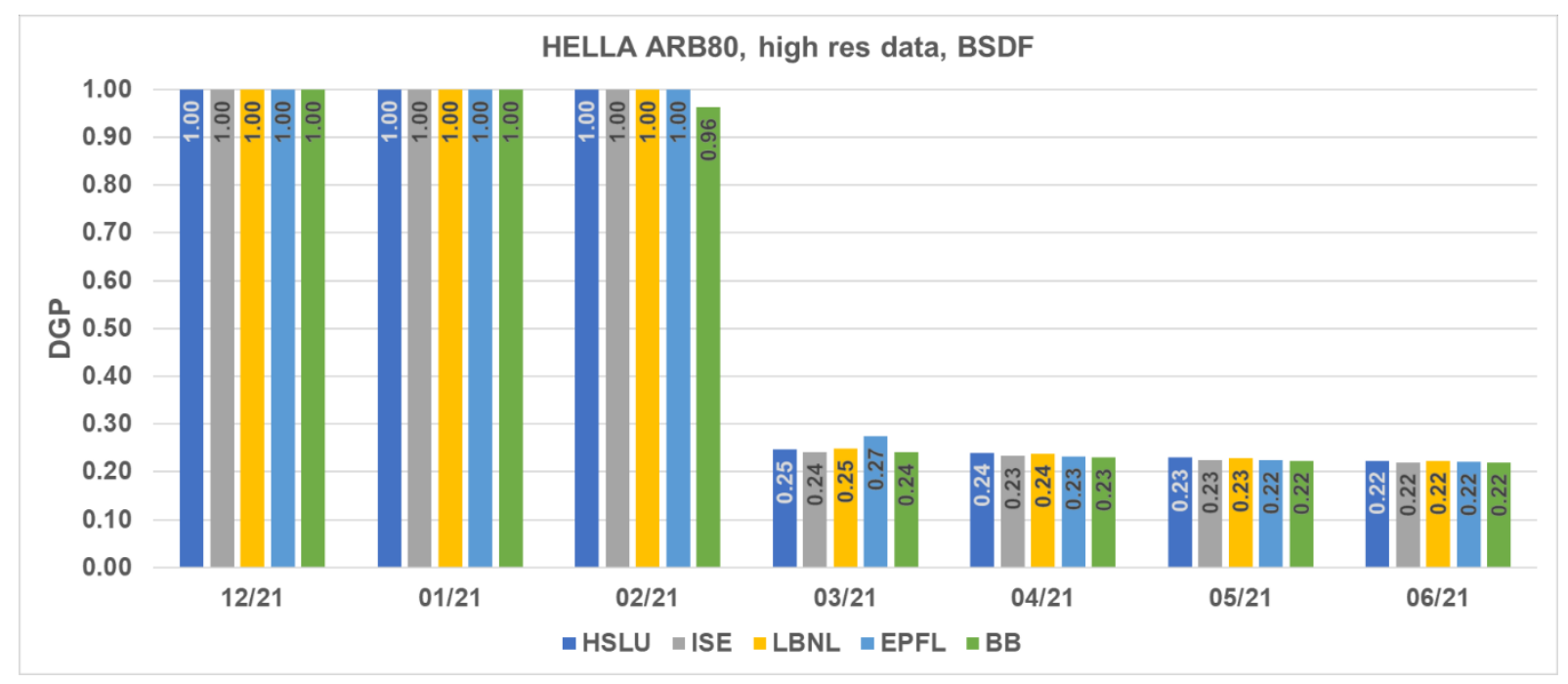

Figure 51: Comparison of DGP values calculated from renderings using the HELLA ARB80 high resolution BSDF data sets without peak extraction ("BSDF" material model).

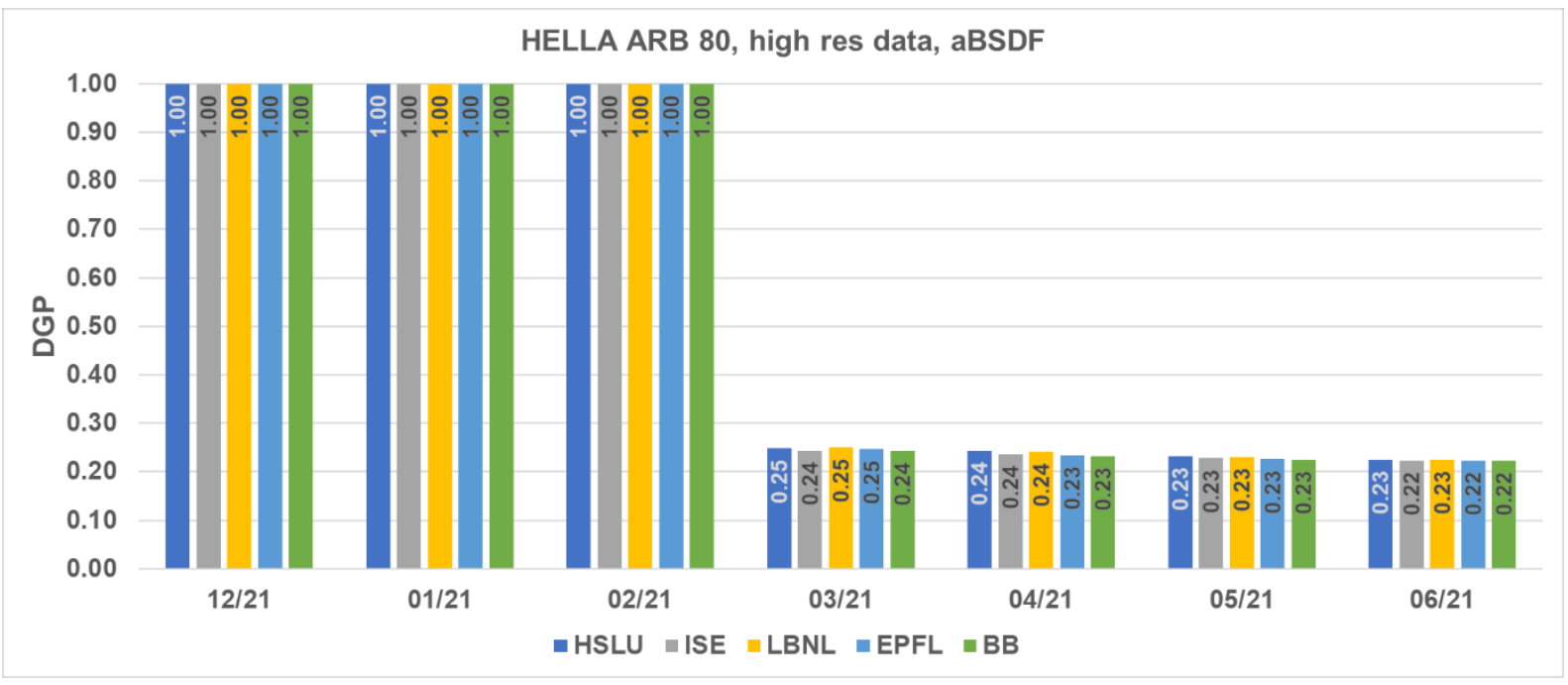

Figure 52: Comparison of DGP values calculated from renderings using the HELLA ARB80 high resolution BSDF data sets without peak extraction ("aBSDF" material model). 


\subsubsection{MechoShade 6216}

\subsubsection{1 $21^{\text {st }}$ February, 14:00}

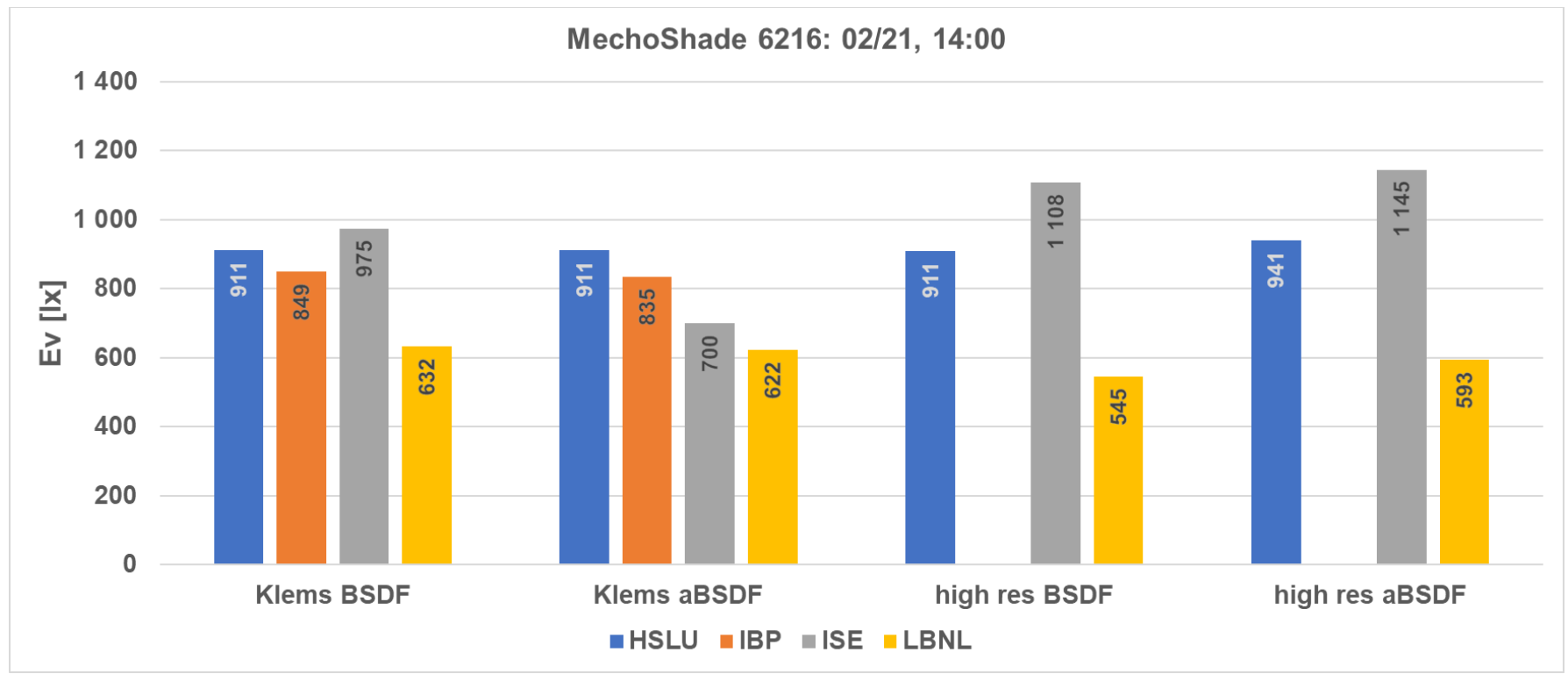

Figure 53: Comparison of Ev values calculated from renderings using the MechoShade 6216 BSDF data sets in Figure 55 to Figure 58.

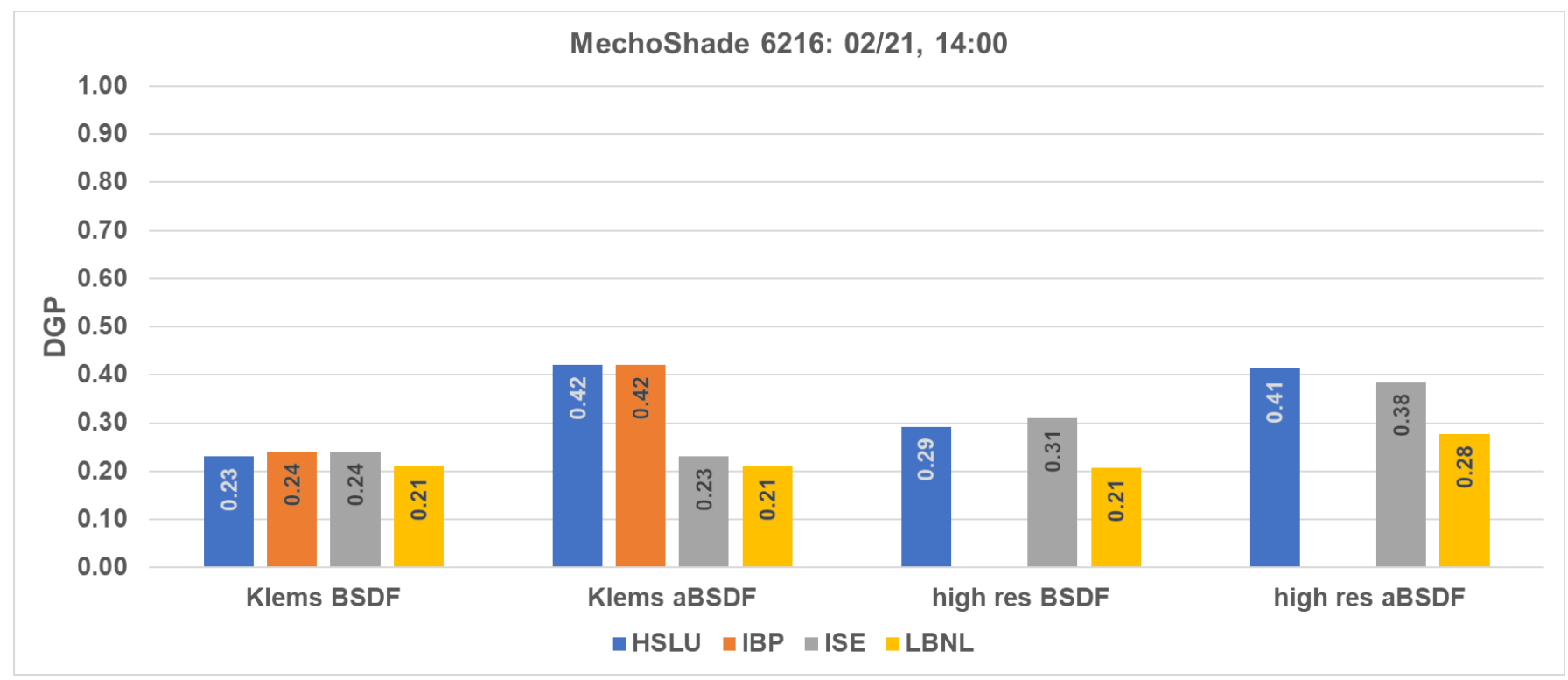

Figure 54: Comparison of DGP values calculated from renderings using the MechoShade 6216 BSDF data sets in Figure 55 to Figure 58. 


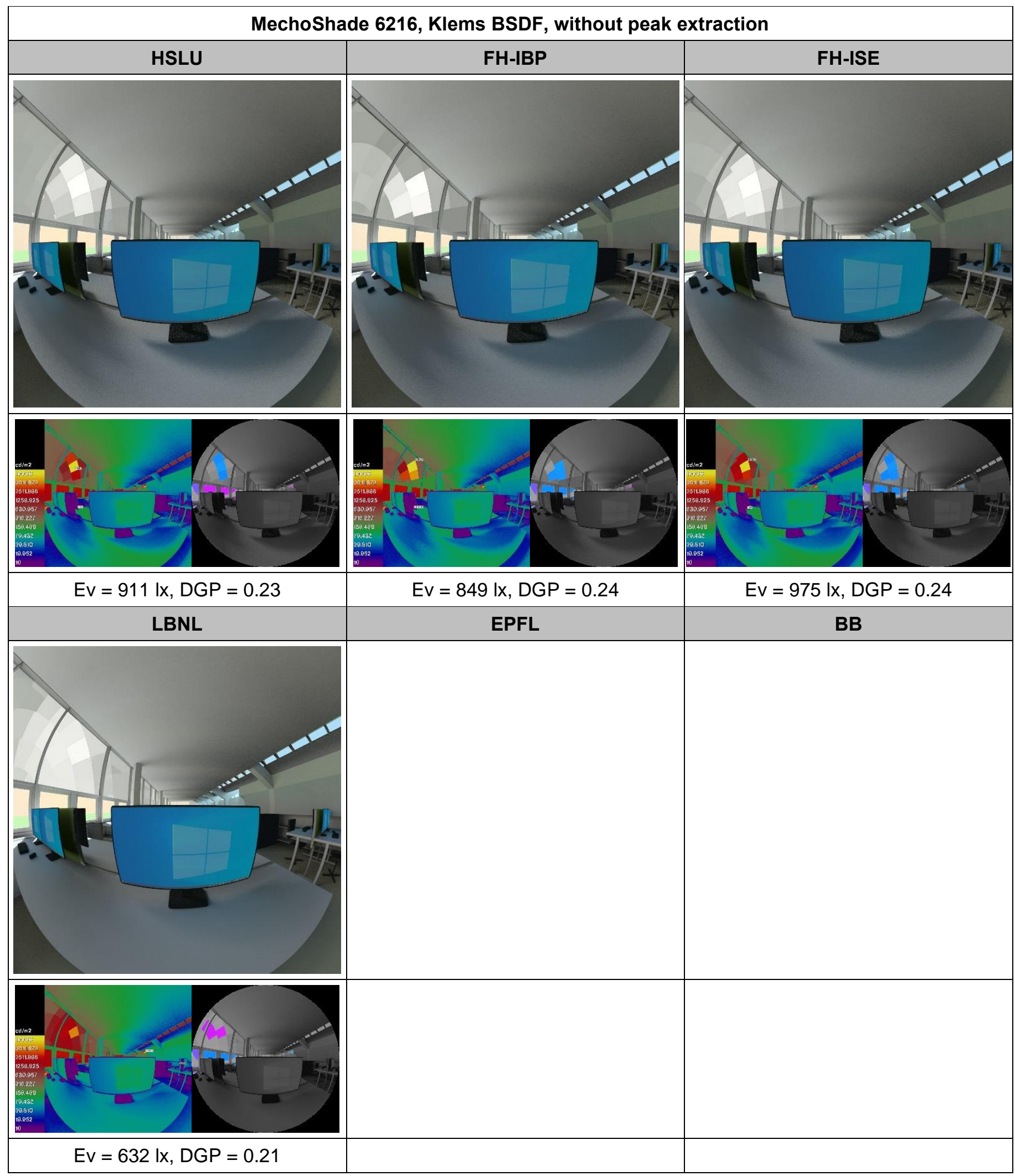

Figure 55: Point-in-time renderings using MechoShade 6216 BSDF data sets in Klems resolution without peak extraction ("BSDF" material model). 


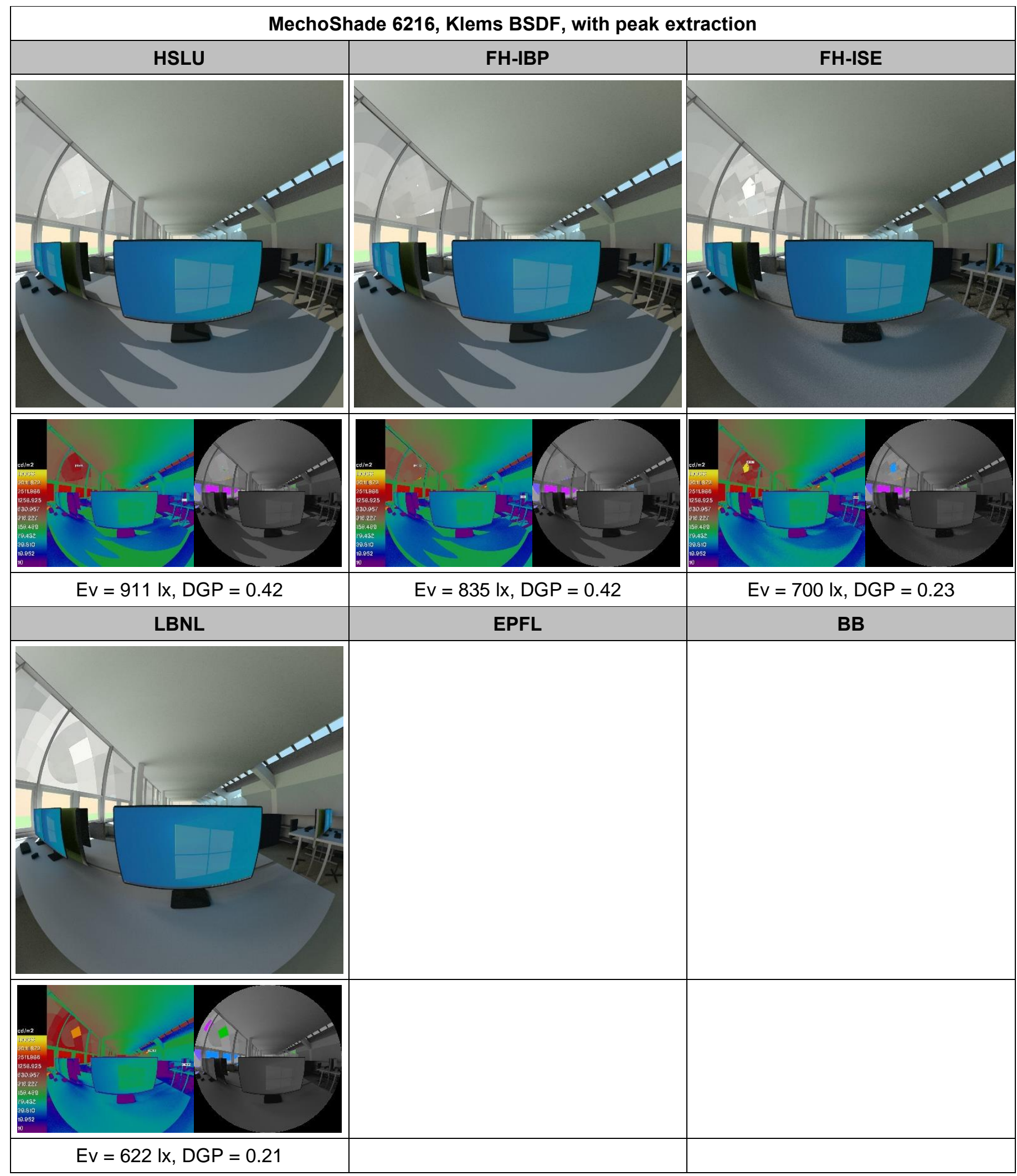

Figure 56: Point-in-time renderings using MechoShade 6216 BSDF data sets in Klems resolution with peak extraction ("aBSDF" material model). 


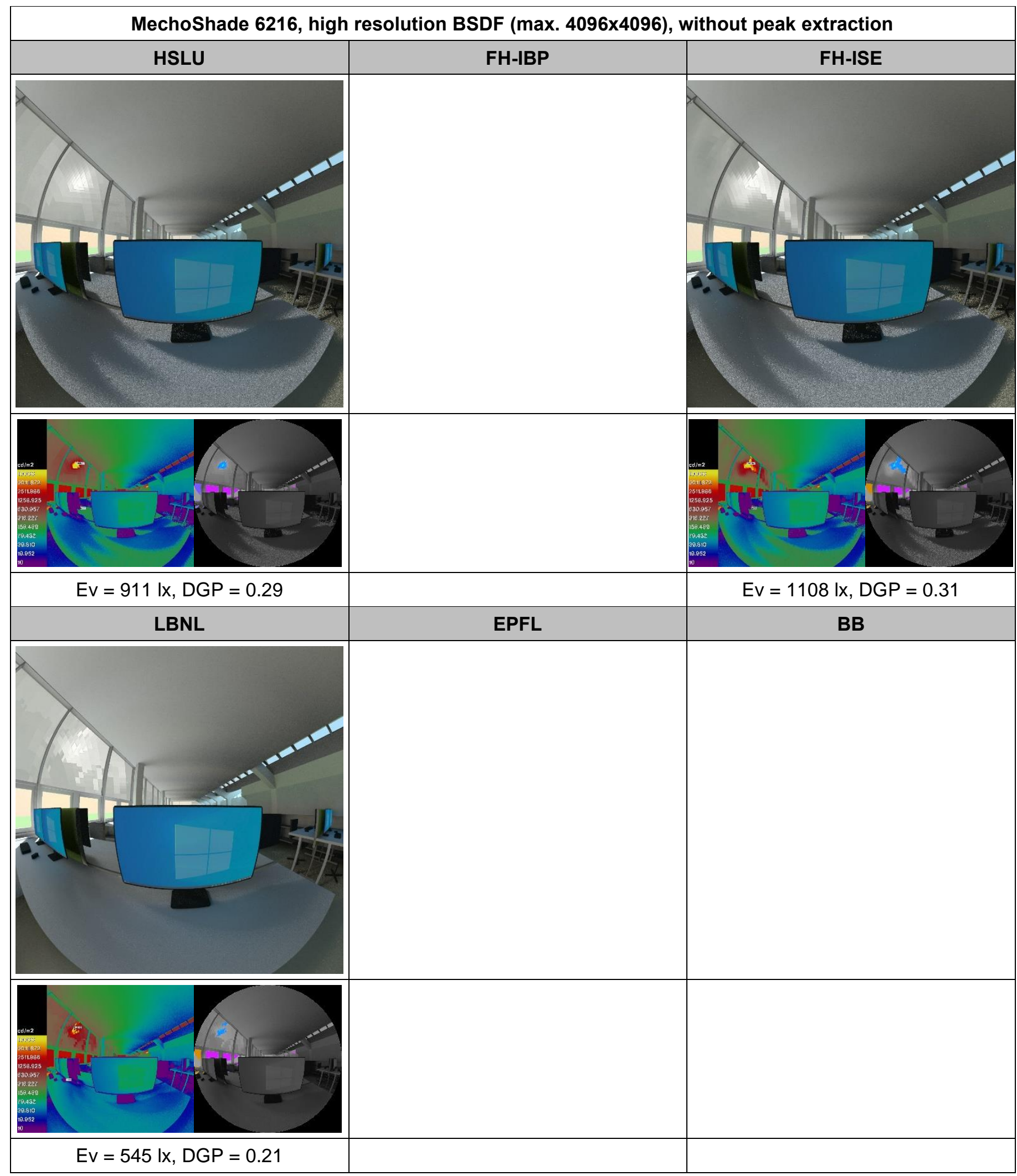

Figure 57: Point-in-time renderings using MechoShade 6216 BSDF data sets in high resolution (max. 4096x4096) without peak extraction ("BSDF" material model). 


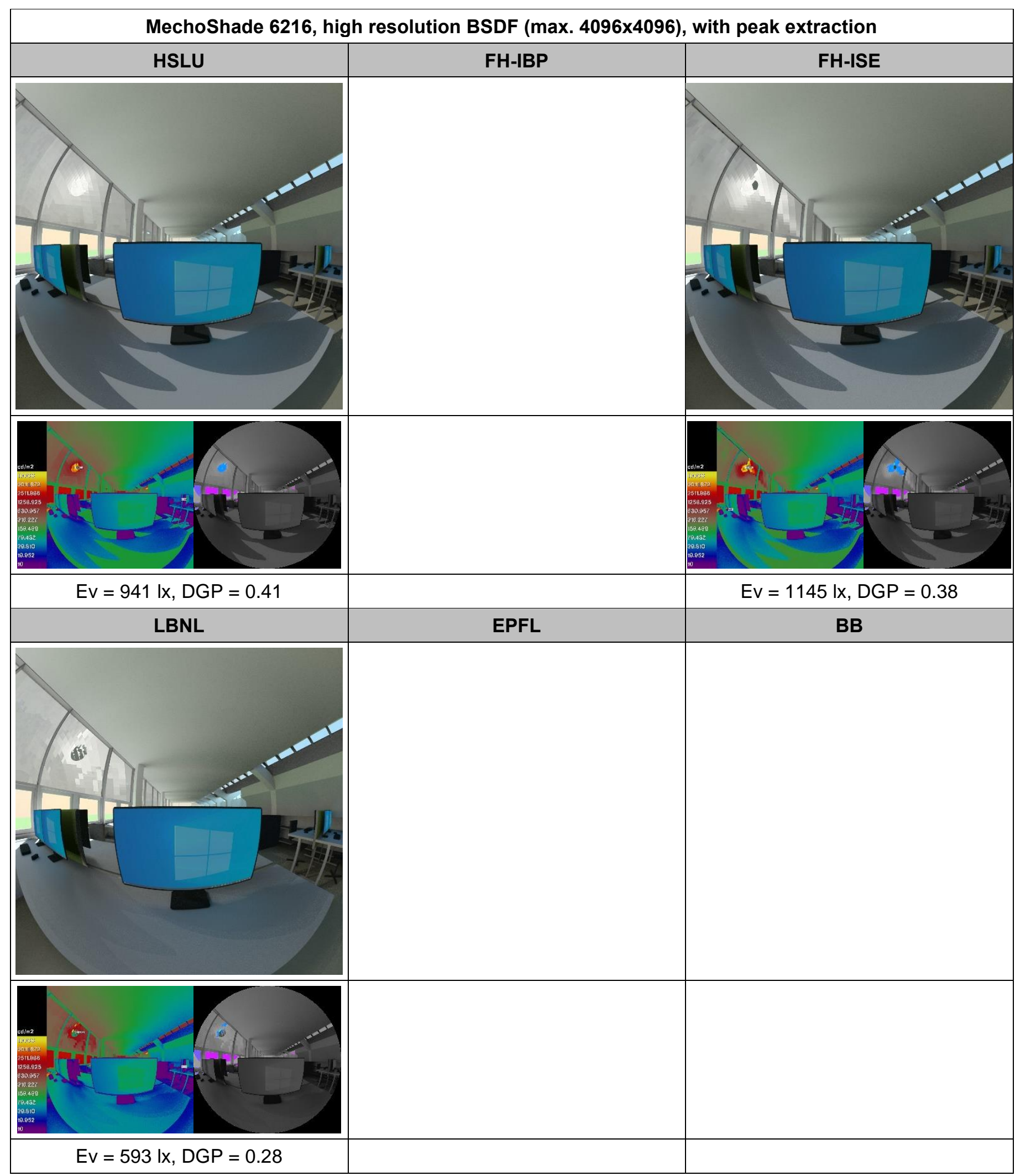

Figure 58: Point-in-time renderings using MechoShade 6216 BSDF data sets in high resolution (max. 4096x4096) with peak extraction ("aBSDF" material model). 


\subsubsection{2 $21^{\text {st }}$ of each month, 14:00}

The relatively high deviations in the simulations for $21^{\text {st }}$ February, 14:00, are due to the fact that the sun position is near the cut-off of the fabric. Around the cut-off area, not only the measurement uncertainty is the highest, but also the peak extraction algorithm (aBSDF material vs. BSDF material) jumps between extracting and not extracting the solar peak. This prompted us to investigate other days and thus solar positions again. The figures below show the results for vertical illuminances (Figure 59 and Figure 60) and DGP values (Figure 61 and Figure 62) evaluated from the respective renderings for the $21^{\text {st }}$ of each month in the first half of the year. Also here, both results - Ev and DGP - show good agreement between the different data sets for most situations and suggest that the differences in section 3.5.2.1 for $21^{\text {st }}$ February are caused by relatively large, local deviations in the area of the underlying data sets responsible for the direct sun at this time.

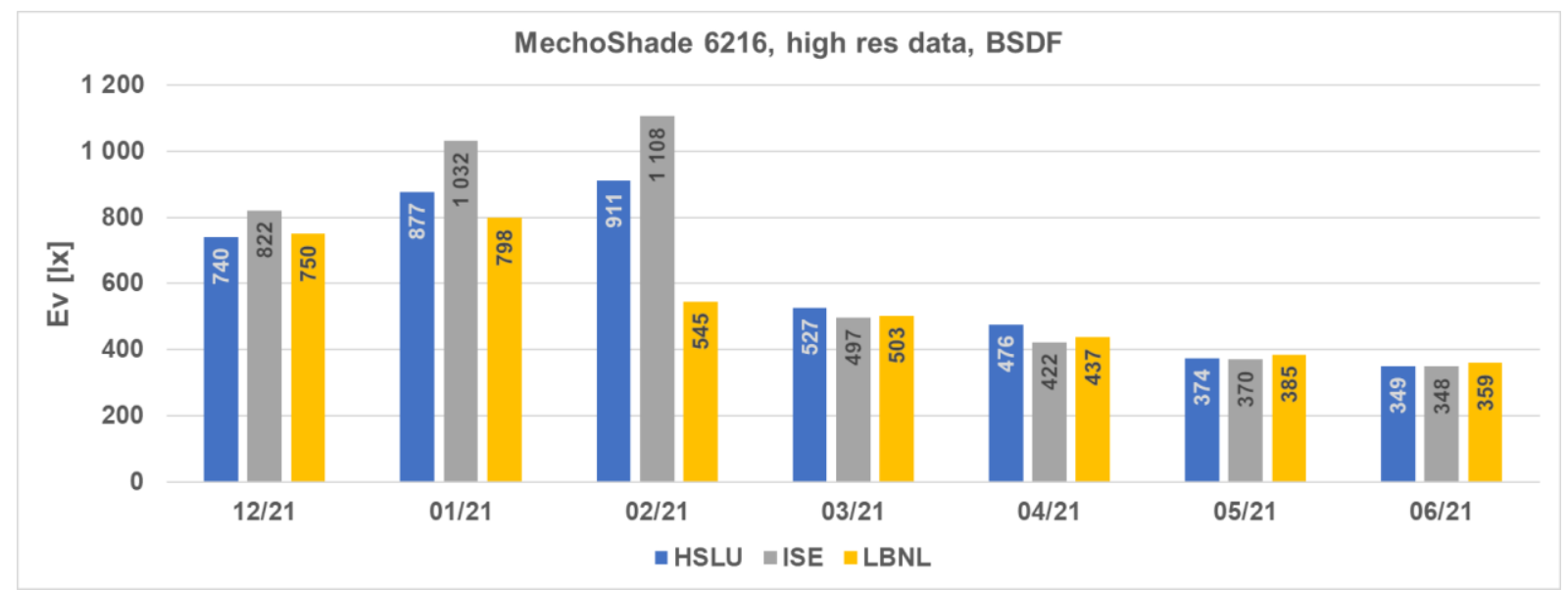

Figure 59: Comparison of Ev values calculated from renderings using the MechoShade 6216 high resolution BSDF data sets without peak extraction ("BSDF" material model).

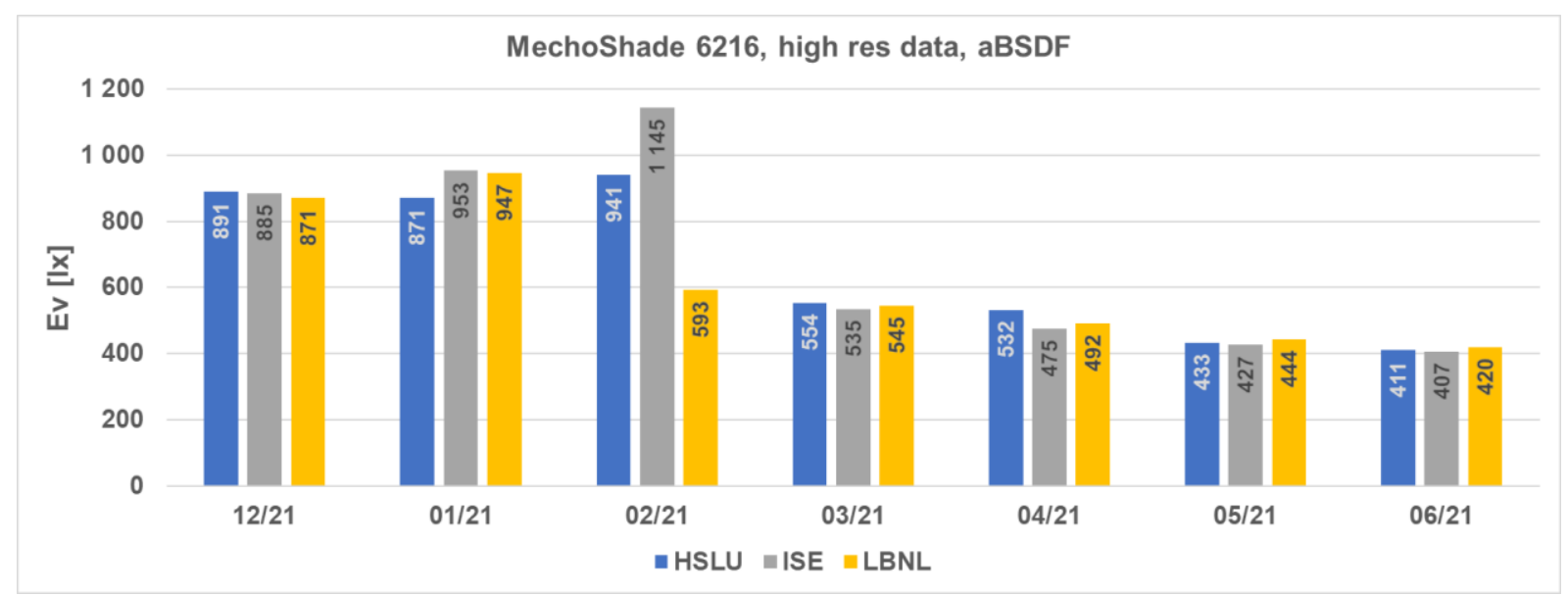

Figure 60: Comparison of Ev values calculated from renderings using the MechoShade 6216 high resolution BSDF data sets with peak extraction ("aBSDF" material model). 


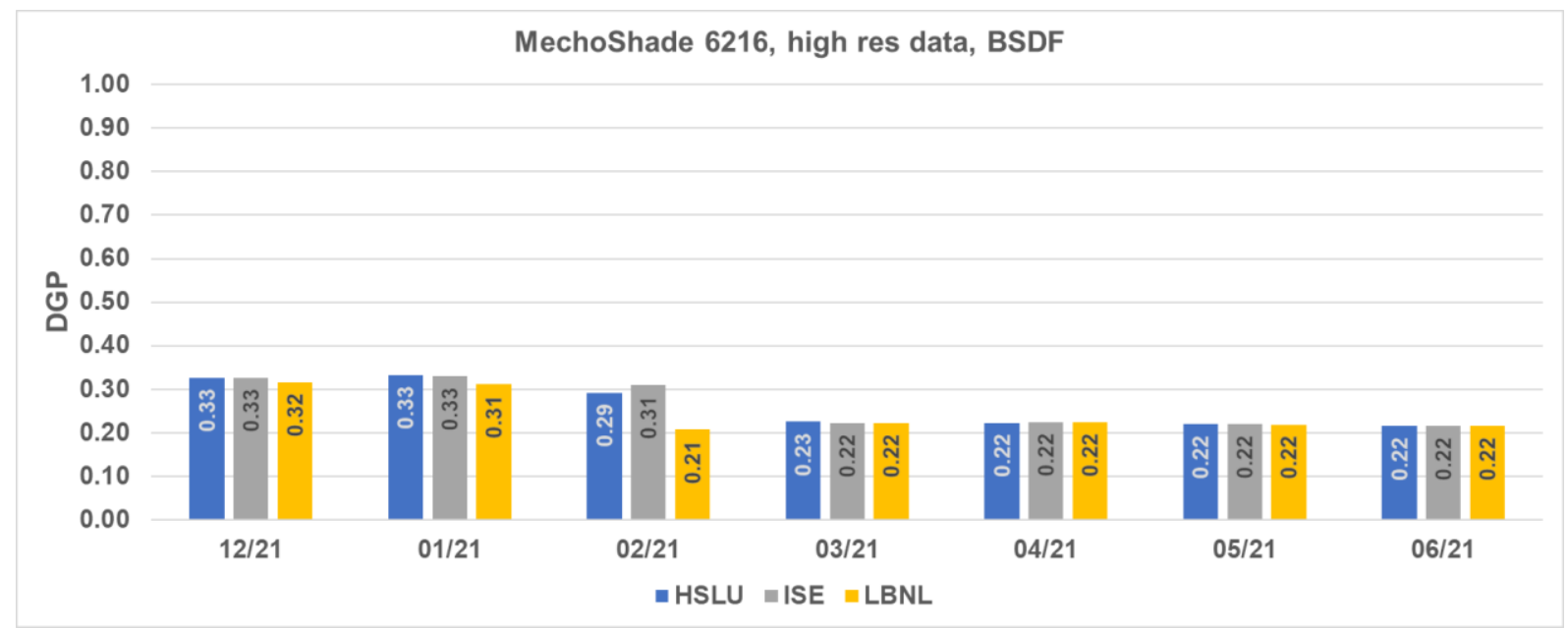

Figure 61: Comparison of DGP values calculated from renderings using the MechoShade 6216 high resolution BSDF data sets without peak extraction ("BSDF" material model).

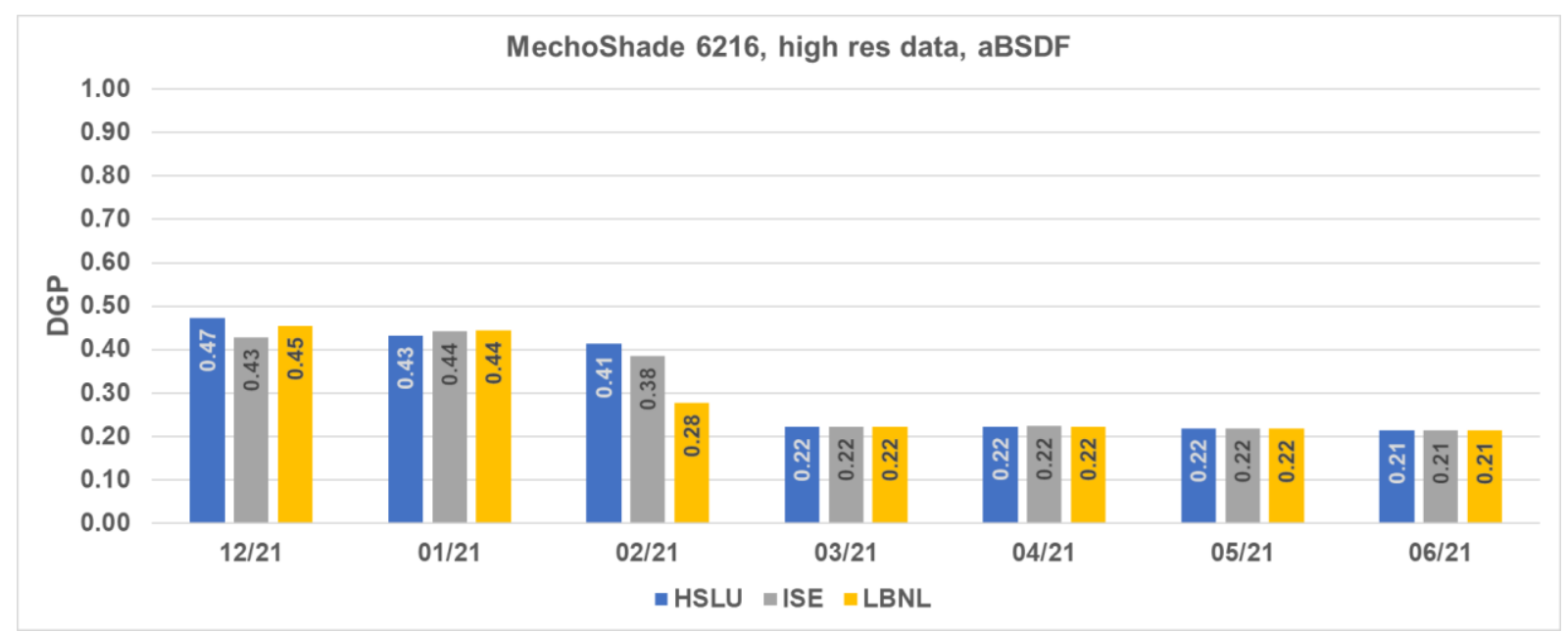

Figure 62: Comparison of DGP values calculated from renderings using the MechoShade 6216 high resolution BSDF data sets with peak extraction ("aBSDF" material model). 


\subsection{Annual simulations}

The annual simulations were performed using a virtual model of the 71T testbed at LBNL in Berkeley, CA, USA. The TMY3 Energy Plus weather data set from Oakland Airport at $37.72^{\circ} \mathrm{N} / 122.22^{\circ} \mathrm{W}$ was used as input to generate sky distributions using the Perez model.
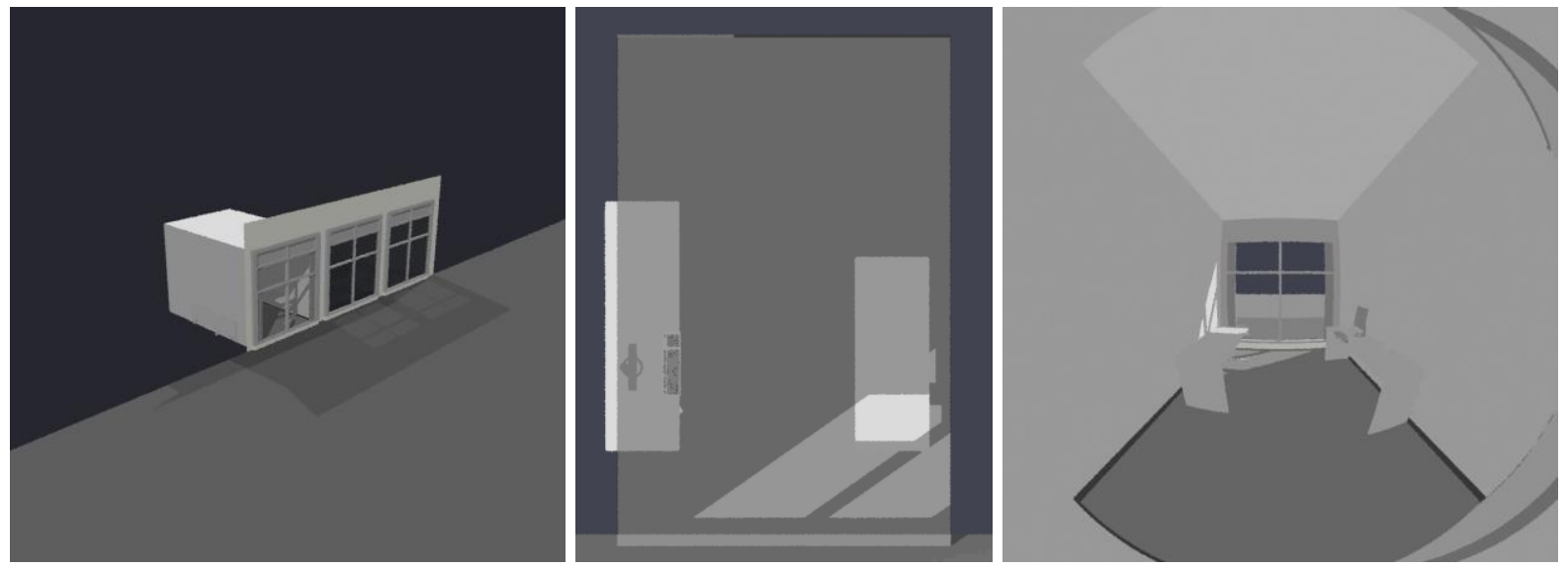

Figure 63: Simulation model of the 71T testbed at LBNL in Berkeley, CA, USA.
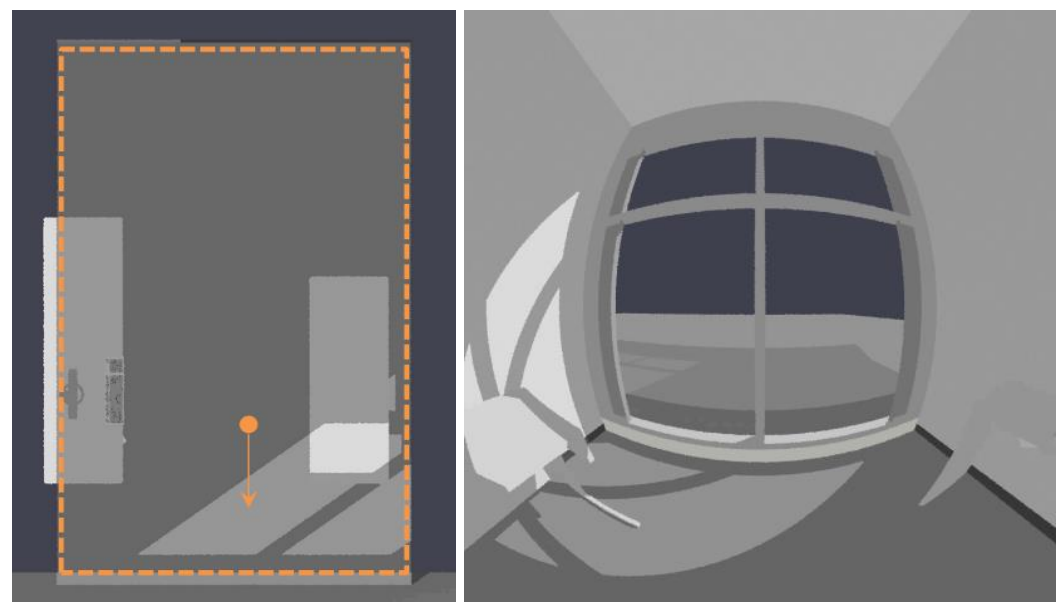

Figure 64: Workplane area and position of virtual camera (left), fisheye view from camera position (right).

The following annual simulations were performed with all available BSDF data sets via three-phasemethod (3PM) and five-phase-method (5PM) calculations:

- Daylight autonomy based on all daylight hours on the workplane (3PM, Klems BSDF)

- Vertical illuminance at camera position (3PM, Klems BSDF)

- DGP and DGI glare index calculation (5PM, Klems and high resolution BSDF, with and without peak extraction), evaluated with evalglare version 2.10 using the default parameter settings. 


\subsubsection{HELLA ARB 80}

\subsubsection{Daylight autonomy}

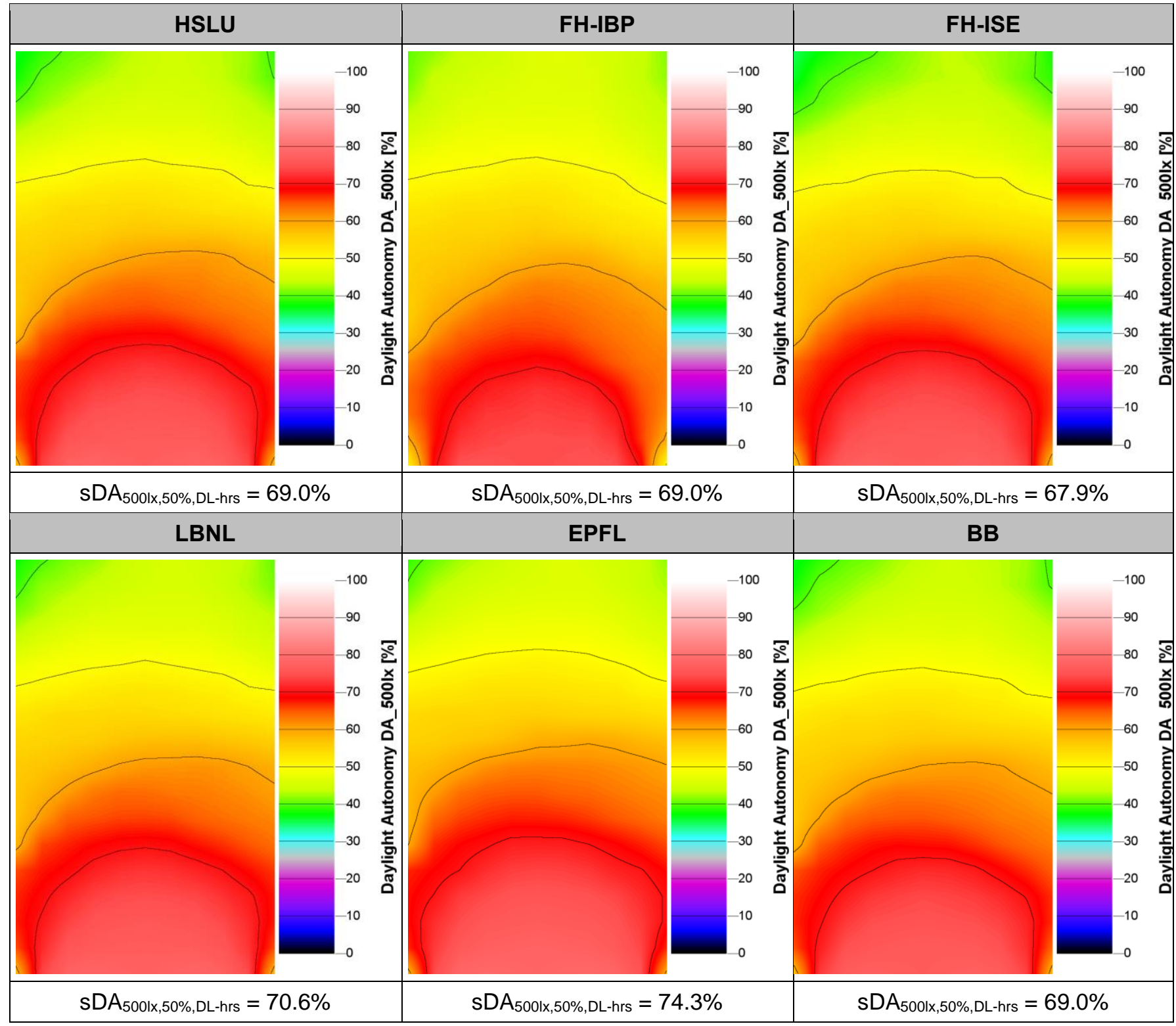

Figure 65: Daylight autonomy (500lx) based on all daylight hours on workplane using HELLA ARB 80 BSDF data sets in Klems resolution in 3PM calculation. 


\subsubsection{Annual vertical illuminance at camera position}

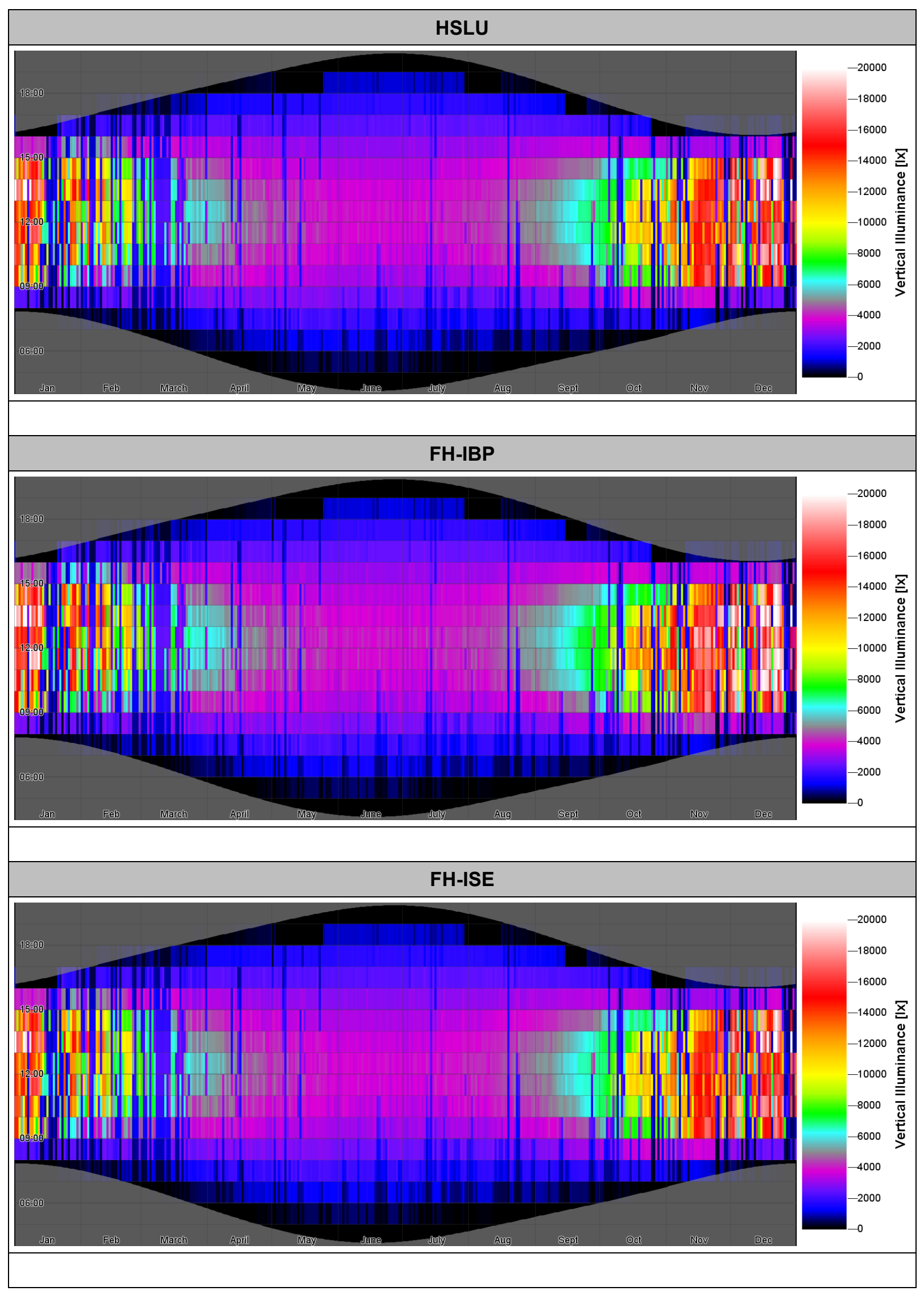

Figure 66: (1/2) Annual vertical illuminance at camera position using HELLA ARB 80 BSDF data sets in Klems resolution in 3PM calculation. 


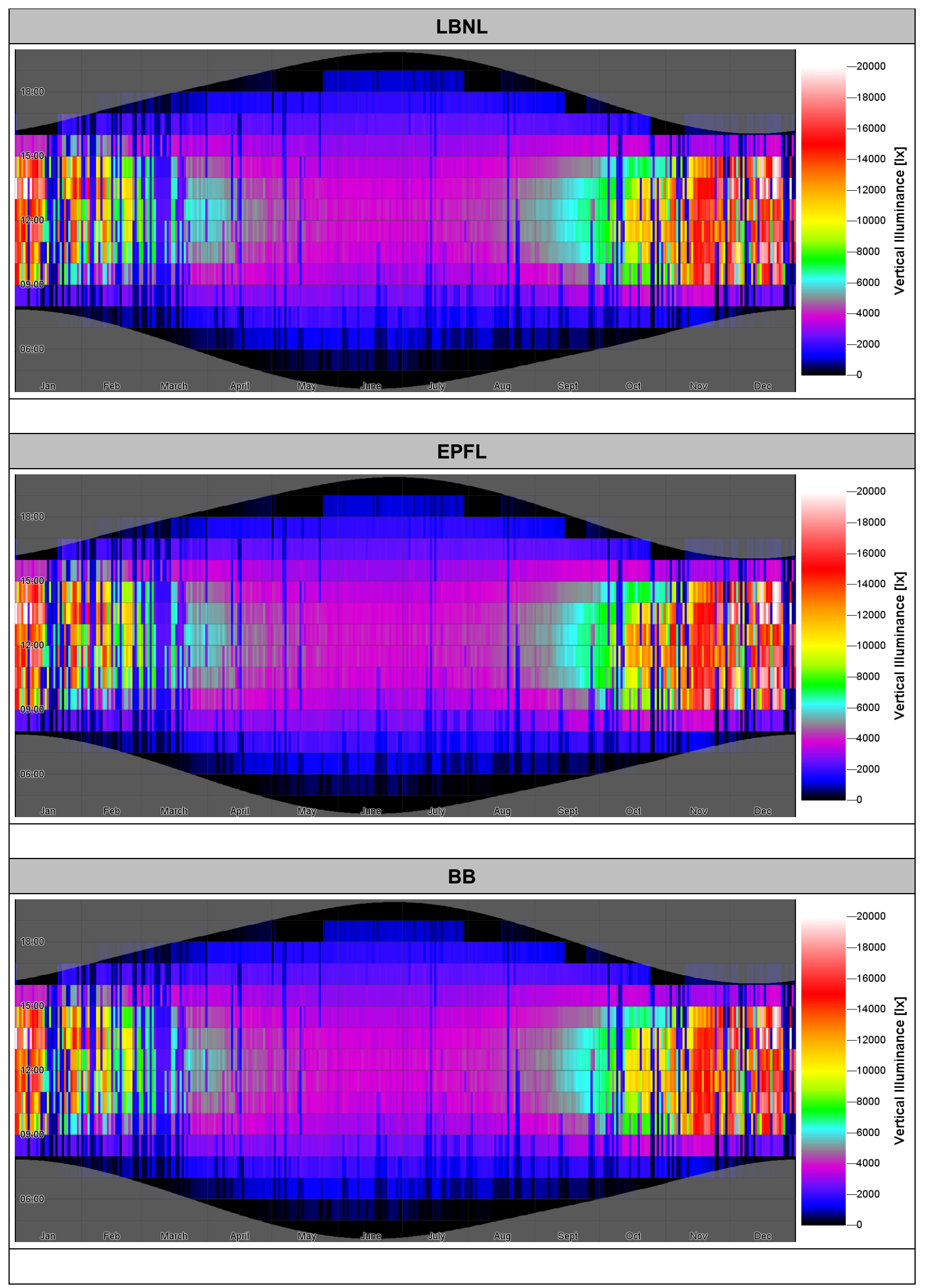

Figure 67: (2/2) Annual vertical illuminance at camera position using HELLA ARB 80 BSDF data sets in Klems resolution in 3PM calculation. 


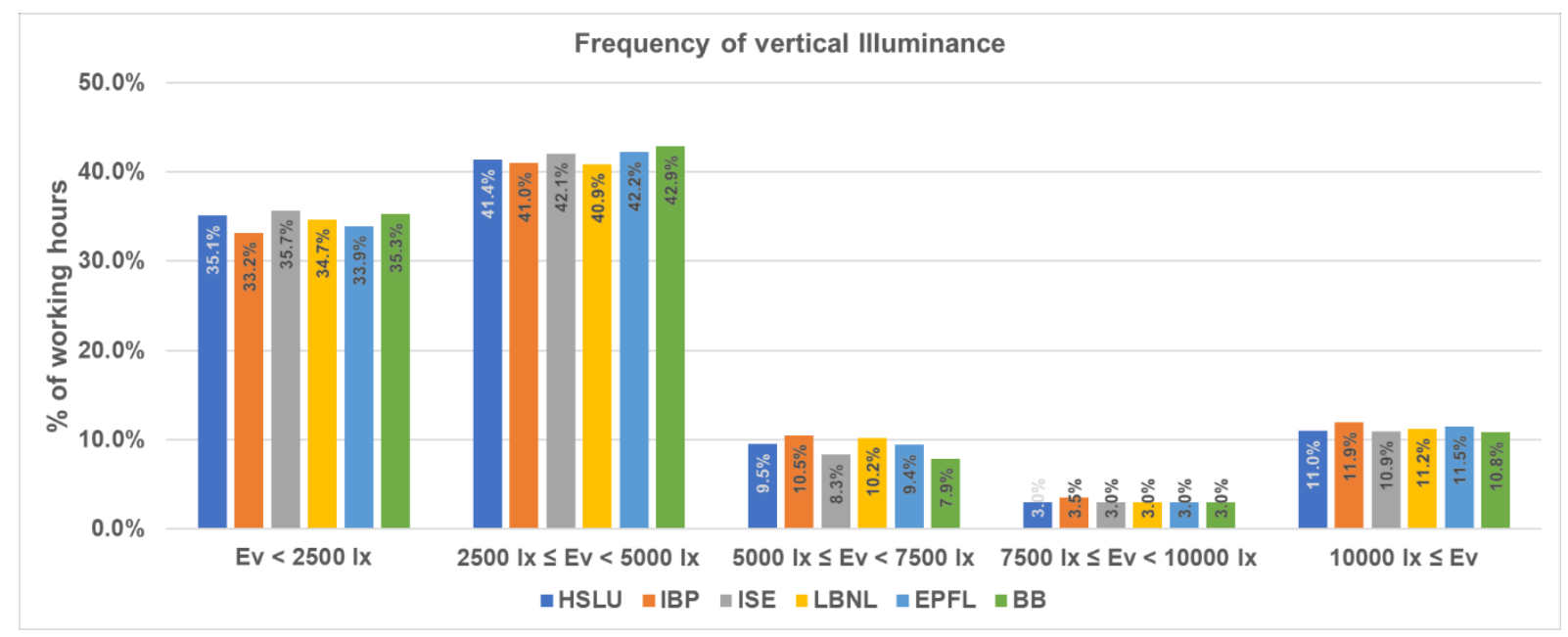

Figure 68: Occurrence during working hours (08:00-18:00) of vertical illuminance at camera position using HELLA ARB 80 BSDF data sets in Klems resolution in 3PM calculation. 


\subsubsection{Annual calculation of glare indices DGP and DGI}

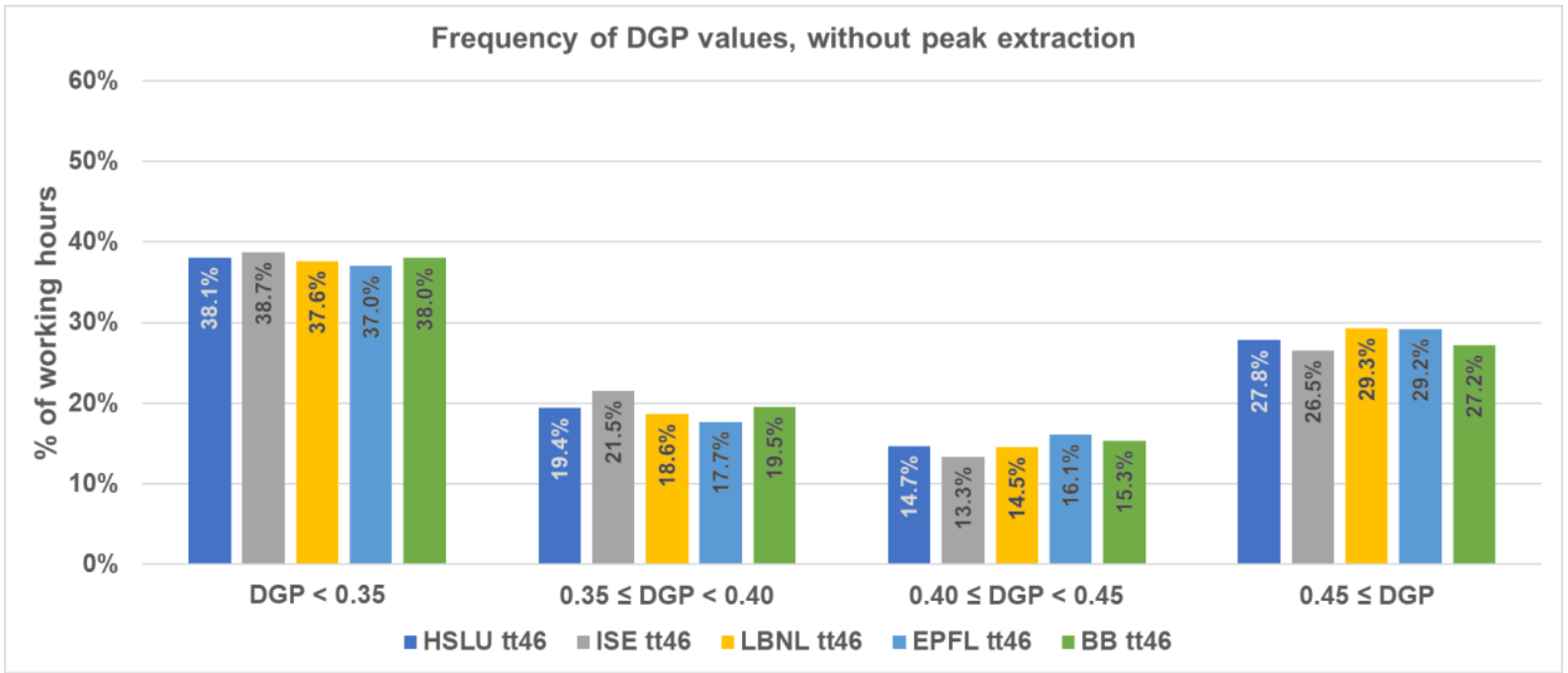

Figure 69: Frequency of DGP values during working hours (08:00-18:00) in EN17037 classes using HELLA ARB 80 BSDF data sets in high resolution (max. 4096x4096) without peak extraction ("BSDF" material model) in 5PM calculation.

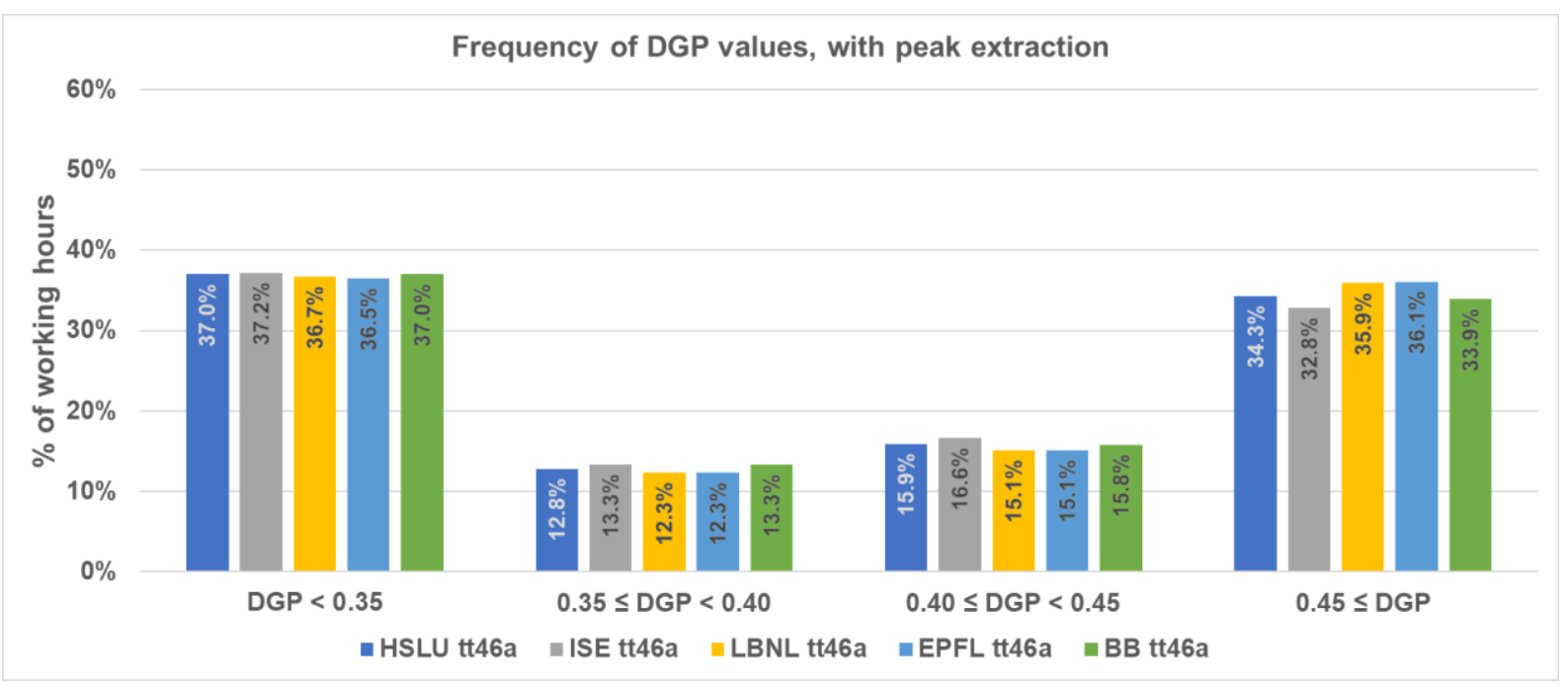

Figure 70: Frequency of DGP values during working hours (08:00-18:00) in EN17037 classes using HELLA ARB 80 BSDF data sets in high resolution (max. 4096x4096) with peak extraction ("aBSDF" material model) in 5PM calculation. 


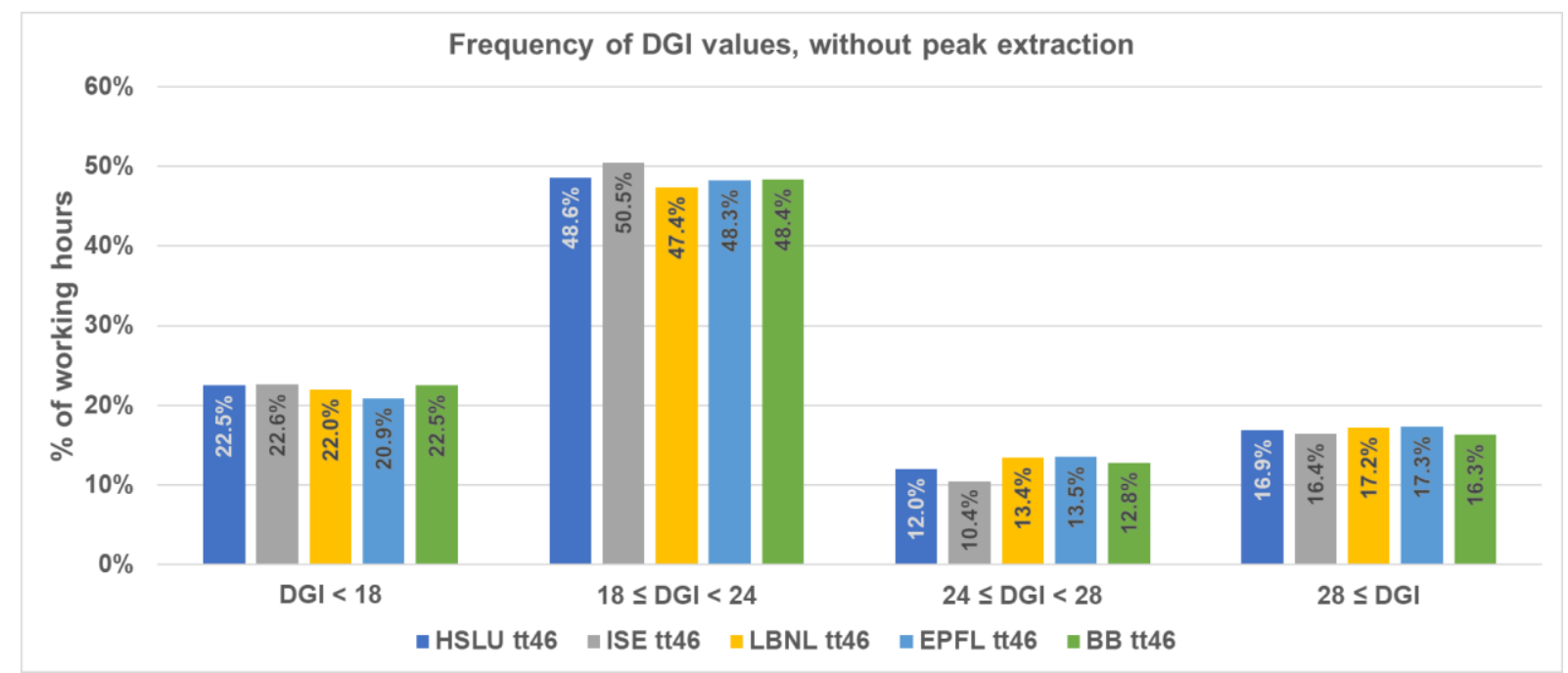

Figure 71: Frequency of DGI values during working hours (08:00-18:00) in common classes (18/24/28) using HELLA ARB 80 BSDF data sets in high resolution (max. 4096x4096) without peak extraction ("BSDF" material model) in 5PM calculation.

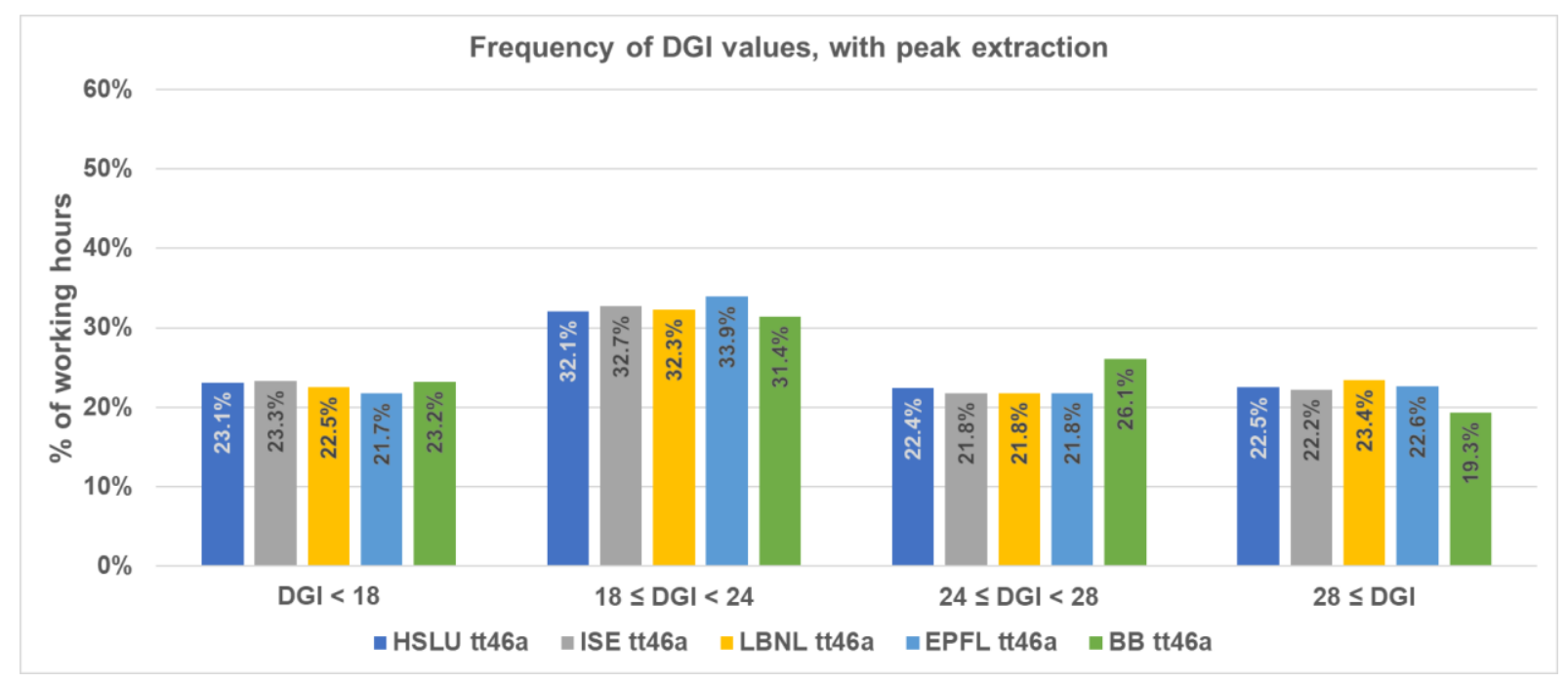

Figure 72: Frequency of DGI values during working hours (08:00-18:00) in common classes (18/24/28) using HELLA ARB 80 BSDF data sets in high resolution (max. 4096x4096) with peak extraction ("aBSDF" material model) in 5PM calculation. 


\subsubsection{MechoShade 6216}

\subsubsection{Daylight autonomy}

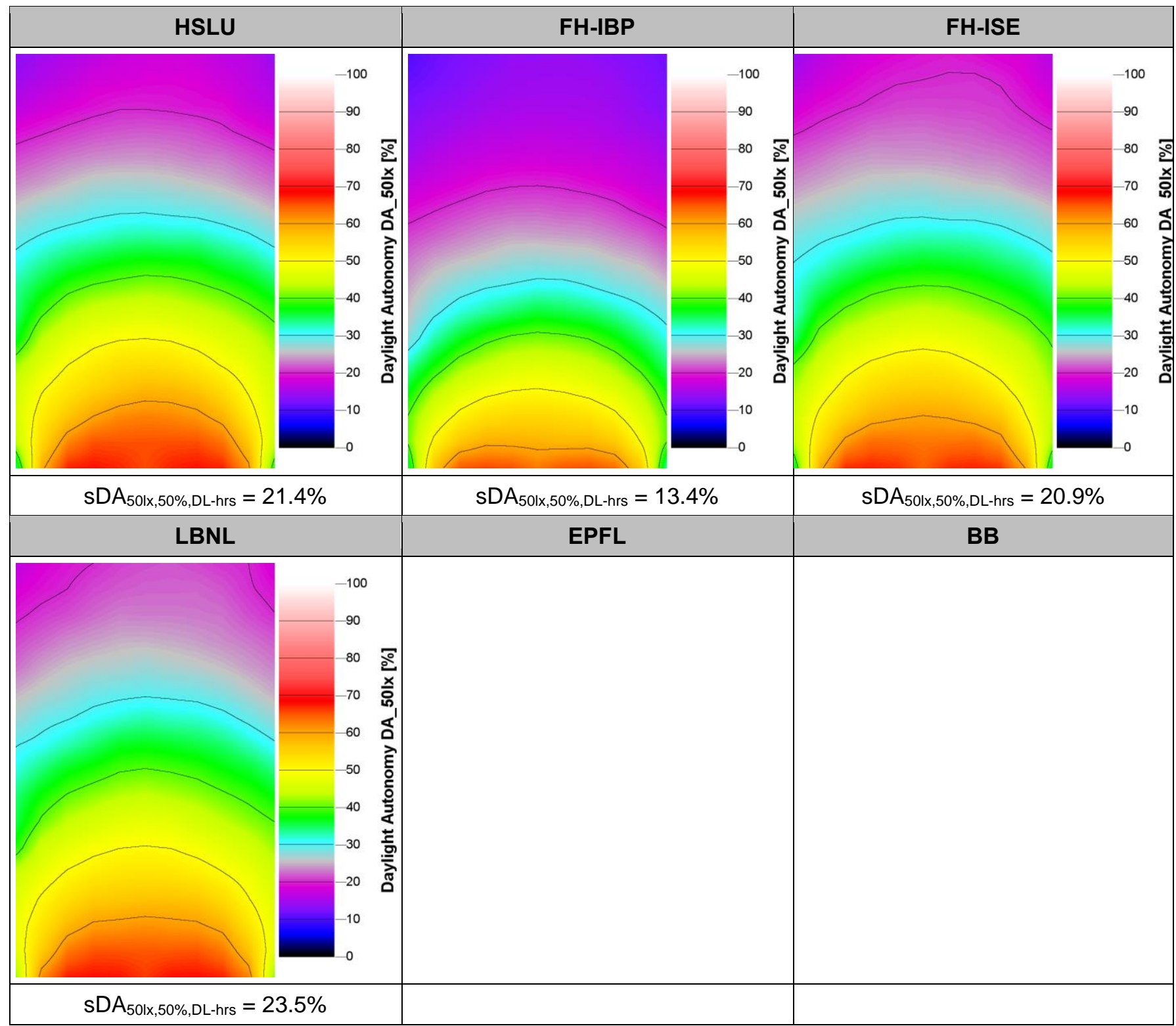

Figure 73: Daylight autonomy (50lx) based on all daylight hours on workplane using MechoShade 6216 BSDF data sets in Klems resolution in 3PM calculation. 


\subsubsection{Annual vertical illuminance at camera position}

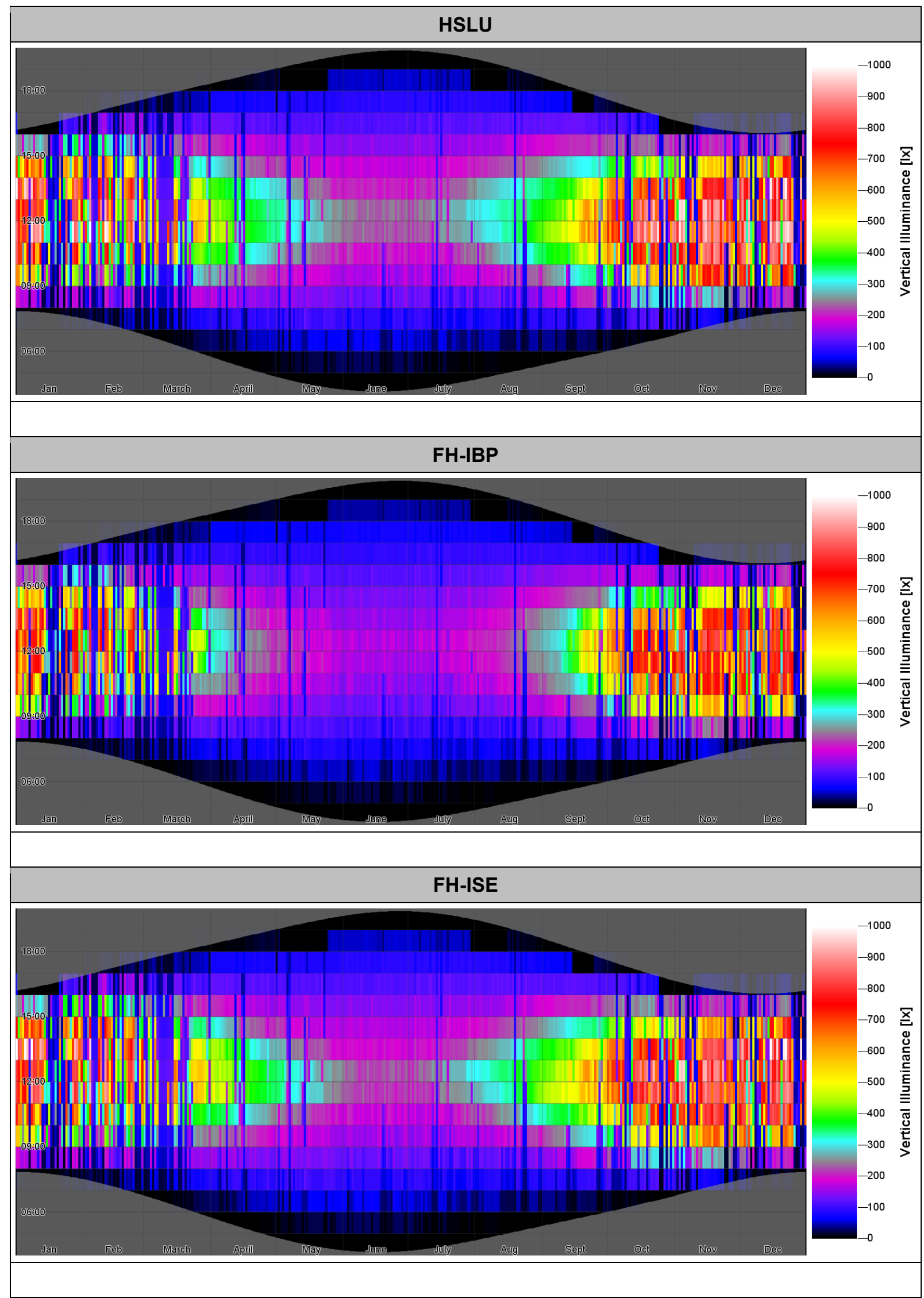

Figure 74: (1/2) Annual vertical illuminance at camera position using HELLA ARB 80 BSDF data sets in Klems resolution in 3PM calculation. 


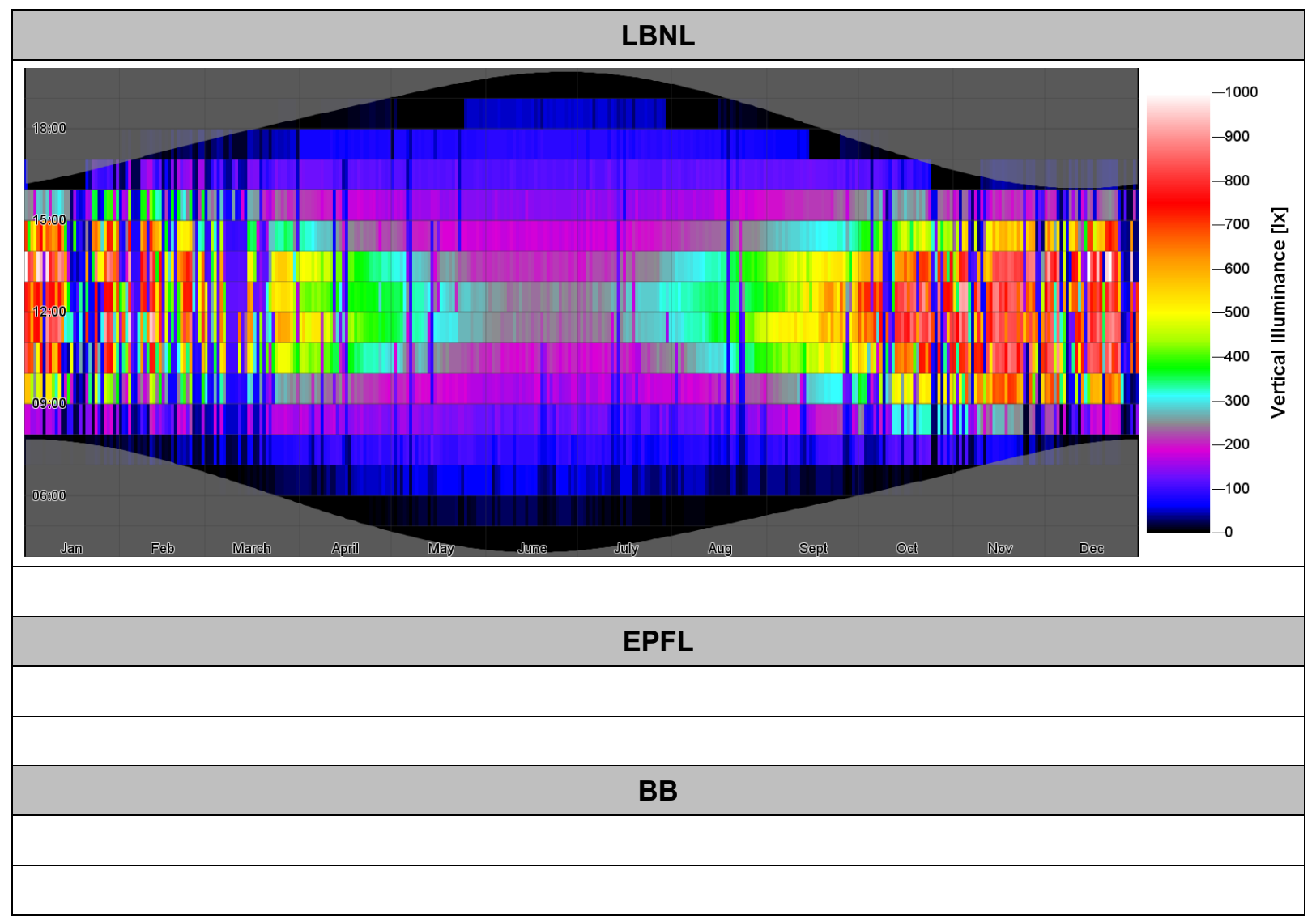

Figure 75: (2/2) Annual vertical illuminance at camera position using MechoShade 6216 BSDF data sets in Klems resolution in 3PM calculation.

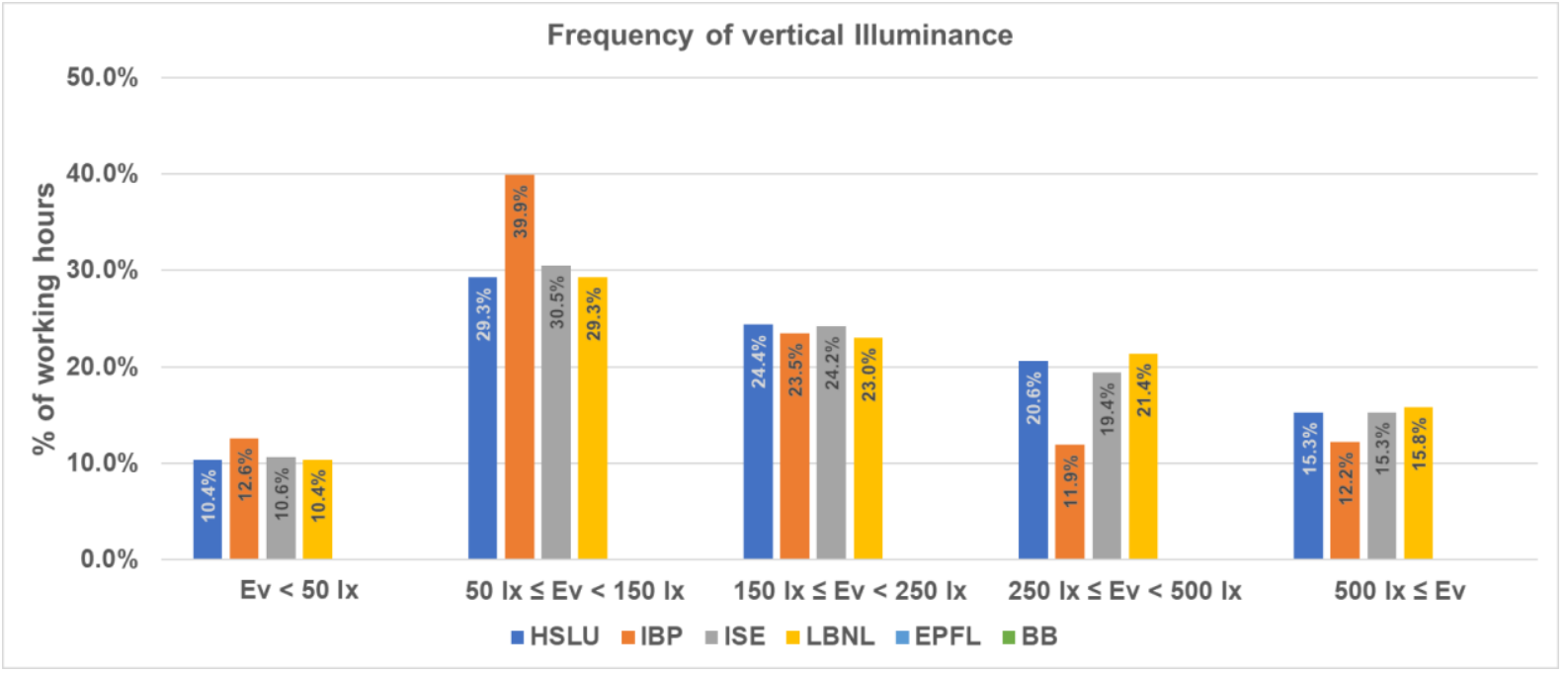

Figure 76: Occurrence during working hours (08:00-18:00) of vertical illuminance at camera position using MechoShade 6216 BSDF data sets in Klems resolution in 3PM calculation. 


\subsubsection{Annual calculation of glare indices DGP and DGI}

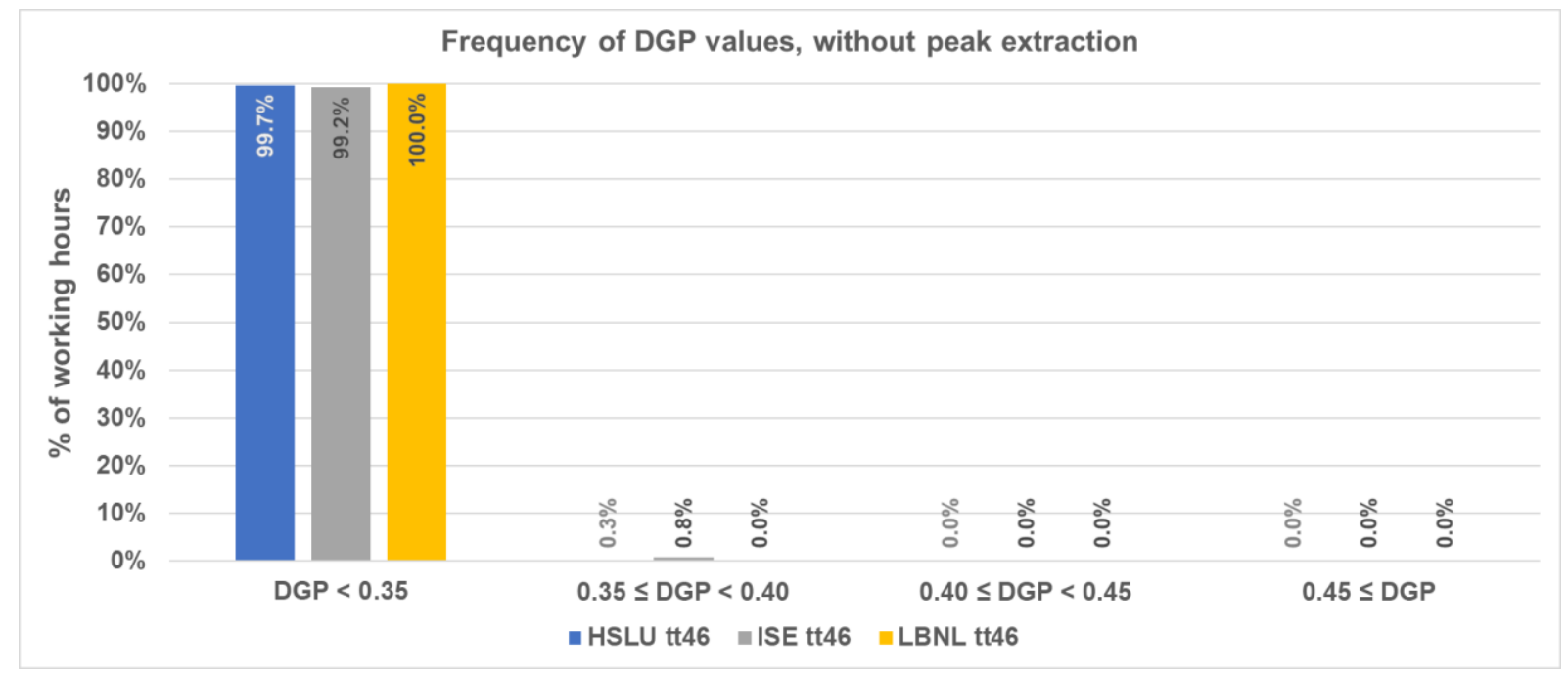

Figure 77: Frequency of DGP values during working hours (08:00-18:00) in EN17037 classes using MechoShade 6216 BSDF data sets in Klems and high resolution (max. 4096x4096) without peak extraction ("aBSDF" material model) in 5PM calculation.

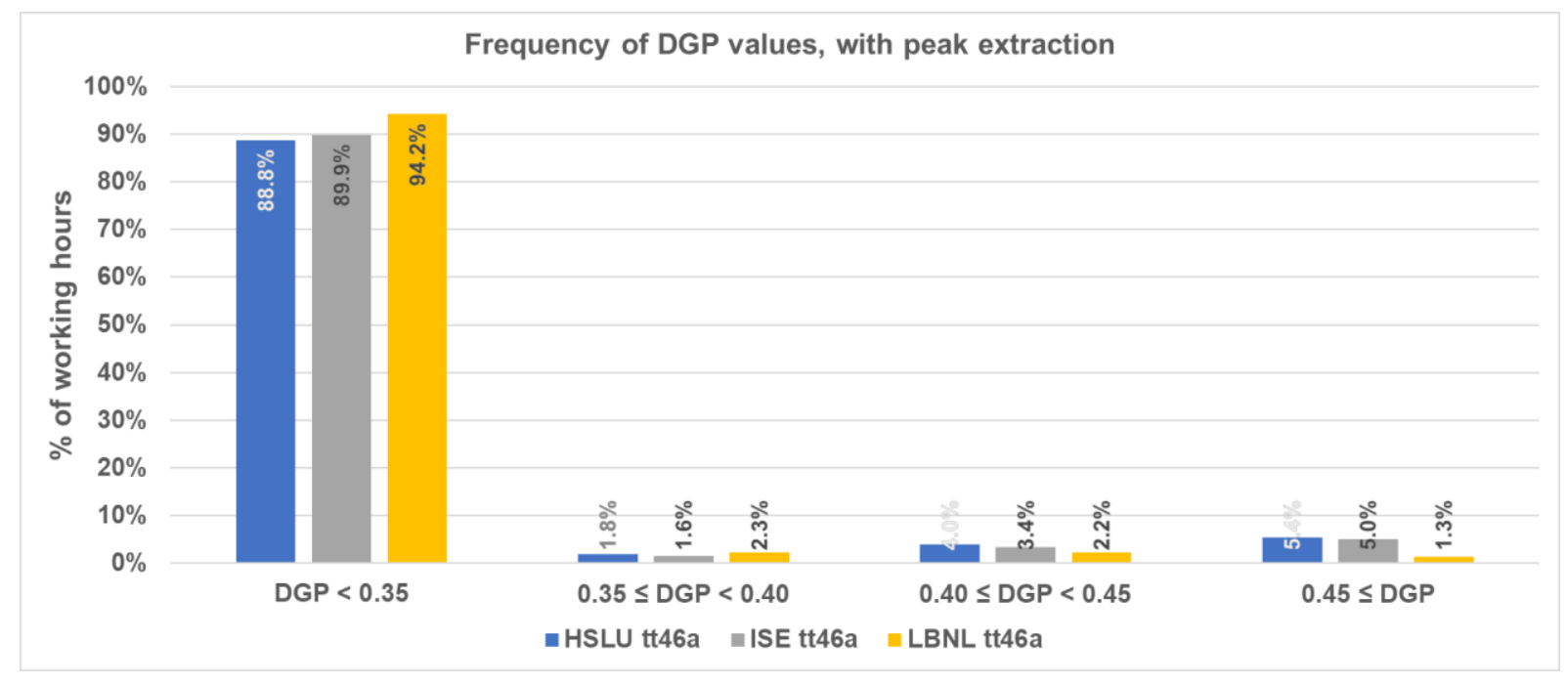

Figure 78: Frequency of DGP values during working hours (08:00-18:00) in EN17037 classes using MechoShade 6216 BSDF data sets in Klems and high resolution (max. 4096x4096) with peak extraction ("aBSDF" material model) in 5PM calculation. 


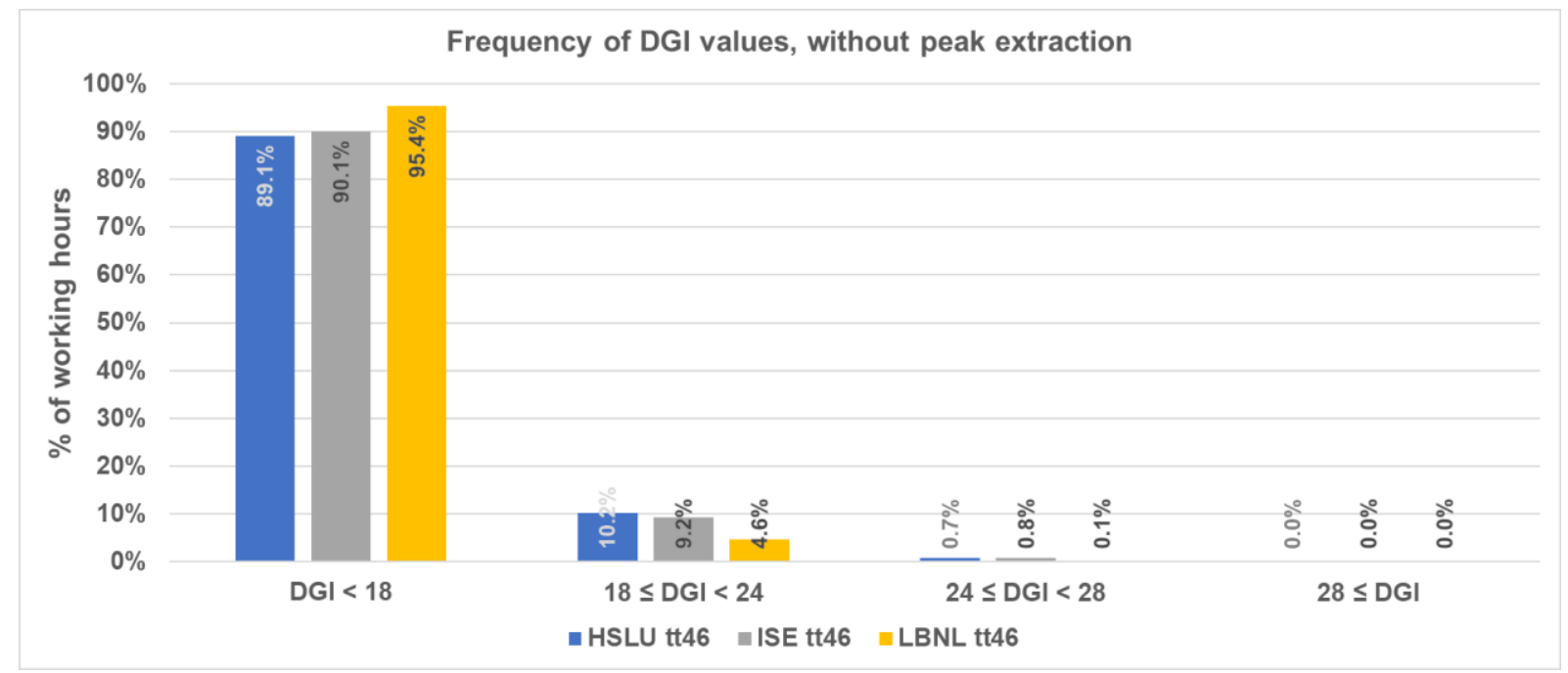

Figure 79: Frequency of DGI values during working hours (08:00-18:00) in common classes (18/24/28) using MechoShade 6216 BSDF data sets in Klems and high resolution (max. 4096x4096) without peak extraction ("BSDF" material model) in 5PM calculation.

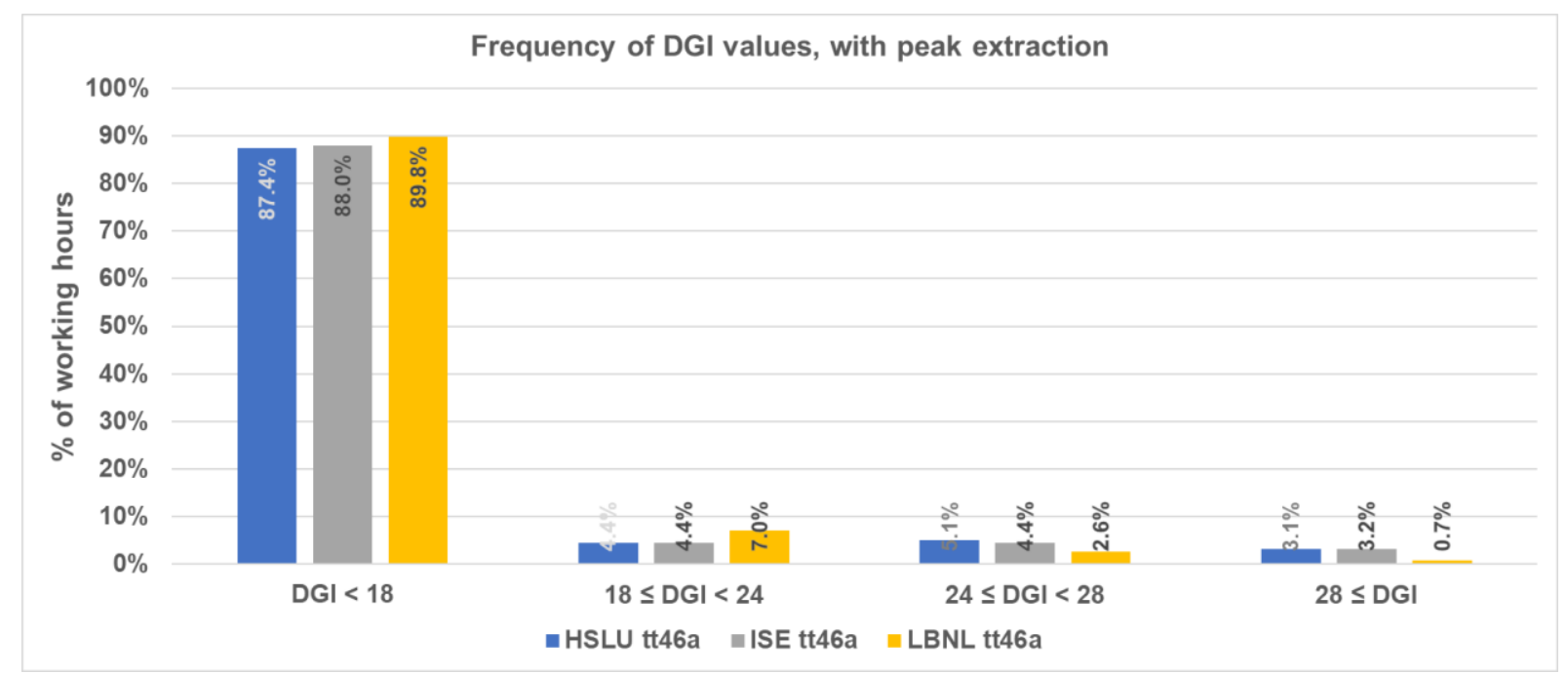

Figure 80: Frequency of DGI values during working hours (08:00-18:00) in common classes (18/24/28) using MechoShade 6216 BSDF data sets in Klems and high resolution (max. 4096x4096) with peak extraction ("aBSDF" material model) in 5PM calculation. 


\subsection{Conclusion}

It should be mentioned at the outset that all evaluations are purely fictitious and have no relation to realworld scenarios. For example, a blind system would never always remain in the horizontal position if glare protection is needed, likewise a dark glare-protection screen would never be activated all year round. Thus, the resulting evaluations from the comparative simulations are useful for comparing the underlying data sets but provide no indication of good or bad performance in the simulated rooms.

The results of the comparison show that there is good agreement between the BSDF data sets provided by the different laboratories. Keeping in mind that while both inter- and intra-institutional differences in the BSDF generation methods exist, including physical and virtual measurement instruments and setups, evaluation routines, software for data processing and validation approaches, the remaining differences are very small. This also corresponds well with earlier results ${ }^{51,52}$ and findings where remaining differences could be explained by different instrument signatures ${ }^{53}$.

On the data management side, it is positive to mention that all data was either provided in XML files ready for use in daylight simulation software, or as raw data in ASCII text files. The latter allows to use (maximum with a little reformatting) existing BSDF data processing software as for example the tools from the Radiance simulation toolbox (see section 2.10.2).

The direct evaluation of the transmittance components of the different BSDF data sets show a good match (see section 3.4.). The direct-hemispherical transmittance determines the overall light flux transmitted from a specific direction and is thus a necessary condition for correct daylighting simulations. The integrated hemispherical-hemispherical transmittance values are predicted between $47.8 \%$ and $50.3 \%$ for the HELLA blind system, and between $2.11 \%$ and $2.50 \%$ for the MechoShade fabric.

An even more important result is the agreement of predicted daylight performance metric values from daylighting simulations based on the various BSDF data sets. Especially the illuminance-based evaluations for workplane daylight autonomy and vertical illuminance evaluations show good agreement for the different underlying BSDF data sets. This was also to be expected as the illuminance as an integral value is rather good-natured towards variations in the distributions.

Generally, even with keeping all simulation parameters the same, the stochastic nature of the underlying raytracing process causes differences in the simulation results. This can be seen in particular in the deviations in the results of the point-in-time evaluations using high resolution data sets. Still, even the evaluations of luminance-based daylight performance metrics show a good agreement between the different BSDF data sets. By contrast, other calculations, in particular glare evaluations using the DGP or DGI metrics, are highly dependent on an appropriate representation of small but bright glare sources such as the sun. Here it can be seen that the selection of the simulation method ${ }^{54}$ (e.g., with or without extracting the peak from the BSDF and separately modeling the sun) has a much greater impact on the result than the deviations between the different laboratories' data sets. Current research on the high sensitivity of the DGP metric to small but bright glare sources is currently ongoing and should result in a revised formula soon ${ }^{55}$.

For more information about data-driven, tabulated BSDFs for daylighting systems, resolution schemes, approaches for their generation, and a discussion about still open questions and issues to be solved, the reader is referred to the IEA SHC Task 61's white paper on BSDF generation procedures for daylighting systems ${ }^{56}$.

51 F. Maamari, M. Andersen, J. de Boer, W.L. Carroll, D. Dumortier, P. Greenup. Experimental validation of simulation methods for bi-directional transmission properties at the daylighting performance level. Energy and Buildings, Volume 38, Issue 7, 2006, https://doi.org/10.1016/..enbuild.2006.03.008.

52 L.O. Grobe, A. Noback, S. Wittkopf, Z.T. Kazanasmaz. Comparison of measured and computed BSDF of a daylight redirecting component. CISBAT 2015, September 9-11, 2015, Lausanne, Switzerland.

53 M. Krehel, J. Kämpf, S. Wittkopf. Characterisation and Modelling of Advanced Daylight Redirection Systems with Different Goniophotometers. CISBAT 2015, September 9-11, 2015, Lausanne, Switzerland.

54 G. Ward, T. Wang, D. Geisler-Moroder, E.S. Lee, L.O. Grobe, J. Wienold, J.C. Jonsson. Modeling specular transmission of complex fenestration systems with tabulated BSDFs. Building and Environment 196, 2021.

$55 \mathrm{~J}$. Wienold, personal correspondence, 2021.

56 D. Geisler-Moroder, E.S. Lee, G. Ward, B. Bueno, L.O. Grobe, T. Wang, B. Deroisy, H.R. Wilson. BSDF generation procedures for daylighting systems. White paper. T61.C.2.1 - A Technical Report of Subtask C, IEA SHC Task 61 / EBC Annex 77. https://task61.iea-shc.org/publications. 2021. 